\title{
Structure and functionality of a multimeric human COQ7:COQ9 complex
}

3

\begin{abstract}
Mateusz Manicki ${ }^{1,2,12}$, Halil Aydin ${ }^{3,4,12}$, Luciano A. Abriata ${ }^{5,6,7}$, Katherine A. Overmyer ${ }^{2,8,9}$, Rachel M. Guerra $^{1,2}$, Joshua J. Coon ${ }^{2,8,9,10}$, Matteo Dal Peraro ${ }^{5,7}$, Adam Frost $^{3,11^{*}}$ and David J. Pagliarini ${ }^{1,2 *}$

${ }^{1}$ Department of Cell Biology and Physiology, Washington University School of Medicine, St. Louis, Missouri 63110, USA. ${ }^{2}$ Morgridge Institute for Research, Madison, WI 53715, USA. ${ }^{3}$ Department of Biochemistry and Biophysics, University of California, San Francisco, San Francisco, CA 94158, USA. ${ }^{4}$ Department of Biochemistry, University of Colorado Boulder, Boulder, CO 80309, USA. ${ }^{5}$ Institute of Bioengineering, School of Life Sciences, École Polytechnique Fédérale de Lausanne, CH-1015 Lausanne, Switzerland. ${ }^{6}$ Protein Production and Structure Core Facility, School of Life Sciences, École Polytechnique Fédérale de Lausanne, CH-1015 Lausanne, Switzerland. ${ }^{7}$ Swiss Institute of Bioinformatics, CH-1015 Lausanne, Switzerland. ${ }^{8}$ National Center for Quantitative Biology of Complex Systems, Madison, WI 53562, USA. ${ }^{9}$ Department of Biomolecular Chemistry, University of Wisconsin-Madison, Madison, WI 53562, USA. ${ }^{10}$ Department of Chemistry, University of Wisconsin-Madison, Madison, WI 53506, USA. ${ }^{11}$ Chan Zuckerberg Biohub, San Francisco, CA, USA. ${ }^{12}$ These authors contributed equally: Mateusz Manicki, Halil Aydin*e-mail: adam.frost@ucsf.edu; pagliarini@wustl.edu
\end{abstract}

\section{Abstract}

\section{Coenzyme $Q(C o Q$, ubiquinone) is a redox-active lipid essential for core metabolic}

pathways and antioxidant defense. CoQ is synthesized upon the mitochondrial inner membrane by an ill-defined 'complex Q' metabolon. Here we present a structure and functional analyses of a substrate- and NADH-bound oligomeric complex comprised of two complex Q subunits: the hydroxylase COQ7, which performs the penultimate step in CoQ biosynthesis, and the prenyl lipid-binding protein COQ9. We reveal that COQ7 adopts a modified ferritin-like fold with an extended hydrophobic access channel whose substrate binding capacity is enhanced by COQ9. Using molecular dynamics simulations, we further show that two COQ7:COQ9 heterodimers form a curved tetramer that deforms the membrane, potentially opening a pathway for $\mathrm{CoQ}$ intermediates to translocate from within the bilayer to the proteins' lipid-binding sites. Two such tetramers assemble into a soluble octamer, closed like a capsid, with lipids captured within. Together, these 
Quinones are central to most molecular energy transducing systems in organisms across all participate in electron transfer between proteins involved in diverse processes, including respiratory and photosynthetic complexes ${ }^{2,3}$. Despite their profound roles in life, many aspects of quinone biosynthesis and trafficking remained undefined ${ }^{4,5}$.

42 and that is best known for its key role in the mitochondrial electron transport chain ${ }^{7}$. CoQ comprises a redox-active head group that can carry electrons and an extremely hydrophobic isoprenoid lipid tail that drives its partitioning into the hydrophobic portion of the bilayer. In eukaryotes, the head group is derived from aromatic amino acids and imported into mitochondria where it becomes attached to the isoprenoid tail. The head group is then chemically modified by several enzymes, each of which must access intermediates that are likely buried within the membrane ${ }^{4}$. Interestingly, individual intermediates of the pathway are difficult to detect, suggesting that substrate channeling might occur between the biosynthetic enzymes (COQ proteins). Supporting this, recent work has begun to define a dynamic complex of COQ proteins

51 (complex Q, or CoQ-synthome) that may act in a metabolon-like fashion ${ }^{8-10}$. 
biochemical characterization. Protein interaction maps in Saccharomyces cerevisiae and human cells, however, suggest the complex is comprised of at least seven proteins (COQ3, COQ4, COQ5, COQ6, COQ7, COQ8, COQ9) ${ }^{11-13}$. The complex architecture and subunit stoichiometry remain unknown, as does whether discrete sub-complexes exist. However, observations in yeast $^{14}$, mice ${ }^{15}$, and humans ${ }^{16}$ have shown that a direct physical and functional relationship exists between COQ7, an iron-dependent hydroxylase, and COQ9, a lipid-binding auxiliary protein, and that mutations in either protein lead to the accumulation of the penultimate CoQ intermediate, demethoxy-coenzyme Q (DMQ). Mutations of these proteins result in neurological defects, decreased activity of the mitochondrial electron transport chain, reduced levels of ATP synthesis, and increased mitochondrial oxidative stress ${ }^{16-23}$. membrane association and/or in accessing a CoQ intermediate. However, it is still unclear how COQ proteins surmount hydrophobic barriers to access CoQ intermediates, which reside entirely within the bilayer. To explore these ideas, we co-expressed and co-purified an octameric COQ7:COQ9 complex and solved its atomic structure using cryoEM. The structures show that

71 COQ7 binds COQ9 via an extended ferritin-like fold, binds a CoQ precursor, and binds its

72 NADH cofactor along with additional lipids. Within this complex, COQ9—which, on its own,

73 binds CoQ precursors robustly - is in the apo form, suggesting that it may have delivered this

74 ligand to COQ7. The octameric complex consists of two COQ7:COQ9 tetramers held together

75 through their hydrophobic faces by various small hydrophobic molecules. While the significance

76 of the octameric state of the complex remains unclear, biochemistry and simulations suggest that 
77 the tetrameric halves of the octamer may bind and bend target membranes, to enable these

78 proteins to access lipids within the bilayer. Together, our data provide an initial insight into how

79 a metabolon-like CoQ complex could assist the biosynthesis of a hydrophobic molecule at the

80 membrane.

81

\section{Results}

\section{CryoEM structure of human COQ7}

84 COQ7 is a membrane-bound di-iron protein and an enigmatic member of the ferritin family that

85 has been recalcitrant to experimental structural characterization ${ }^{25-27}$. Here, using an E. coli

86 expression system, we co-expressed human COQ7 (residues 39-217 with N-terminal GB1 tag ${ }^{28}$ )

87 together with its native partner, human COQ9 (residues 79-318 with an N-terminal His tag), and

88 performed metal affinity and gel filtration chromatography. Our results revealed that COQ7 and

89 COQ9 co-purify as a 1:1 complex with a molecular weight of $\sim 240 \mathrm{kDa}$, suggesting a likely

90 octamer (Fig. 1a and Extended Data Fig. 1a-d). Further biophysical characterization by negative-

91 stain electron microscopy confirmed the homogeneity of the sample and revealed monodispersed

92 particles of the intact COQ7:COQ9 complexes with symmetric features (Extended Data Fig.

$931 \mathrm{e}, \mathrm{f})$.

The complex was vitrified, imaged, and reconstructed using 3D single-particle cryoEM

95 analysis. An initial density map reached $\sim 3.5 \AA$ but was incompletely occupied by the known

97 COQ7:COQ9 hetero-octamers with excess NADH prior to vitrification. This second dataset

98 yielded an improved map at an overall nominal resolution of $\sim 2.4 \AA$, with D2 symmetry, and

99 robust density for NADH (Extended Data Fig. 4-5, Extended Data Table 1). Most of the side 
chains were well-resolved and allowed de novo atomic model building of COQ7 and COQ9, with the exception of the flexible N-terminal GB1 tag on COQ7 and C-terminal $10^{\text {th }} \alpha$-helix of COQ9 (Extended Data Fig. 6, Extended Data Table 1).

COQ7 has a rod-shaped structure (Fig. 1b) with approximate dimensions of $45 \AA$ X $30 \AA$ X $27 \AA$ and shares structural similarity with bacterioferritin $(\sim 1.7 \AA$ r.m.s.d. over $173 \mathrm{C} \alpha$ atoms of COQ7) (Extended Data. Fig. 7a). However, major structural changes are apparent and suggest that the fold was repurposed from the classic iron-storing ferritin to a membrane-binding hydroxylase (Extended Data Fig. 7b). The overall structure is comprised of six $\alpha$-helices $(\alpha 1-\alpha 6)$ (Fig. 1b). Helices $\alpha 1, \alpha 2, \alpha 4$, and $\alpha 5$ exhibit a compact four-helix bundle containing the di-iron center, which is able to bind two metal ions ( $\mathrm{Fe} 1$ and $\mathrm{Fe} 2)$. The three-dimensional map of COQ7 presents the atomic coordinates of the structurally and functionally important iron coordination motif $\left(E-\left(X_{6}\right)-Y-\left(X_{22}\right)-E-\left(X_{2}\right)-H-\left(X_{48}\right)-E-\left(X_{6}\right)-Y-\left(X_{28}\right)-E-\left(X_{2}\right)-H\right)$, which is conserved across diiron carboxylate proteins, supporting the enzymatic function of the protein as a hydroxylase (Extended Data Fig. 7c). While the protein chains were well defined by electron density, we did not observe electron density for the active site ions and could only estimate their position. By analogy with bacterioferritin, the first metal ion binding site, Fe1, would be coordinated by E60, H93, Y149, and E178, whereas the Y67, E90, E142, and H181 residues would coordinate the Fe2 site.

In addition to the four-helix bundle, COQ7 structure contains a short helix ( $\alpha 3)$ and a Cterminal helix ( $\alpha 6)$ that is connected to the rest of the molecule via a long loop. These features represent a novel organization for ferritin-like di-iron carboxylate enzymes (Extended Data Fig. 7d). The helices $\alpha 3$ and $\alpha 6$ contribute to the formation of a large hydrophobic channel along with $\alpha 1$ and $\alpha 4$ (Fig. $1 \mathrm{~b}$ and Extended Data Fig. 7e), and a flat hydrophobic surface on the membrane- 
123 proximal side of the protein (Fig. 1c). The residues L111, L114, L118, L122, I202, V209, L213,

124 and L217 define the outer entrance to the channel, whereas the inner surface is lined by A63, I66,

125 W115, V141, I145, I203, C207, A210, and I211. This hydrophobic surface may interact with the

126 surface of the mitochondrial inner membrane, and the channel is sufficiently large to admit the

127 entry of quinone intermediates and access to the di-iron center. A similar structural feature can

128 be found in alternative oxidases (AOX) that also belong to the ferritin family and that use CoQ

129 as a substrate for different roles in the cell ${ }^{29}$ (Extended Data. Fig. 7f-h).

We also observed a clearly defined isoprenoid quinone-like density extending from the inner surface of the hydrophobic channel and making several contacts with the hydrophobic surface (Fig. 1d and Extended Data Fig. 6d). The quinone head group fits comfortably into the

133 hydrophobic pocket and the isoprene tail adopts a U-shaped conformation that is stabilized by 134 conserved hydrophobic residues (L118, A121, L122, L129, L199, and I202) located on the 135 protein surface. To determine the identity of this isoprenoid, we performed liquid chromatography with tandem mass spectrometry (LC-MS/MS) of the purified COQ7:COQ9

137 complex and of bacterial cells used for its production. The protein sample showed 50x

138 enrichment ( $20 \%$ of total lipid signal) in octaprenylphenol (OPP), a bacterial CoQ biosynthetic

139 intermediate $^{30}$ (Fig. 1e). In agreement with the logic of biosynthetic pathways, no enrichment of

$140 \mathrm{CoQ}_{8}$ (the E. coli version of CoQ) was detected showing specificity of the proteins towards the

141 CoQ intermediate and exclusion of the mature CoQ product (Fig. 1f). Together, these results

142 identify OPP as the bacterial ligand occupying the hydrophobic channel leading to the active site 143 of COQ7.

To functionally validate these observations, we turned to yeast that can survive without

$145 \mathrm{CoQ}$ in fermentation but require it for respiration. Structural features and evolutionary 
conservation of COQ7 (Extended Data Fig. 8a) guided us in the design of mutations that should block the hydrophobic channel. As expected, mutations G123Y, M127W, I219Y, I222Y, or A226Y (corresponding to human L118, L122, I203, G206, or A210 residue) that introduce a large hydrophobic residue into the channel, reduced CoQ 6 levels in yeast and their ability to respire (Fig. 1g, h) without affecting the proteins' stability as judged by their expression levels (Fig. 1i). To further confirm specific disruption of Coq7's function in the yeast mutants we used mass-spectrometry to measure levels of $\mathrm{DMQ}_{6}$, the bona fide substrate of Coq7. In all cases, we detected the molecule showing that upstream steps of $\mathrm{CoQ}_{6}$ biosynthesis are at least partially functional and that low Coq7 activity may constitute a pathway bottleneck in the mutant strains (Fig. 1j). Together, these data strongly support in vivo existence of the hydrophobic channel, as evidenced by the COQ7 structural model. Of note, the structure helps to understand the deleterious effects of COQ7 mutations found in human patients, including a recent case of K200fs56 mutation ${ }^{17}$ that localizes to COQ7's $6^{\text {th }}$ helix revealed for the first time in this manuscript (Extended Data Fig. 8b).

\section{CryoEM reveals details of NADH binding by COQ7}

To achieve their enzymatic activity, di-iron proteins require a reduction of the iron atoms in their active site from $\mathrm{Fe}^{3+}$ to $\mathrm{Fe}^{2+}$ state $^{31}$. It has been shown in vitro that quinone substrates can mediate the transfer of electrons from NADH to COQ7's active site ${ }^{32}$. Comparison of our unsoaked and NADH-soaked reconstructions showed how NADH binds to COQ7 (Fig. 2a) via a set of evolutionarily conserved surface-exposed residues (Extended Data Fig. 8a) without inducing global conformational changes. Although it is unknown how the charge is transferred from the NADH to the protein, it has been suggested that it might follow an uncommon, 
169

170

171

172

173

174

175

176

177

178

179

180

181

182

183

184

185

186

187

188

189

190

191

sequential electron-proton-electron donation mechanism ${ }^{26}$, and involve an amino acid triad $\mathrm{E} 60 / \mathrm{H} 148 / \mathrm{Y} 149^{32}$. In our structure the cofactor localizes next to a water-filled channel formed by the triad that might facilitate proton transfer. It is also close enough to the active site pocket $(\sim 15 \AA)$ to permit direct tunneling of electrons to the expected quinone acceptor ${ }^{33-36}$ (Fig. 2 b,c and Extended Data Fig. 9a-d,i). This biologically useful positioning of NADH is provided by the R51, R208, Y212, and R216 residues of COQ7 that contact different parts of the NADH molecule (Fig. 2d). Interestingly, the interaction between R208 and NADH is bridged by the headgroup of a clearly visible, but not identified, phospholipid molecule (Fig. 2d). This suggests a functional role of membrane lipids in the stabilization of NADH contacts.

To test the functional importance of the identified residues, we purified COQ7 alongside a quadruple COQ7 ${ }^{\mathrm{R} 51 \mathrm{~A} / \mathrm{R} 208 \mathrm{~A} / \mathrm{Y} 212 \mathrm{~A} / \mathrm{R} 216 \mathrm{~A}}$ mutant (Extended Data Fig. 9e) and tested their activity in vitro by measuring the loss of NADH fluorescence upon its oxidation to NAD + . In agreement with its proposed mechanism of action, wild type COQ7 slowly oxidized NADH and transferred electrons to a quinone acceptor, changing its redox state as measured by an HPLC equipped with a redox-sensitive electrochemical detector (HPLC-ECD) (Extended Data Fig. 9f). However, the mutant markedly lost activity (Fig. 2e,f and Extended Data Fig. 9g,h). Although arginines have been reported to play a part in NADH binding (e.g., Rex ${ }^{37}$, p-Hydroxybenzoate Hydroxylase ${ }^{38}$, Malic Enzyme ${ }^{39}$ ) they are not the most common residues found in NADH-binding motifs ${ }^{40,41}$. Therefore, we again sought to validate the biological importance of our observations in vivo using the yeast respiration system. High overexpression of $\operatorname{Coq} 7^{\mathrm{R} 54 \mathrm{~A}}$ or $\operatorname{Coq} 7^{\mathrm{R} 224 \mathrm{~A}}$ point mutants (corresponding to residues R51 and R208 in human COQ7, respectively) was not able to fully support $\mathrm{CoQ}_{6}$ production or respiratory growth. Interestingly, mutants W2228A and R232A (Y212 and R216 in humans) showed only a minor reduction in CoQ6 level, and hence fully 
192

193

194

195

196

197

198

199

200

201

202

203

204

205

206

207

208

209

210

211

212

213

214

supported respiration (Fig. 2g-i). The partial loss of the in vitro activity of the quadruple human COQ7 mutant and the reduced levels of $\mathrm{CoQ}_{6}$ in yeast mutants indicate that the NADH's positioning observed in cryoEM is biologically relevant. Furthermore, as no structure of COQ7 has yet been reported, our work provides a first glimpse into how this protein leverages its fold to perform the penultimate step of $\mathrm{CoQ}$ biosynthesis.

\section{CryoEM reveals lipid molecules bound to the COQ7:COQ9 heterodimer.}

Efficient interaction of COQ7 with COQ9 is known to be essential for CoQ production in vivo.

We previously described the crystal structure and lipid-binding activity of human COQ9 and used molecular dynamics simulations to predict its interaction with a COQ7 homology model on a lipid membrane ${ }^{24}$. Here, using cryoEM, we experimentally determined the structure of the complex bound to lipids. The atomic structure of human COQ9 ( $\alpha 1$ to $\alpha 9)$ fits well as a rigid body to the electron density of the COQ7:COQ9 complex. Interestingly, the $\alpha 7-\alpha 8$ loop adopts only one of the two conformations found in the isoprene-bound crystal structure of COQ9 (PDB:6DEW). The overall architecture of the COQ7:COQ9 heterodimer is shown in Fig. 3a. We observed an interface that is stabilized by several hydrogen bonds, hydrophobic interactions, and an intermolecular salt bridge between COQ9 (D225, D226, T236, D237, F238, W240, and Y241) and COQ7 (N98, M101, V102, R105, R107, P108, T109, V110, and M112) residues, and that spans a buried solvent-accessible surface area of approximately $468 \AA^{2}$ (Fig. 3b). Notably, this limited contact interface between COQ7 and COQ9 may not be sufficient for stable complex formation. In fact, a single point mutation in that region $\mathrm{COQ} 9^{\mathrm{W} 240 \mathrm{~K}}$ led to total disruption of the octameric $240 \mathrm{kDa}$ COQ7:COQ9 complex and formation of a smaller $\sim 55 \mathrm{kDa}$ complex comprised only of COQ9 (Fig. 3c and Extended Data Fig. 10a-c). This shows that W240 is 
crucial for this contact site between $\mathrm{COQ} 7$ and $\mathrm{COQ} 9$, which ultimately leads to formation of a higher mass complex stabilized by lipids, isoprenes and NADH.

In particular, investigation of the $2.4 \AA$ cryoEM density map revealed multiple lipid-like densities engaged with the surface of the proteins. They were not randomly oriented, but rather formed a $30 \AA$ tall, bilayer-like and warped sheet with lipid tails pointing towards each other and the headgroups pointing in opposite directions (Fig. 3d). The lipids likely originate from E. coli plasma membrane based on our lipidomics results ${ }^{42,43}$ (Extended Data Fig. 10d). These observations may have biological relevance because quinones are known to be trapped within the membranes. Due to their extreme hydrophobicity, these molecules cannot transverse through the headgroup layer ${ }^{44-49}$ and thus likely have to be extracted by proteins.

We previously determined the crystal structure of COQ9 with a hydrophobic isoprenoid substrate and identified a lipid-binding pocket inside the protein. In the cryoEM structure, this pocket is located exactly between the displaced lipid headgroups, consistent with a model in which COQ9's binding reorganizes the membrane to enable access to its interior. Interestingly, we did not observe a well-defined electron density for lipids resembling CoQ intermediates within the lipid-binding cavity of COQ9 (Fig. 3f) which could indicate that the quinone molecule was transferred to COQ7. If that is the case, then COQ7 should be enriched in OPP when in complex with COQ9. To verify this, we measured quinone content in COQ7 and COQ9 purified alone and as a complex. As expected, the lipidomic analysis confirmed a higher OPP level in the COQ7:COQ9 complex than in COQ7 or COQ9 alone (Fig. 3g and Extended Data Fig. 10e). Knowing there is no observable OPP bound to COQ9 in the COQ7:COQ9 complex, the higher OPP signal most likely represents an increased level of OPP bound to COQ7 due to COQ9's actions. Together, our structural model reinforces and advances our previous in vitro analyses, 
and provides a detailed blueprint for defining COQ7-COQ9-lipid interactions critical for complex functionality.

\section{COQ7 and COQ9 form a heterotetramer}

It has been reported that in solubilized human mitochondria COQ7 is predominantly detected as a complex of 100-150 kDa mass ${ }^{50}$. A separate study found COQ9 to migrate in 50-150 kDa range $^{51}$. This suggests that a subcomplex of our $\sim 240 \mathrm{kDa}$ human COQ7:COQ9 octamer may represent its dominant in vivo state. In the cryoEM structure, two heterodimers of COQ7:COQ9 come together on the same side of the lipid pseudo-bilayer and form a curved tetramer with a hydrophobic surface suitable for membrane interaction. Viewed from the membrane surface, one heterotetrameric assembly is approximately $100 \AA$ in diameter and $40 \AA$ axially with a solventexposed central pore that accommodates the NADH molecule (Fig. 4a). It is formed by two COQ7:COQ9 heterodimers that make additional contacts with an interface that buries around $261 \AA^{2}$ of surface area. The N-terminal segment of the $\alpha 7$ helix region of COQ9 and $\alpha 6$ helix of COQ7 from opposing heterodimers associate around a 2-fold axis normal to the membrane plane, with most intimate contacts occurring between COQ9 residues L209, P210, and H211 and COQ7 residues S201, Q204, A205, and R208 (Fig. 4b).

To test the importance of this region, we generated corresponding yeast mutants and measured their CoQ6 levels and ability to respire. We observed that mutations of P210 and S201 - represented by $\operatorname{Coq} 9^{\mathrm{P} 155 \mathrm{R}}$ and $\operatorname{Coq} 7^{\mathrm{E} 217 \mathrm{R}}$ in yeast— led to impaired respiration and diminished $\mathrm{CoQ}_{6}$ levels, despite high expression levels of the mutated proteins (Fig. 4c-e). Over time, yeast did display moderate respiratory growth showing that the proteins remain at least partially functional. Given the magnitude of the Coq9 $9^{\mathrm{P} 155 \mathrm{R}}$ phenotype, we queried whether 
261 disruption of that residue disturbs the human COQ7:COQ9 complex. The COQ9 ${ }^{\mathrm{P} 210 \mathrm{~K}}$ mutation

262 did not prevent the formation of the $240 \mathrm{kDa}$ complex expressed in bacteria at $20^{\circ} \mathrm{C}$ (Extended

263 Data Fig. 10f) suggesting that the interaction site is not fully disrupted or that protein-protein

264 interactions are not the sole contributors to its stability. Due to the large hydrophobic surfaces

265 present in COQ7 and COQ9, we hypothesize that membrane association may play an important

266 role in that stabilization.

267

268 Molecular dynamics simulations of the tetramer engaging and shaping a membrane

269 To explore whether and how the tetrameric form binds the lipid bilayer to potentially facilitate

270 transformation of the embedded quinone substrate, we performed atomistic molecular dynamics

271 (MD) simulations on systems set up with the COQ7:COQ9 heterotetramer manually placed with

272 its hydrophobic surface facing but not binding a model mitochondrial membrane. The systems

273 were parametrized as described in methods. Of note, since no parameterized models of the

274 quinone detected in the cryoEM structure (OPP) or the true COQ7:COQ9 substrate (DMQ)

275 are available, we used CoQ10 by Galassi et al. ${ }^{52}$ as an approximation of the small molecules

276 involved in COQ7 and COQ9 activities. CoQ10 was either added to the membrane at $\sim 4 \%$

277 concentration in simulations designed to test how the substrate-free protein binds to membranes,

278 or placed individually inside COQ7's active site in simulations aimed at testing its stability when

279 bound to COQ7's active site.

280 In all simulations, the tetrameric arrangement inferred from the cryoEM structure

281 interacted rapidly $(<50 \mathrm{~ns})$ with the membrane. This interaction remained stable for the whole

282 simulation (Fig. 5a). Upon this interaction, COQ7 progressively gained direct access to the

283 hydrophobic portion of the membrane (plotted as contacts between COQ7 and the carbon tails of 
284 the lipids over time in Figure 5a). As COQ7's access to the membrane proceeded, lipids

285 tilted away from a normal bilayer architecture until they resembled the angular orientations of

286 the lipids resolved in the cryoEM density map. In addition, the lipids concentrated their

287 headgroups on the periphery of the protein-membrane contact region to form a rim around the

288 membrane-facing surface of the COQ7 proteins (Fig. 5b). This arrangement exposed the carbon-

289 tail region of membrane lipids directly to the COQ7 proteins (Fig. 5b), while leaving COQ9 to

290 interact peripherally with the headgroup phosphates. As a consequence, a strong local

291 deformation of the membrane of around $10 \AA$ was observed (Fig. 5c and Extended Data Fig.

$29211 \mathrm{a}, \mathrm{b})$.

Given the direct contact between the hydrophobic surfaces of COQ7 and the internal,

294 hydrophobic portion of the membrane, we speculate that the local membrane reshaping induced

295 by the tetramer might help to facilitate the exchange of substrates and products directly from the

296 membrane into and out of the tetrameric complex. Indeed, in the simulations with model

297 membranes doped with 4\% CoQ10 we observed transient association of the small molecule with

298 the hydrophobic surface of COQ7 (Extended Data Fig. 11c). However, the aromatic headgroup

299 of CoQ10, which has similar size to COQ7's bona fide substrate DMQ, seemed too bulky to

300 access the protein's active site (Extended Data Fig. 11d). Therefore, its entry may require a

301 conformational change of COQ7 which is unlikely to spontaneously take place in microsecond-

302 long atomistic simulations.

303 To explore this idea, we devised a reversed computational experiment in which we

304 modeled a molecule of CoQ10 inside COQ7's active site based on the position of OPP molecule

305 observed in the cryoEM structure, and then monitored its release from the active site through

306 simulations. Replica-exchange simulations were impossible to tune, as they resulted in 
307

308

309

310

311

312

313

314

315

316

317

318

319

320

321

322

323

324

325

326

327

328

329

membrane disruption; we therefore tested regular simulations at various stable temperatures. At $450 \mathrm{~K}$ we observed events in which the docked CoQ10 dissociated from the active site without compromising the protein or membrane stabilities within the simulated timescale $(\sim 1-1.5$ microsecond).

Out of 10 replicas at $450 \mathrm{~K}$, we observed CoQ10 leaving the active site in two directions: 8 times directly towards and into the membrane bulk, and twice towards COQ9 before moving to the membrane bulk (Fig. 5d and Extended Data Fig. 11e). In every case, release of the ligand involved a displacement of the two COQ7 helices $(\alpha 3, \alpha 6)$ that form the hydrophobic channel leading to the active site, temporarily widening its opening towards the membrane.

Although it is hard to tell how much of this is due to the high temperatures used to promote unbinding and how much reflects realistic motions, we noticed that the residue W115 flips its sidechain a few times (Fig. 5e) in the pathway where the CoQ10 molecule moves towards COQ9 (Fig. 5f). This is realized through rotation of the Chilangle (Fig. 5g) concerted with separation of the two helices represented by the increase of the distance between W115's and A210's C $\alpha$ atoms (Fig. 5h). Once the small molecule has left the active site, W115's sidechain flips to a second stable conformation where the indole ring fills the space that the headgroup of CoQ10 was occupying (Fig. 5f,h). We thus postulated that residue W115 could act as a gate that communicates a potential pathway of substrate entry from an initial binding site around COQ9 with the active site at COQ7, and possibly also modulate separation of COQ7's helices to allow release of the product towards the membrane.

To address the relevance of this tryptophan experimentally, we mutated the corresponding W120 residue in yeast. Substitution with much smaller but also hydrophobic methionine decreased CoQ6 levels (Fig. 5i), compromised respiration (Fig. 5j) but mostly 



mutant (Fig. 51) indicates that the Trp residue is indeed important for the function of COQ7 and not its stability. Accordingly, the substitution of W120 in yeast with histidine, which also contains an aromatic ring but is more polar than the tryptophan, also distorted the production of CoQ6 (Fig. 5i-1).

Why the activity of COQ7 depends on a specific aromatic ring remains unclear.

337 role in substrate selection and loading, possibly facilitated by COQ9 and its essential quinonebinding W240 residue. At the same time, COQ7's structural similarity with alternative oxidase, a

339 protein whose function is to oxidize mature CoQ, suggests that COQ7 might require an

340 additional layer of control to promote hydroxylation of the DMQ intermediate and avoid 341 oxidation of the final CoQ product.

342 Our MD system offers novel insights and a framework for future studies of how the 343 COQ7:CO9 binds the membrane, modify its structure, and delivers a quinone intermediate to 344 COQ7.

\section{The overall COQ7:COQ9 complex forms a hetero-octameric cage.}

347 Physiologically, COQ proteins are associated with the matrix side of the inner mitochondrial

348 membrane ${ }^{53,54}$ where they can interact with each other to produce CoQ. The single-leaflet-

349 binding heterotetrameric COQ7:COQ9 fits well into this model. However, the cryoEM revealed 350 that the purified COQ7:COQ9 complex forms a soluble $\sim 240 \mathrm{kDa}$ capsid-like octamer composed 351 of two heterotetramers that associate via contacts between the helices $\alpha 7, \alpha 8$, and $\alpha 9$ of COQ9 

COQ7:COQ9 complex was higher $\left(71.6^{\circ} \mathrm{C}\right)$ possibly representing a more optimal organization of

and $\alpha 3$ and $\alpha 6$ of COQ7 (Fig. 6a). The functional significance of this assembly state remains unclear.

Globally, the octamer resembles a cube with a proteinaceous outer shell, a middle layer of protein-interacting lipids, and an inner core consisting of clearly visible densities. We identified twelve phospholipid densities occupying octamer interfaces. Our lipidomics revealed that highly abundant phospholipid species in E. coli copurified with recombinantly expressed COQ7 and COQ9 (Extended Data Fig. 10d). When considered with the structure (Fig. 3d), and the in silico simulations of membrane binding (Fig. 5a), these results suggest that lipids shield the hydrophobic surfaces of the proteins and therefore assist solubility and stability.

To test this notion, we delipidated the sample using lipid-capturing BioBeads and monitored soluble complex concentrations using SDS-PAGE gels. We observed $33 \%$ loss of the proteins upon $1 \mathrm{~h}$ incubation (Fig. 6b) without the appearance of any new oligomeric structures, as judged by Native PAGE analysis (Fig. 6c). This indicates that specific protein-lipid interactions are important for the stability of the COQ7:COQ9 complexes isolated from E. coli. In addition, we asked if protein-protein interactions also have a stabilizing effect. Therefore, we performed thermal denaturation assays to measure the stability of the lipid-rich but individually purified COQ7, COQ9, COQ7 and COQ9 combined in vitro, and the COQ7:COQ9 complex. The $T_{m}$ of the wild-type COQ9 and COQ7 were $49.1^{\circ} \mathrm{C}$ and $62.7^{\circ} \mathrm{C}$, respectively. Mixing of the proteins increased the melting temperature to $66.8^{\circ} \mathrm{C}$ which shows that COQ proteins are strongly stabilized by direct interactions in vitro. The melting temperature of the purified lipids and proteins in the complex (Fig. 6d). 
The octameric structure of the COQ7:COQ9 complex with the membrane trapped inside

375

376

377

378

379

380

381

382

sparks intriguing ideas about its potential roles, for example as a lipid transporter or an

enzymatic nano-chamber. Such functional states are rather unexpected, since the preponderance of evidence places COQ proteins only on the matrix face of the $\mathrm{IMM}^{53-56}$. However, in human and nematodes COQ7 has been reported to exist also in the nucleus ${ }^{57}$. Although these findings are debated $^{58}$, they highlight that COQ7 may have additional functions, and thus the COQ7:COQ9 octamer warrants further experimental investigation. Collectively, we conclude that our data argue in favor of the COQ7:COQ9 tetramer being a physiologically-active form of the complex that can engage lipid bilayers and whose formation depends on protein-protein and protein-lipid interactions. The significance of the octameric state will be addressed in future studies.

Quinones are highly reactive molecules ${ }^{59}$ that can be produced by cells in the form of lipids and employed as essential redox cofactors for membrane-bound enzymes ${ }^{3,60}$. However, the biosynthesis of these hydrophobic quinones presents known biochemical challenges at the aqueous/membrane interface ${ }^{61}$. Enzymes involved in this biosynthesis require simultaneous access to water-soluble cofactors and lipid-soluble substrates and need to avoid the release of immature precursors into the membrane that may lead to toxic interactions with the quinonedependent enzymes ${ }^{62}$. In the CoQ pathway, prenylation occurs at a very early step ${ }^{4}$, thereby creating a scenario whereby all subsequent intermediates are poised to partition into the membrane. How the enzymes circumvent this problem is largely unknown. 
In recent years, evidence for a complex of CoQ-related biosynthetic proteins has

that might surmount these challenges. Metabolons are dynamic protein clusters that create in the cytosol that completes a series of enzymatic reactions before returning to membrane ${ }^{70}$. CoQ metabolon whose components, the COQ proteins, are all bound to the inner mitochondrial membrane ${ }^{53,54}$. Here, by combining cryoEM, biochemical experiments, and molecular dynamics simulations, we offer the first structural insight into the actions of a complex of COQ proteins at

406 a lipid bilayer. We present structure of a substrate- and NADH cofactor-bound complex

407 comprised of the hydroxylase COQ7 and the isoprene lipid-binding protein COQ9. Our results

408 reveal how two membrane-bound proteins can cooperate to form a local membrane niche where they can retain, access, and exchange CoQ precursors.

Our data support and extend our previous model that COQ9 facilitates substrate delivery

411 to $\mathrm{COQ}^{24}$; however, the precise mechanism remains elusive. We demonstrate that the COQ7-

412 COQ9 interaction facilitates binding of the CoQ8 precursor OPP, which was enriched 50-fold

413 over background. We further reveal a novel structure ${ }^{24,71-73}$ of COQ7 with two of its helices

414 forming a membrane-interacting hydrophobic surface and a quinone-binding channel leading to

415 the protein's active site. However, in the conformation we captured by cryoEM, this channel is 416 not oriented towards COQ9 but towards the interior of the lipid bilayer. It is possible that COQ7

417 may rotate to accept the substrate from COQ9 as part of an overall cooperative mechanism;

418 however, we did not observe this in our molecular dynamics simulations. Instead, in a separate 
419 simulation, we observed that the two membrane-bound helices of COQ7 can open and permit

420 lateral quinone movement. Therefore, we speculate that COQ9 partially desorbs the quinone-

421 intermediate from the membrane and stimulates opening of COQ7's helices to allow a guided

422 lateral diffusion of the intermediate into COQ7. We further speculate that the tetrameric form of

423 the COQ7:COQ9 complex creates a proteinaceous boundary that protects the intermediates from

424 diffusing away. Interestingly, localization and dynamics of quinones in membranes are known to

425 be influenced by membrane composition and curvature ${ }^{74,75}$, and the presence of proteins ${ }^{76}$.

426 Therefore, we also hypothesize that the membrane distortion induced by the COQ7:COQ9

427 tetramer may have an additional function and help to locally retain and concentrate the quinone-

428 intermediates close to the surface of the membrane where they can be efficiently extracted and

429 modified by the enzymes.

A more complete understanding of how the COQ7:COQ9 complex functions will be

431 aided by further establishing if and how its components interact with other COQ proteins. Prior

432 work in yeast and human cells suggests a larger complex Q (or CoQ synthome) that likely

433 includes many or all proteins involved in the processing of CoQ precursors following prenylation

434 by COQ2 $2^{8,9,12,14,50,63-68}$. Gel filtration and 2D blue native-PAGE analyses of digitonin-solubilized

435 yeast mitochondria show that COQ proteins form complexes spanning a wide range of sizes with

436 increased signal detected around $66 \mathrm{kDa}, 700 \mathrm{kDa}$, and $1300 \mathrm{kDa}^{10,63},{ }^{65-68}$. It has been proposed

437 that the $700 \mathrm{kDa}$ complex lacks COQ7 and is dedicated to the production of the DMQ

438 intermediate, whereas the $1300 \mathrm{kDa}$ assembly represents the full complex that finalizes

439 production of $\mathrm{CoQ}^{77}$. Although both COQ7 and COQ9 are indeed enriched at the higher

440 molecular weight, they are also detected at the smaller one ${ }^{10,67}$. Therefore, further studies are

441 required to understand what the complexes of different sizes truly represent. It is possible that 
442 complex Q is a singular complex that directly channels quinone-intermediates between its

443 proteins, but that is not sufficiently stable to survive purification. It is also possible that COQ

444 proteins assemble into smaller complexes that co-reside at a specific region of the mitochondrial

445 membrane where they process their substrates. Intriguingly, the stability of COQ proteins and

446 their interactions seems to be positively affected by the presence of CoQ and its intermediates in

447 the membrane ${ }^{10,78}$. Moreover, fluorescent microscopy studies have shown that in vivo COQ

448 proteins assemble into CoQ-domains and colocalize with ER-mitochondria contact sites in yeast

449 (ERMES) ${ }^{8,79}$ that facilitate exchange of lipids between the organelles ${ }^{80}$. Whether complex Q and

450 CoQ-domains are one entity, and whether local membrane composition regulates CoQ

451 production remain to be established.

Although overall consistent, our results do have important limitations. Due to the use of a

453 bacterial system of protein expression, we are unable to retrieve demethoxy-coenzyme Q

454 (DMQ), the established substrate of COQ7. Instead, our structures are occupied by

455 octaprenylphenol (OPP), an early intermediate of CoQ8 production in E. coli. Moreover, COQ7

456 itself appears to be an apo-enzyme, as we do not observe any densities that could represent iron

457 atoms. Unfortunately, a parametrized model of DMQ is not available and iron has very complex

458 coordination chemistry; thus, we are not able to easily overcome these limitations using in silico

459 methods. This prohibits us from making conclusive statements about the catalytic mechanism of

460 COQ7. However, we contend that our structure presents a very plausible quinone-protein

461 arrangement that will guide more detailed studies in the future. Further work will be also

462 required to elucidate what oligomeric forms of the COQ7:COQ9 complex exist in vivo. We

463 suggest the COQ7:COQ9 heterotetramer to be the primary biological unit; however, it cannot be

464 ruled out that other assemblies of COQ7 and COQ9 have biological relevance. It is intriguing 
that the very stable COQ7:COQ9 octamer is rich in lipids but does not require detergents for

purification. How and why two membrane-binding proteins would entrap and solubilize a

467

468

469

470

471

472

473

474

475

476

477

478

479

480 portion of the plasma membrane is unknown. The membrane curving and thinning observed in the cryoEM and molecular dynamics simulations may indicate full pinching off of a part of the membrane leading to formation of an enzymatic nano-chamber in vivo. Interestingly, COQ7 has been suggested to play additional functions in the cell by participating in mtDNA homeostasis $^{81,82}$ and stress-signaling ${ }^{57,58}$. Moreover, COQ7 (called CAT5 in yeast) and YLR202C

(ORF partially overlapping with $C O Q^{9}$ ) were hits in a screen identifying loci important for sterol uptake $^{83}$. Therefore, roles for COQ7 and COQ9 beyond complex Q, and their existence in different oligomeric forms, are possibilities that will require detailed studies.

Overall, our results provide molecular insight into how peripheral membrane proteins can cooperate at a lipid membrane to selectively extract and process an extremely hydrophobic substrate. Although we focused here on CoQ biosynthesis, we anticipate that our findings will have broader utility and contribute to a better understanding of the metabolism of the rich repertoire of hydrophobic molecules residing in and creating cellular membranes.

\section{References}

1. Schoepp-Cothenet, B. et al. On the universal core of bioenergetics. Biochim Biophys Acta 1827, 79-93 (2013).

2. Nowicka, B. \& Kruk, J. Occurrence, biosynthesis and function of isoprenoid quinones. Biochimica et Biophysica Acta (BBA) - Bioenergetics 1797, 1587-1605 (2010).

3. Liu, M. \& Lu, S. Plastoquinone and Ubiquinone in Plants: Biosynthesis, Physiological Function and Metabolic Engineering. Frontiers in Plant Science 7, 1898 (2016).

4. Stefely, J.A. \& Pagliarini, D.J. Biochemistry of Mitochondrial Coenzyme Q Biosynthesis. Trends in Biochemical Sciences 42, 824-843 (2017).

5. Kemmerer, Z.A. et al. UbiB proteins regulate cellular CoQ distribution in Saccharomyces cerevisiae. Nature Communications 12, 4769 (2021).

6. Turunen, M., Olsson, J. \& Dallner, G. Metabolism and function of coenzyme Q. Biochimica et Biophysica Acta (BBA) - Biomembranes 1660, 171-199 (2004). 
7. Crane, F.L. Discovery of ubiquinone (coenzyme Q) and an overview of function. Mitochondrion 7, S2-S7 (2007).

8. Subramanian, K. et al. Coenzyme Q biosynthetic proteins assemble in a substratedependent manner into domains at ER-mitochondria contacts. Journal of Cell Biology 218, 1353-1369 (2019).

9. Floyd, Brendan J. et al. Mitochondrial Protein Interaction Mapping Identifies Regulators of Respiratory Chain Function. Molecular Cell 63, 621-632 (2016).

10. He, C.H., Xie, L.X., Allan, C.M., Tran, U.C. \& Clarke, C.F. Coenzyme Q supplementation or over-expression of the yeast Coq8 putative kinase stabilizes multisubunit Coq polypeptide complexes in yeast coq null mutants. Biochimica et Biophysica Acta (BBA) - Molecular and Cell Biology of Lipids 1841, 630-644 (2014).

11. Szklarczyk, D. et al. The STRING database in 2021: customizable protein-protein networks, and functional characterization of user-uploaded gene/measurement sets. Nucleic Acids Research 49, gkaa1074- (2020).

12. Allan, C.M. et al. Identification of Coq11, a New Coenzyme Q Biosynthetic Protein in the CoQ-Synthome in Saccharomyces cerevisiae *. Journal of Biological Chemistry 290, 7517-7534 (2015).

13. Guo, X., Niemi, N.M., Coon, J.J. \& Pagliarini, D.J. Integrative proteomics and biochemical analyses define Ptc6p as the Saccharomyces cerevisiae pyruvate dehydrogenase phosphatase. Journal of Biological Chemistry 292, 11751-11759 (2017).

14. Lohman, D.C. et al. Mitochondrial COQ9 is a lipid-binding protein that associates with COQ7 to enable coenzyme Q biosynthesis. Proceedings of the National Academy of Sciences 111, E4697-E4705 (2014).

15. García-Corzo, L. et al. Dysfunctional Coq9 protein causes predominant encephalomyopathy associated with CoQ deficiency. Human Molecular Genetics 22, 1233-1248 (2013).

16. Danhauser, K. et al. Fatal neonatal encephalopathy and lactic acidosis caused by a homozygous loss-of-function variant in COQ9. European Journal of Human Genetics 24, 450-454 (2016).

17. Smith, A.C. et al. A family segregating lethal neonatal coenzyme Q10 deficiency caused by mutations in COQ9. Journal of Inherited Metabolic Disease 41, 719-729 (2018).

18. Kwong, A.K.Y. et al. A fatal case of COQ7-associated primary coenzyme Q10 deficiency. JIMD Reports 47, 23-29 (2019).

19. Duncan, A.J. et al. A Nonsense Mutation in COQ9 Causes Autosomal-Recessive Neonatal-Onset Primary Coenzyme Q10 Deficiency: A Potentially Treatable Form of Mitochondrial Disease. The American Journal of Human Genetics 84, 558-566 (2009).

20. Olgac, A. et al. A rare case of primary coenzyme Q10 deficiency due to COQ9 mutation. Journal of Pediatric Endocrinology and Metabolism 33, 165-170 (2020).

21. Wang, Y. et al. Pathogenicity of two COQ7 mutations and responses to 2,4dihydroxybenzoate bypass treatment. Journal of Cellular and Molecular Medicine 21, 2329-2343 (2017).

22. Freyer, C. et al. Rescue of primary ubiquinone deficiency due to a novel COQ7 defect using 2,4-dihydroxybensoic acid. Journal of Medical Genetics 52, 779 (2015).

23. Wang, Y., Oxer, D. \& Hekimi, S. Mitochondrial function and lifespan of mice with controlled ubiquinone biosynthesis. Nature Communications 6, 6393 (2015). 
24. Lohman, D.C. et al. An Isoprene Lipid-Binding Protein Promotes Eukaryotic Coenzyme Q Biosynthesis. Molecular Cell 73, 763-774.e10 (2019).

25. Jonassen, T. et al. Yeast Clk-1 Homologue (Coq7/Cat5) Is a Mitochondrial Protein in Coenzyme Q Synthesis*. Journal of Biological Chemistry 273, 3351-3357 (1998).

26. Behan, R.K. \& Lippard, S.J. The Aging-Associated Enzyme CLK-1 Is a Member of the Carboxylate-Bridged Diiron Family of Proteins. Biochemistry 49, 9679-9681 (2010).

27. Andrews, S.C. The Ferritin-like superfamily: Evolution of the biological iron storeman from a rubrerythrin-like ancestor. Biochimica et Biophysica Acta (BBA) - General Subjects 1800, 691-705 (2010).

28. Huth, J.R. et al. Design of an expression system for detecting folded protein domains and mapping macromolecular interactions by NMR. Protein Science 6, 2359-2364 (1997).

29. Moore, A.L. et al. Unraveling the Heater: New Insights into the Structure of the Alternative Oxidase. Annual Review of Plant Biology 64, 637-663 (2013).

30. Aussel, L. et al. Biosynthesis and physiology of coenzyme Q in bacteria. Biochimica et Biophysica Acta (BBA) - Bioenergetics 1837, 1004-1011 (2014).

31. Jasniewski, A.J. \& Que, L. Dioxygen Activation by Nonheme Diiron Enzymes: Diverse Dioxygen Adducts, High-Valent Intermediates, and Related Model Complexes. Chemical Reviews 118, 2554-2592 (2018).

32. Lu, T.-T., Lee, S.J., Apfel, U.-P. \& Lippard, S.J. Aging-Associated Enzyme Human Clock-1: Substrate-Mediated Reduction of the Diiron Center for 5-Demethoxyubiquinone Hydroxylation. Biochemistry 52, 2236-2244 (2013).

33. Gray, H.B. \& Winkler, J.R. Electron tunneling through proteins. Quarterly Reviews of Biophysics 36, 341-372 (2003).

34. Gray, H.B. \& Winkler, J.R. Long-range electron transfer. Proc Natl Acad Sci U S A 102, 3534-9 (2005).

35. Moser, C.C., Keske, J.M., Warncke, K., Farid, R.S. \& Dutton, P.L. Nature of biological electron transfer. Nature 355, 796-802 (1992).

36. Hammes-Schiffer, S. \& Stuchebrukhov, A.A. Theory of coupled electron and proton transfer reactions. Chem Rev 110, 6939-60 (2010).

37. Sickmier, E.A. et al. X-Ray Structure of a Rex-Family Repressor/NADH Complex Insights into the Mechanism of Redox Sensing. Structure 13, 43-54 (2005).

38. Westphal, A.H. et al. Pyridine Nucleotide Coenzyme Specificity of p-Hydroxybenzoate Hydroxylase and Related Flavoprotein Monooxygenases. Frontiers in Microbiology 9 , 3050 (2018).

39. Yang, Z., Floyd, D.L., Loeber, G. \& Tong, L. Structure of a closed form of human malic enzyme and implications for catalytic mechanism. Nature Structural Biology 7, 251-257 (2000).

40. Hua, Y.H., Wu, C.Y., Sargsyan, K. \& Lim, C. Sequence-motif Detection of NAD(P)binding Proteins: Discovery of a Unique Antibacterial Drug Target. Scientific Reports 4, 6471 (2014).

41. Ansari, H.R. \& Raghava, G.P.S. Identification of NAD interacting residues in proteins. BMC Bioinformatics 11, 160 (2010).

42. Sohlenkamp, C. \& Geiger, O. Bacterial membrane lipids: diversity in structures and pathways. FEMS Microbiology Reviews 40, 133-159 (2016). 
43. Morein, S., Andersson, A.-S., Rilfors, L. \& Lindblom, G. Wild-type Escherichia coli Cells Regulate the Membrane Lipid Composition in a "Window" between Gel and Nonlamellar Structures (*). Journal of Biological Chemistry 271, 6801-6809 (1996).

44. Kaurola, P., Sharma, V., Vonk, A., Vattulainen, I. \& Róg, T. Distribution and dynamics of quinones in the lipid bilayer mimicking the inner membrane of mitochondria. Biochimica et Biophysica Acta (BBA) - Biomembranes 1858, 2116-2122 (2016).

45. Teixeira, M.H. \& Arantes, G.M. Effects of lipid composition on membrane distribution and permeability of natural quinones. RSC Advances 9, 16892-16899 (2019).

46. Katsikas, H. \& Quinn, P.J. Fluorescence Probe Studies of the Distribution of Ubiquinone Homologues in Bilayers of Dipalmitoylglycerophosphocholine. European Journal of Biochemistry 131, 607-612 (1983).

47. Hauß, T., Dante, S., Haines, T.H. \& Dencher, N.A. Localization of coenzyme Q10 in the center of a deuterated lipid membrane by neutron diffraction. Biochimica et Biophysica Acta (BBA) - Bioenergetics 1710, 57-62 (2005).

48. Ausili, A. et al. Redox State of Coenzyme Q10 Determines Its Membrane Localization. The Journal of Physical Chemistry B 112, 12696-12702 (2008).

49. Fato, R., Battino, M., Esposti, M.D., Castelli, G.P. \& Lenaz, G. Determination of partition and lateral diffusion coefficients of ubiquinones by fluorescence quenching of $n$ (9-anthroyloxy)stearic acids in phospholipid vesicles and mitochondrial membranes. Biochemistry 25, 3378-3390 (1986).

50. Yen, H.-C., Yeh, W.-Y., Lee, S.-H., Feng, Y.-H. \& Yang, S.-L. Characterization of human mitochondrial PDSS and COQ proteins and their roles in maintaining coenzyme Q10 levels and each other's stability. Biochimica et Biophysica Acta (BBA) Bioenergetics 1861, 148192 (2020).

51. Yen, H.-C. et al. Disruption of the human COQ5-containing protein complex is associated with diminished coenzyme Q10 levels under two different conditions of mitochondrial energy deficiency. Biochimica et Biophysica Acta (BBA) - General Subjects 1860, 1864-1876 (2016).

52. Galassi, V.V. \& Arantes, G.M. Partition, orientation and mobility of ubiquinones in a lipid bilayer. Biochimica et Biophysica Acta (BBA) - Bioenergetics 1847, 1560-1573 (2015).

53. Morgenstern, M. et al. Definition of a High-Confidence Mitochondrial Proteome at Quantitative Scale. Cell Reports 19, 2836-2852 (2017).

54. Vögtle, F.N. et al. Landscape of submitochondrial protein distribution. Nature Communications 8, 290 (2017).

55. Hung, V. et al. Proteomic Mapping of the Human Mitochondrial Intermembrane Space in Live Cells via Ratiometric APEX Tagging. Molecular Cell 55, 332-341 (2014).

56. Vögtle, F.N. et al. Intermembrane Space Proteome of Yeast Mitochondria*. Molecular \& Cellular Proteomics : MCP 11, 1840-1852 (2012).

57. Monaghan, R.M. et al. A nuclear role for the respiratory enzyme CLK-1 in regulating mitochondrial stress responses and longevity. Nature Cell Biology 17, 782-792 (2015).

58. Liu, J.-L., Yee, C., Wang, Y. \& Hekimi, S. A single biochemical activity underlies the pleiotropy of the aging-related protein CLK-1. Scientific Reports 7, 859 (2017).

59. Bolton, J.L., Trush, M.A., Penning, T.M., Dryhurst, G. \& Monks, T.J. Role of Quinones in Toxicology $\uparrow$. Chemical Research in Toxicology 13, 135-160 (2000). 
60. Hidalgo-Gutiérrez, A. et al. Metabolic Targets of Coenzyme Q10 in Mitochondria. Antioxidants 10, 520 (2021).

61. Forneris, F. \& Mattevi, A. Enzymes Without Borders: Mobilizing Substrates, Delivering Products. Science 321, 213-216 (2008).

62. Fernández-del-Río, L. et al. Genes and lipids that impact uptake and assimilation of exogenous coenzyme Q in Saccharomyces cerevisiae. Free Radical Biology and Medicine 154, 105-118 (2020).

63. Marbois, B., Gin, P., Gulmezian, M. \& Clarke, C.F. The yeast Coq4 polypeptide organizes a mitochondrial protein complex essential for coenzyme Q biosynthesis. Biochimica et Biophysica Acta (BBA) - Molecular and Cell Biology of Lipids 1791, 69-75 (2009).

64. Hsu, A.Y., Do, T.Q., Lee, P.T. \& Clarke, C.F. Genetic evidence for a multi-subunit complex in the O-methyltransferase steps of coenzyme Q biosynthesis. Biochimica et Biophysica Acta (BBA) - Molecular and Cell Biology of Lipids 1484, 287-297 (2000).

65. Hsieh, E.J. et al. Saccharomyces cerevisiae Coq9 polypeptide is a subunit of the mitochondrial coenzyme Q biosynthetic complex. Archives of Biochemistry and Biophysics 463, 19-26 (2007).

66. Marbois, B. et al. Coq 3 and Coq4 Define a Polypeptide Complex in Yeast Mitochondria for the Biosynthesis of Coenzyme Q*. Journal of Biological Chemistry 280, 2023120238 (2005).

67. Tran, U.C. et al. Complementation of Saccharomyces cerevisiae coq7 Mutants by Mitochondrial Targeting of the Escherichia coli UbiF Polypeptide TWO FUNCTIONS OF YEAST COQ7 POLYPEPTIDE IN COENZYME Q BIOSYNTHESIS* * This work was supported by National Institutes of Health Grant GM45952. The costs of publication of this article were defrayed in part by the payment of page charges. This article must therefore be hereby marked "advertisement" in accordance with 18 U.S.C. Section 1734 solely to indicate this fact. Journal of Biological Chemistry 281, 16401-16409 (2006).

68. Tauche, A., Krause-Buchholz, U. \& Rödel, G. Ubiquinone biosynthesis in Saccharomyces cerevisiae: the molecular organization of O-methylase Coq3p depends on Abc1p/Coq8p. FEMS Yeast Research 8, 1263-1275 (2008).

69. Pareek, V., Sha, Z., He, J., Wingreen, N.S. \& Benkovic, S.J. Metabolic channeling: predictions, deductions, and evidence. Molecular Cell 81, 3775-3785 (2021).

70. Chehade, M.H. et al. A Soluble Metabolon Synthesizes the Isoprenoid Lipid Ubiquinone. Cell Chemical Biology 26, 482-492.e7 (2019).

71. Rea, S. CLK-1/Coq7p is a DMQ mono-oxygenase and a new member of the di-iron carboxylate protein family. FEBS Letters 509, 389-394 (2001).

72. Busso, C. et al. Coq7p relevant residues for protein activity and stability. Biochimie 119, 92-102 (2015).

73. Stenmark, P. et al. A New Member of the Family of Di-iron Carboxylate Proteins. Journal of Biological Chemistry 276, 33297-33300 (2001).

74. Budin, I. et al. Viscous control of cellular respiration by membrane lipid composition. Science 362, 1186-1189 (2018).

75. Michaelis, L. \& Moore, M.J. Location of ubiquinone-10 (CoQ-10) in phospholipid vesicles. Biochimica et Biophysica Acta (BBA) - Biomembranes 821, 121-129 (1985).

76. Singharoy, A. et al. Atoms to Phenotypes: Molecular Design Principles of Cellular Energy Metabolism. Cell 179, 1098-1111.e23 (2019). 
674 77. González-Mariscal, I. et al. Regulation of coenzyme Q biosynthesis in yeast: A new

675 complex in the block. IUBMB Life 66, 63-70 (2014).

676 78. Padilla, S. et al. Hydroxylation of demethoxy-Q6 constitutes a control point in yeast

677 coenzyme Q6 biosynthesis. Cellular and Molecular Life Sciences 66, 173 (2008).

678 79. Eisenberg-Bord, M. et al. The Endoplasmic Reticulum-Mitochondria Encounter Structure

679 Complex Coordinates Coenzyme Q Biosynthesis. Contact 2, 2515256418825409 (2019).

680 80. Acoba, M.G., Senoo, N. \& Claypool, S.M. Phospholipid ebb and flow makes

681 mitochondria go. Journal of Cell Biology 219, e202003131 (2020).

682 81. Gorbunova, V. \& Seluanov, A. CLK-1 protein has DNA binding activity specific to OL

683

684 region of mitochondrial DNA. FEBS Letters 516, 279-284 (2002).

685

686

687

688

689

690

82. Kirby, C.S. \& Patel, M.R. Elevated mitochondrial DNA copy number found in ubiquinone-deficient clk-1 mutants is not rescued by ubiquinone precursor 2-4dihydroxybenzoate. Mitochondrion 58, 38-48 (2021).

83. Reiner, S., Micolod, D., Zellnig, G. \& Schneiter, R. A Genomewide Screen Reveals a Role of Mitochondria in Anaerobic Uptake of Sterols in Yeast. Molecular Biology of the Cell 17, 90-103 (2006).

\section{Methods}

\section{Site-directed mutagenesis}

693 Yeast Coq7 and Coq9 point mutants were constructed as described in the Q5® Site-Directed

694 Mutagenesis Kit (New England Biolabs) and were confirmed via Sanger sequencing. Plasmid

695 p413 harboring wild type Coq7 was used as a template. In the case of Coq9, pUC19 Coq9-FLAG

696 plasmid was used as a template and successful mutants were cloned into p416 vector using

697 EcoRI and BamHI restriction enzymes and T4 ligase (New England Biolabs). Yeast were

698 transformed as previously described ${ }^{1}$.

699

700 To generate pET24a hi6-GB1-Nd38_COQ7 the insert was amplified from pET30 GB1-

701 Nd38_COQ7 with forward primer harboring NdeI cut site and his6 tag and reverse primer

702 harboring EcoRI site. The amplicon and empty pET24a vector were cut with restriction enzymes,

703 ligated with T4 ligase, and confirmed with Sanger sequencing. Bacteria were transformed with

704 standard $30 \mathrm{sec} .42^{\circ} \mathrm{C}$ heat-shock method. 
COQ7 purification

707

708

709

710

711

712

713

714

715

716

717

718

719

720

721

722

723

724

725

726

727

E.coli Arctic Express cells harboring pET24a hi6-GB1-Nd38_COQ7 (WT or mutants) were grown $\mathrm{O} / \mathrm{N}$ at $37^{\circ} \mathrm{C}$ in $25 \mathrm{~mL} \mathrm{LB}$ media supplemented with geneticin and kanamycin. Cells were refreshed in $3 \mathrm{x} 2 \mathrm{~L} \mathrm{LB}$ to $\mathrm{OD}_{600}=0.1$ and grew with shaking at $37^{\circ} \mathrm{C}$ to $\mathrm{OD}_{600}=0.4$. Cultures were chilled to $20^{\circ} \mathrm{C}, 100 \mathrm{uM}$ ammonium iron sulfate was added, and protein expression was induced with $100 \mu \mathrm{M}$ Isopropyl $\beta$ - d-1-thiogalactopyranoside (IPTG). After the first and the second hour $100 \mu \mathrm{M}$ ammonium iron sulfate was added again. Protein production was continued for $24 \mathrm{~h}$ total and then cells were spun $10 \mathrm{~min} . \mathrm{x} 5000 \mathrm{~g}$ at $4^{\circ} \mathrm{C}$. Each $15 \mathrm{~g}$ of cell paste were resuspended in 35 $\mathrm{mL}$ total Lysis Buffer (20 mM HEPES pH 7.5, $200 \mathrm{mM} \mathrm{NaCl}, 10 \%$ glycerol, $5 \mathrm{mM}$ sodium thioglycolate, $1 \mathrm{mM}$ cysteine, $1 \mu \mathrm{L}$ Benzonase, $5 \mathrm{mM} \mathrm{MgCl} 2,500 \mu \mathrm{M}$ phenylmethylsulfonyl fluoride (PMSF), $0.1 \mathrm{mg} / \mathrm{mL}$ lysozyme) and incubated at $4{ }^{\circ} \mathrm{C}$ with slow rotation. Next, the conical tubes were put on ice and the cells were lysed using Bronson sonifier (50\% amplitude, 10 sec. ON/60 sec. OFF, 3 cycles). The lysate was spun (10 min. x 50 000g), supernatant was collected and the soluble fraction of COQ7 was subjected to IMAC purification on TALON resin as described later. The pelleted fraction containing membrane-bound COQ7 was resuspended in 10 $\mathrm{mL}$ of Lysis Buffer supplemented with 3\% digitonin. Sample was loaded into syringe and further homogenized by passing it several times through a needle. The homogenate was incubated at $4^{\circ} \mathrm{C}$ with rotation for next $2 \mathrm{~h}$. Then, it was transferred to oakridge tube and Lysis Buffer was added to $30 \mathrm{~mL}$ total. Sample was spun $1 \mathrm{~h}$ x $50000 \mathrm{~g}$ and supernatant containing solubilized proteins was loaded onto $5 \mathrm{~mL}$ of TALON resin equilibrated with Wash Buffer (20 mM HEPES 7.5, $200 \mathrm{mM}$ $\mathrm{NaCl}, 10 \%$ glycerol, $1 \mathrm{mM}$ imidazole) in $50 \mathrm{~mL}$ conical tube. After $2 \mathrm{~h}$ incubation at $4^{\circ} \mathrm{C}$ with gentle rotation, the resin was washed 3 times with $50 \mathrm{~mL}$ of Wash Buffer $+10 \mathrm{mM}$ imidazole. 
728 Next, the resin was transferred to $15 \mathrm{~mL}$ conical tube and the COQ7 was eluted with $5 \mathrm{~mL}$ of

729 Elution Buffer (20 mM HEPES 7.5, $200 \mathrm{mM} \mathrm{NaCl}, 10 \%$ glycerol, $150 \mathrm{mM}$ imidazole). Sample

730 was incubated in ice for $10 \mathrm{~min}$, , spun $5 \mathrm{~min} . \mathrm{x} 700 \mathrm{~g}$, supernatant was collected, and the resin was

731 subjected to another round of elution. Supernatants were combined, concentrated to $1 \mathrm{~mL}$ using

732 Amicon Centrifugal Filter (15k Da cut-off) and subjected to Size Exclusion Chromatography. Best

733 fractions were pooled together, frozen with liquid nitrogen and stored at $-80 \mathrm{C}$.

734

735

COQ9 and COQ7:COQ9 complex purification

736 E.coli Arctic Express cells harboring pETduet his6-Nd79_COQ9 alone or co-transformed with pET30 GB1-Nd38_COQ7 were grown $\mathrm{O} / \mathrm{N}$ at $37^{\circ} \mathrm{C}$ in $25 \mathrm{~mL} \mathrm{LB}$ media supplemented with geneticin, ampicillin and kanamycin as needed. Cells were refreshed in $3 \mathrm{x} 2 \mathrm{~L}$ LB to $\mathrm{OD}_{600}=0.1$ and grew with shaking at $37^{\circ} \mathrm{C}$ to $\mathrm{OD}_{600}=0.4$. Cultures were chilled to $20^{\circ} \mathrm{C}, 100 \mu \mathrm{M}$ ammonium

740 iron sulfate was added, and protein expression was induced with $100 \mu \mathrm{M}$ Isopropyl $\beta$ - d-1thiogalactopyranoside (IPTG). After the first and the second hour $100 \mu \mathrm{M}$ ammonium iron sulfate was added again. Protein production was continued for $24 \mathrm{~h}$ total and then cells were spun $10 \mathrm{~min}$.

$743 \times 5000 \mathrm{~g}$ at $4^{\circ} \mathrm{C}$. Each $15 \mathrm{~g}$ of cell paste was resuspended in $35 \mathrm{~mL}$ total Lysis Buffer $(20 \mathrm{mM}$ 744 HEPES pH 7.5, $200 \mathrm{mM} \mathrm{NaCl}, 10 \%$ glycerol, $5 \mathrm{mM}$ sodium thioglycolate, $1 \mathrm{mM}$ cysteine, $1 \mu \mathrm{L}$ 745 Benzonase, $5 \mathrm{mM} \mathrm{MgCl}_{2}, 500 \mu \mathrm{M}$ phenylmethylsulfonyl fluoride (PMSF), $0.1 \mathrm{mg} / \mathrm{mL}$ lysozyme) and incubated at $4{ }^{\circ} \mathrm{C}$ with slow rotation. Next, the conical tubes were put on ice and the cells were

747 lysed using Bronson sonifier (50\% amplitude, $10 \mathrm{sec}$. ON/60 sec. OFF, 3 cycles). The lysate was spun $(30 \mathrm{~min} . \mathrm{x} 50000 \mathrm{~g})$ and the supernatant was loaded onto $5 \mathrm{~mL}$ of TALON resin equilibrated with Wash Buffer (20 mM HEPES 7.5, $200 \mathrm{mM} \mathrm{NaCl,} \mathrm{10 \%} \mathrm{glycerol,} 1 \mathrm{mM}$ imidazole) in $50 \mathrm{~mL}$ conical tube. After $2 \mathrm{~h}$ incubation at $4^{\circ} \mathrm{C}$ with gentle rotation, the resin was washed 3 times with 
$50 \mathrm{~mL}$ of Wash Buffer $+10 \mathrm{mM}$ imidazole. Next, the resin was transferred to $15 \mathrm{~mL}$ conical tube and proteins were eluted with $5 \mathrm{~mL}$ of Elution Buffer $(20 \mathrm{mM}$ HEPES 7.5, 200mM NaCl, 10\% glycerol, $150 \mathrm{mM}$ imidazole). Sample was incubated in ice for $10 \mathrm{~min}$., spun $5 \mathrm{~min} . \mathrm{x} 700 \mathrm{~g}$,

754 supernatant was collected, and the resin was subjected to another round of elution. Supernatants 755 were combined, concentrated to $1 \mathrm{~mL}$ using Amicon Centrifugal Filter (15k Da cut-off) and 756 subjected to Size Exclusion Chromatography and subsequent blue native PAGE analysis 757 (NativePAGE, 4-16\%, Bis-Tris, Invitrogen).

759 Size Exclusion Chromatography

760 Purified proteins concentrated to $1 \mathrm{~mL}$ or gel filtration standards (BioRad, 1511901) were injected 761 on to Hi-Load pg200 column and $150 \mathrm{~mL}$ eluate was collected as $1.0 \mathrm{~mL}$ fractions at $1.0 \mathrm{~mL} / \mathrm{min}$., 762 in $20 \mathrm{mM}$ HEPES pH 7.5, $200 \mathrm{mM} \mathrm{NaCl}$ buffer or in $20 \mathrm{mM}$ ammonium acetate $\mathrm{pH} 7.0$ in case of 763 the COQ7:COQ9 complex subjected later to the cryoEM. UV absorbance signal was used to detect 764 protein-containing fractions which were then analyzed by SDS-PAGE (NuPAGETM 4 to $12 \%$, Bis765 Tris, Invitrogen) to assess their purity. Best fractions were pooled together, frozen with liquid 766 nitrogen and stored at $-80^{\circ} \mathrm{C}$.

\section{Lipids extraction from yeast}

769 Yeast were transformed with p413 or p416 plasmids carrying wild type or mutated version of yeast 770 COQ7 and COQ9 genes, respectively. Next, individual colony was picked from the S.C. 2\% 771 glucose pABA- plate and used to start $3 \mathrm{~mL}$ liquid culture in the same media. After $12 \mathrm{~h}$ growth at $77230^{\circ} \mathrm{C}$ with shaking, the cultures were used to prepare new $3 \mathrm{~mL}$ cultures in S.C. $0.1 \%$ glucose, $773 \quad 3.0 \%$ glycerol, $50 \mathrm{nM}$ pABA at $\mathrm{OD}_{600}=0.1$ and incubated at $30 \mathrm{C}$ with shaking for $24 \mathrm{~h}$ to pass the 
774 diauxic shift. Next, equivalent of $5 \mathrm{~mL} \mathrm{OD}_{600}=1.0(\sim 5 \mathrm{e} 7$ cells $)$ was pelleted $(2 \mathrm{~min} . \mathrm{x} 3000 \mathrm{~g})$ in

$7751.5 \mathrm{~mL}$ screw-cap tube. The supernatant was discarded, $100 \mu \mathrm{L}$ of glass beads, and $50 \mu \mathrm{L}$ of 150

$776 \mathrm{mM} \mathrm{KCl}$, and $600 \mu \mathrm{L}$ of methanol with $0.1 \mu \mathrm{M} \mathrm{CoQ}_{8}$ internal standard (Avanti Polar Lipids) were

777 added, and the cells were lysed by 2 rounds of $5 \mathrm{~min}$. bead-beating in disruptor genie set to $\max$

778 (3000 rpm) speed. To extract lipids, $400 \mu \mathrm{L}$ of petroleum ether was added to sample and subjected

779 to bead-beating for $3 \mathrm{~min}$. Sample was then spun $2 \mathrm{~min} . \mathrm{x} 1000 \mathrm{~g}$ at $4^{\circ} \mathrm{C}$ and the ether layer (top)

780 was transferred to a new tube. Extraction was repeated, the ether layers were combined and dried

781 ( 30 min.) under argon gas at room temperature.

782

\section{CoQ6 measurement by HPLC-ECD}

784 Extracted dried lipids were resuspended in $50 \mu \mathrm{L}$ of mobile phase (78\% methanol. 20\% isopropanol, 2\% $1 \mathrm{M}$ ammonium acetate $\mathrm{pH} 4.4$ in water and transferred to amber glass vials with inserts. Vials were inserted into HPLC (Ultimate 3000, Thermo Scientific) with electrochemical detector (ECD-3000RS) and $10 \mu \mathrm{L}$ of each sample was injected. The flow rate was set to 0.3 $\mathrm{mL} / \mathrm{min}$ (LPG-3400RS pump). The first electrode (6020RS) was set to $+600 \mathrm{mV}$ and placed before the column (Thermo Scientific, Betasil C18, $100 \times 2.1 \mathrm{~mm}$, 3um particle) to oxidize all the

791 reduce the quinones exiting the column, and then the third electrode was set to $+600 \mathrm{mV}$ to make

792 final recordings. A CoQ 6 standard (Avanti Polar Lipids) was used to identify corresponding peak 793 in the obtained data. Peaks were then quantified with Chromeleon 7.2.10 software using cobra 794 wizard option. 
797 Samples were prepared according to "NADH fluorescence method". After 30 min. incubation 50

$798 \mu \mathrm{L}$ of each sample was quenched with $50 \mu \mathrm{L}$ of $-20^{\circ} \mathrm{C}$ cold methanol, incubated at $-20^{\circ} \mathrm{C}$ for 5

$799 \mathrm{~min}$. and spun at $2^{\circ} \mathrm{C}$ for $5 \mathrm{~min}$. x $20000 \mathrm{~g}$. Supernatants from triplicates were pooled together and

800 transferred to amber glass vials. Vials were inserted into HPLC (Ultimate 3000, Thermo Scientific)

801 with electrochemical detector (ECD-3000RS) and cooled to $4^{\circ} \mathrm{C}$. Then, $2.5 \mu \mathrm{L}$ of each sample was

802 injected and resolved at $0.25 \mathrm{~mL} / \mathrm{min}$. (LPG-3400RS pump) isocratic flow of $90 \% \mathrm{~A}(0.1 \%$ formic

803 acid in $\mathrm{H}_{2} \mathrm{O}$ ) and 10\% B (isopropanol) mobile phase. The first electrode (6020RS) placed before

804 the column (BetaBasic C18, $100 \times 4.5 \mathrm{~mm}$, 5um particle) was turned off $(0 \mathrm{mV})$. Second electrode

805 (6011RS) was set to $-600 \mathrm{mV}$ to reduce the quinones exiting the column, and then the third

806 electrode was set to $+600 \mathrm{mV}$ to make final recordings. Data were compared to $250 \mu \mathrm{M} \mathrm{DMQ}_{0}$

807 standard (WuXi AppTec) pre-oxidized with first electrode set to $+600 \mathrm{mV}$ or pre-reduced with the

808 electrode set to $-600 \mathrm{mV}$.

809

810 Wester Blotting

811 Methanol-glass beads mix from the lipid extraction protocol was dried under fume hood at $60^{\circ} \mathrm{C}$

812 for several hours until complete dryness. Next, $200 \mu \mathrm{L}$ of 1x LDS Buffer with 10 mM DTT was

813 added and the sample was boiled for $20 \mathrm{~min}$. with often intense vortexing. Beads were spun 5

$814 \min . \times 20000 \mathrm{~g}$ and $15 \mu \mathrm{L}$ of supernatant was loaded on SDS-PAGE gel (NuPAGETM 4 to $12 \%$,

815 Bis-Tris, Invitrogen). Proteins were electrophoretically separated for $35 \mathrm{~min}$. at 150V, and then

816 transferred (192 mM glycine, $25 \mathrm{mM}$ Tris, 20\% methanol [v/v]) to methanol-activated PVDF

817 membrane (Immobilon-FL). The membrane was washed with TBS-T (20 mM Tris pH 7.4,

$818150 \mathrm{mM} \mathrm{NaCl}, 0.05 \%$ Tween $20[\mathrm{v} / \mathrm{v}])$, blocked with 5\% powdered milk in TBS-T, washed 3

819 times with TBS-T, incubated at $4^{\circ} \mathrm{C} \mathrm{O} / \mathrm{N}$ with primary $\alpha$-FLAG (Millipore F1804-5MG) or $\alpha$ - 
820 beta-Actin (Abcam ab8224) or $\alpha$-Coq7 (raised by GenScript in rabbits against

821 NLERTDGTKGPSEE peptide) antibodies (1:5000 in 0.5\% milk), washed 3 times with TBS-T,

822 incubated with secondary antibodies (1:20 000 in $0.1 \%$ milk, goat anti-mouse (LI-COR 926-

823 32210, 1:15000; RRID: AB_621842) or goat anti-rabbit (LI-COR 926-32211, 1:15000; RRID:

824 AB_621843)), washed 3 times with TBS-T, and finally visualized with LI-COR Odyessey CLx 825 scanner using Image Studio v5.2 software.

\section{$827 \quad$ Yeast respiratory growth}

828 Individual colony was picked from the S.C. -URA or -HIS, 2\% glucose, pABA-, plate and used to 829 start $3 \mathrm{~mL}$ liquid culture in the same media. After $12 \mathrm{~h}$ growth at $30^{\circ} \mathrm{C}$ with shaking, the culture 830 was used to prepare new $1.0 \mathrm{~mL}$ culture in S.C. $0.1 \%$ glucose, $3.0 \%$ glycerol, $50 \mathrm{nM}$ pABA at $831 \mathrm{OD}_{600}=0.05$. Next, $100 \mu \mathrm{L}$ of it was transferred to 96-well round-bottom clear plate (Thermo) and 832 sealed with BreatheEasy film (USA Scientific). The plate was loaded into EPOCH plate reader operated with Gen5 3.1 software and yeast were grown at $30^{\circ} \mathrm{C}$ with max linear shaking (1096

$834 \mathrm{cpm}$ ) for 48-72h with optical density monitored every $20 \mathrm{~min}$. at $600 \mathrm{~nm}$.

COQ7:COQ9 complex delipidation

$837100 \mu \mathrm{L}$ of COQ9:COQ7 complex was thawed (20mM HEPES pH $7.5200 \mathrm{mM} \mathrm{NaCl}, 10 \%$

838 glycerol at $1.5 \mu \mathrm{g} / \mu \mathrm{L}$ ) and incubated $1 \mathrm{~h}$ with $100 \mu \mathrm{L}$ BioBeads (washed with $1 \mathrm{~mL}$ of methanol 839 and then $3 \times 1 \mathrm{~mL}$ buffer) at room temp with orbital shaking. In parallel, $100 \mu \mathrm{L}$ of COQ9:COQ7

840 was incubated without biobeads as a control of protein stability at the room temp. $5 \mu \mathrm{L}$ was

841 collected after $1 \mathrm{~h}$. The beads were spun $15000 \mathrm{~g}$ x $1 \mathrm{~min}$. and the supernatant containing

842 delipidated complex was transferred to a new tube. The beads were washed 3 times with $1 \mathrm{~mL}$ of 
843 the buffer and resuspended in $100 \mu \mathrm{L}$ of $1 \mathrm{x}$ LDS buffer with $5 \mathrm{mM}$ DTT. Finally, blue native

844 PAGE (NativePAGE, 4-16\%, Bis-Tris, Invitrogen) and SDS-PAGE (NuPAGETM 4 to 12\%, Bis-

845 Tris, Invitrogen) were run to analyze results.

846

847 NADH fluorescence

848 Buffer (20 mM HEPES pH 7.6, $200 \mathrm{mM} \mathrm{NaCl})$ was mixed with $5 \mu \mathrm{M}$ protein, $250 \mu \mathrm{M}$ NADH, 849 and $250 \mu \mathrm{M} \mathrm{DMQ}_{0}$ as indicated in the figure to total reaction volume of $100 \mu \mathrm{L}$. Samples were

850 loaded into black flat clear bottom 96-well plate (BRANDplates, pure-grade) and incubated at

$85130^{\circ} \mathrm{C}$ with constant slow orbital shaking. Optics were set to TOP position with probe's height at

$8525 \mathrm{~mm}$ and gain value 100. Then, NADH's fluorescence was recorded every $5 \mathrm{~min}$. (Excitation= $853340 \mathrm{~nm}$, Emission=445 nm) for $4 \mathrm{~h}$.

854

855 Differential Fluorimetry Scanning (DSF)

$85624.5 \mu \mathrm{L}$ of $5 \mu \mathrm{M}$ solution of every protein was prepared in 96-well plate MicroAmp Optical plate

857 (Applied biosystems), and incubated at room temp. for $15 \mathrm{~min}$. Then, $0.5 \mu \mathrm{L} 50 \mathrm{x}$ SYPRO

858 Orange (Invitrogen) dye in 10\% DMSO was added to each well and mixed by pipetting. The

859 plate was sealed with MicroAmp Optical film (Applied biosystems), spun 1 min. x $1000 \mathrm{~g}$,

860 loaded into QuantStudio 6 Flex (Applied biosystems) and subjected to $20-99^{\circ} \mathrm{C}$ heat gradient

861 changing at $0.025^{\circ} \mathrm{C} / \mathrm{sec}$. Signal for ROX dye (overlapping with SYPRO orange) was recorded.

862 The data were analyzed using DSFworld server (https://gestwickilab.shinyapps.io/dsfworld). Fits

8632 and 4 were selected as the best representing the shape of melting curves and used to calculate

864 melting temperatures. 


\section{In silico analyses}

867 Tunnels in COQ7 were detected with MOLEonline ${ }^{2}$ server (https://mole.upol.cz/) using default

868 settings. All graphs were prepared using GraphPad Prism 9.1.0 software. All structures were

869 analyzed (inter-residue distances, interactions etc.) and visualized using CHIMERA 1.11.2

870 software $^{3}$. Electrostatic surface potentials of proteins were calculated using APBS server ${ }^{4}$

871 (poissonboltzmann.org) on default settings. Densitometry was performed with ImageJ $1.50 \mathrm{i}^{5}$.

872 Student t-tests were calculated in Excel.

873

Mass-spec lipidomics analysis of DMQ6 content in yeast.

875 Lipid extracts from equivalents of $5 \mathrm{~mL} \mathrm{OD}_{600}=1.0(\sim 5 \mathrm{e} 7$ cells $)$ yeast cultures were prepared as

876 described in "HPLC-ECD” section. LC-MS analysis was performed using Thermo Vanquish

877 Horizon UHPLC system coupled to a Thermo Exploris 240 Orbitrap mass spectrometer. For LC

878 separation, A Vanquish binary pump system (Thermo Scientific) was used with a Waters

879 Acquity CSH C18 column $(100 \mathrm{~mm} \times 2.1 \mathrm{~mm}, 1.7 \mathrm{~mm}$ particle size $)$ held at $35^{\circ} \mathrm{C}$ under 300

$880 \mu \mathrm{L} / \mathrm{min}$ flow rate. Mobile phase A consisted of $5 \mathrm{mM}$ ammonium acetate in $\mathrm{ACN} / \mathrm{H}_{2} \mathrm{O}(70: 30$,

$881 \mathrm{v} / \mathrm{v}$ ) containing $125 \mu \mathrm{L} / \mathrm{L}$ acetic acid. Mobile phase B consisted of $5 \mathrm{mM}$ ammonium acetate in

882 IPA/ACN $(90: 10, v / v)$ with the same additive. For each sample run, mobile phase B was initially

883 held at $2 \%$ for $2 \mathrm{~min}$ and then increased to $30 \%$ over $3 \mathrm{~min}$. Mobile phase B was further

884 increased to $50 \%$ over $1 \mathrm{~min}$ and $85 \%$ over $14 \mathrm{~min}$ and then raised to $99 \%$ over 1 min and held

885 for $4 \mathrm{~min}$. The column was re-equilibrated for $5 \mathrm{~min}$ at $2 \% \mathrm{~B}$ before the next injection. Five

886 microliters of sample were injected by a Vanquish Split Sampler HT autosampler (Thermo

887 Scientific) while the autosampler temperature was kept at $4{ }^{\circ} \mathrm{C}$. The samples were ionized by a

888 heated ESI source kept at a vaporizer temperature of $350^{\circ} \mathrm{C}$. Sheath gas was set to 50 units, 
889

890

891

892

893 $\mathrm{MS}^{2}$ acquisitions.

900 Scientific).

904

905

906

907

908

909

910

911

\section{Data Analysis}

auxiliary gas to 8 units, sweep gas to 1 unit, and the spray voltage was set to $3,500 \mathrm{~V}$ for positive mode and 2,500 V for negative mode (two separate injections per replicate). The inlet ion transfer tube temperature was kept at $325^{\circ} \mathrm{C}$ with $70 \%$ RF lens. For discovery analysis, $\mathrm{MS}^{1}$ scans were acquired at 120,000 resolution from m/z 200 to 1,700 with EasyIC enabled to improve mass accuracy. $\mathrm{MS}^{2}$ scans were acquired in AcquireX Background Exclusion mode to automatically exclude background ions found in the extraction blank from MS/MS fragmentation during sample acquisitions with the cycle time of $1.5 \mathrm{~s}$. Other $\mathrm{MS}^{2}$ parameters include resolution of 30,000, $54 \mathrm{~ms} \mathrm{MS} \mathrm{MS}^{2}$ ion injection time, $1.5 \mathrm{~m} / \mathrm{z}$ isolation width, stepped HCD collision energy (25\%, 30\% for positive mode and $20 \%, 40 \%, 60 \%$ for negative mode), and $3 \mathrm{~s}$ dynamic exclusion. Automatic gain control (AGC) targets were set to standard mode for both $\mathrm{MS}^{1}$ and

The resulting CoQ intermediate data were processed using TraceFinder 5.1 (Thermo Fisher

\section{LC-MS lipidomics of purified proteins and $E$. coli cells.}

For lipidomics analysis of COQ7:COQ9 complex, two $20 \mathrm{uL}$ aliquots of $5 \mathrm{mg} / \mathrm{mL}$ protein COQ9:COQ7 complex, in $200 \mathrm{mM}$ ammonium Acetate were extracted using Matyash method ${ }^{6}$. Briefly, once samples were thawed on ice, $200 \mu \mathrm{L}$ methyl tert-butyl ether (MTBE), $60 \mathrm{uL}$ of methanol, and $50 \mathrm{uL}$ of water was added to each tube and the mixture was vortexed for $10 \mathrm{~s}$. The sample was then centrifuged for $10 \mathrm{~min}$ at $10,000 \mathrm{~g}$ at $4^{\circ} \mathrm{C} .150 \mu \mathrm{L}$ of the lipophilic (upper) layer 911 from the biphasic extraction was aliquoted into a separate glass vial and dried down by vacuum 
912 centrifugation, and was then resuspended in $50 \mu \mathrm{L}$ 9:1 $\mathrm{MeOH} /$ Toluene prior to LC-MS/MS

913 analysis.

914 For lipidomics of the separately purified proteins, two aliquots of each purified protein $(100 \mu \mathrm{g}$

915 COQ7, $100 \mu \mathrm{g}$ COQ9, $200 \mu \mathrm{g}$ COQ7:COQ9) or pelleted E.coli cells (10e10, $20 \times$ OD $\left._{600}=1.0\right)$

916 were thawed on ice. Once thawed, $87 \mu \mathrm{L}$ of methanol, and $290 \mu \mathrm{L}$ methyl tert-butyl ether (MTBE)

917 were added to each non-pellet tube and the mixture was vortexed for $10 \mathrm{~s}$ (sample contained 100

$918 \mu \mathrm{L}$ of aqueous buffer). For each of the $E$. coli cell pellet tubes, $205 \mu \mathrm{L}$ methanol, $750 \mu \mathrm{L}$ MTBE,

919 and $187.5 \mu \mathrm{L}$ water were added, and the mixture was sonicated for $10 \mathrm{~s}$. After centrifugation for 2

$920 \mathrm{~min}$ at $14,000 \mathrm{~g}$ at $4^{\circ} \mathrm{C}, 200 \mu \mathrm{L}$ of the lipophilic (upper) layer from the biphasic extraction was

921 aliquoted into an amber glass autosampler vial with glass insert and dried down by vacuum

922 centrifugation. The samples were then resuspended in $50 \mu \mathrm{L}$ 9:1 MeOH/toluene prior to LC-

$923 \mathrm{MS} / \mathrm{MS}$ analysis.

924

LC-MS/MS analysis for lipidomics.

926 Sample analysis was performed using an Acquity CSH C18 column held at $50{ }^{\circ} \mathrm{C}(100 \mathrm{~mm} \times 2.1$

$927 \mathrm{~mm} \times 1.7 \mu \mathrm{m}$ particle size; Waters), a $400 \mu \mathrm{L} / \mathrm{min}$ flow rate was maintained using a Vanquish

928 Binary Pump (Thermo Scientific). The mobile phases consisted of $10 \mathrm{mM}$ ammonium acetate in

929 ACN:H2O (70:30, v/v) with $250 \mu \mathrm{L} / \mathrm{L}$ acetic acid (Mobile Phase A), and $10 \mathrm{mM}$ ammonium

930 acetate in IPA:ACN (90:10, v/v) with $250 \mu \mathrm{L} / \mathrm{L}$ acetic acid (Mobile Phase B). For each sample 10

$931 \mu \mathrm{L}$, was injected onto column using Vanquish autosampler (Thermo Scientific). The gradient for

932 lipid separations was as follows, mobile phase B was initially held at $2 \%$ for 2 min and then

933 increased to $30 \%$ over $3 \mathrm{~min}$. Mobile phase B was further increased to $50 \%$ over 1 min, then raised 
to $85 \%$ over $14 \mathrm{~min}$, and finally raised to $99 \%$ over $1 \mathrm{~min}$ and held at $99 \%$ for $7 \mathrm{~min}$. The column was re-equilibrated with mobile phase $\mathrm{B}$ at $2 \%$ for $1.75 \mathrm{~min}$ before the next injection.

The LC system was coupled to a Q Exactive Orbitrap mass spectrometer through a heated electrospray ionization (HESI II) source (Thermo Scientific). Source conditions were as follow: HESI II and capillary temperature at $300{ }^{\circ} \mathrm{C}$, sheath gas flow rate at 25 units, aux gas flow rate at 15 units, sweep gas flow rate at 5 units, spray voltage at $|3.5 \mathrm{kV}|$ for both positive and negative modes, and S-lens RF at 90.0 units. Data were acquired using polarity switching with positive and negative full MS and MS2 spectra (Top2) within the same injection. Acquisition parameters for full MS scans in both modes were 17,500 resolution, $1 \times 10^{6}$ automatic gain control (AGC) target, $100 \mathrm{~ms}$ max inject time, and 200 to $1600 \mathrm{~m} / \mathrm{z}$ scan range. MS2 scans in both modes were then performed at 17,500 resolution, $1 \times 10^{5}$ AGC target, $50 \mathrm{~ms} \max$ IT, $1.0 \mathrm{~m} / \mathrm{z}$ isolation window, stepped normalized collision energy (NCE) at 20, 30, 40, and a 10.0 s dynamic exclusion

Data Analysis for lipidomics by mass spectrometry

LC-MS data were processed using Compound Discoverer 2.1 and 3.1 (Thermo Scientific) and LipiDex, an in-house-developed software suite ${ }^{7}$. Features detection was performed within a 1.4 min to 21 min retention time window, and features were aggregated into compound groups using

951 a 10-ppm mass and 0.75 min retention time tolerance. Detect compounds node settings of 952 minimum peak intensity of $5 \times 10^{5}$, maximum peak-width of 0.75 , and signal-to-noise $(\mathrm{S} / \mathrm{N})$ ratio 953 of 3 were used. Background features were designated as features less than 3-fold intensity over 954 blanks and were excluded from further processing. MS/MS spectra were searched against an in955 silico generated lipid spectral library ${ }^{7}$. Spectral matches with a dot product score greater than 500 956 and a reverse dot product score greater than 700, eluting within a 3.5 median absolute retention 
957 time deviation (M.A.D. RT) of each other, and found within at least 2 processed files were retained.

958 Lipids with no significant interference $(<75 \%)$ from co-eluting isobaric lipids were identified at

959 the individual fatty acid substituent level, otherwise lipids were annotated with the sum of the fatty

960 acid substituents.

961

962 In-gel sample digestion for proteomics

963 All the steps were performed at room temperature with use of LC-MS grade reagents. Samples

964 were loaded onto reducing SDS-PAGE gel (NuPAGETM 4 to 12\%, Bis-Tris, Invitrogen) and

965 resolved $45 \mathrm{~min} . \mathrm{x} 150 \mathrm{~V}$. Individual bands were excised from the gel, placed in separate tubes

966 and cut into smaller pieces. Samples were destined for $15 \mathrm{~min}$. in $100 \mu \mathrm{L}$ of $25 \mathrm{mM}$ ammonium

967 bicarbonate (AMBIC) in acetonitrile/water $(50 / 50, \mathrm{v} / \mathrm{v})$ buffer. Next, samples were washed for

$96810 \mathrm{~min}$. with acetonitrile and air dried for $10 \mathrm{~min}$. Then, samples were reduced $(40 \mu \mathrm{L}$ of $10 \mathrm{mM}$

969 TCEP and $40 \mathrm{mM}$ 2-chloroacetemide in $25 \mathrm{mM}$ AMBIC) for $30 \mathrm{~min}$. and dehydrated in $200 \mu \mathrm{L}$

970 acetonitrile for $10 \mathrm{~min}$. The gel pieces were air dried for $10 \mathrm{~min}$., incubated with $40 \mu \mathrm{L}$ of trypsin

971 (Promega, Sequencing Grade, $16 \mathrm{ng} / \mu \mathrm{L}$ in $25 \mathrm{mM}$ AMBIC) for $15 \mathrm{~min}$, , supplemented with

972 additional $30 \mu \mathrm{L}$ of $25 \mathrm{mM}$ AMBIC and the proteins were digested overnight. Supernatants

973 containing digested peptides were transferred to new tubes and gel pieces were covered with 100

$974 \mu \mathrm{L}$ extraction buffer $(5 \%$ formic acid in water/acetonitrile $(1 / 2, \mathrm{v} / \mathrm{v})$, incubated for $10 \mathrm{~min}$. and

975 the supernatants were collected and combined with the previous ones. Samples were dried down

976 to several $\mu \mathrm{L}$ in Speedvac (Thermo Scientific), resuspended in $100 \mathrm{uL} 0.1 \%$ TFA and desalted

977 using C18 Omix tips (Agilent, \#A57003100K). Each time a tip was conditioned with 2x $100 \mu \mathrm{L}$

978 of $0.1 \%$ TFA in 50:50 (v/v) $\mathrm{ACN} / \mathrm{H}_{2} \mathrm{O}$, washed with $2 \mathrm{x} 100 \mu \mathrm{L}$ of $0.1 \%$ TFA in $\mathrm{H}_{2} \mathrm{O}$, sample

979 was bound by passing 10 times thru tip resin, and then washed $3 \times 100 \mu \mathrm{L}$ of $0.1 \%$ TFA in $\mathrm{H}_{2} \mathrm{O}$. 
980

981

982

983

984

985

986

987

988

989

990

991

992

993

994

995

996

997

998

999

1000

1001

1002

The peptides were eluted with $100 \mu \mathrm{L}$ of 0.1\% TFA in 80:20 (v/v) ACN/ $\mathrm{H}_{2} \mathrm{O}$, dried in Speedvac, reconstituted in $20 \mu \mathrm{L}$ of $0.2 \%$ formic acid in $\mathrm{H}_{2} \mathrm{O}$ and subjected to $\mathrm{LC}-\mathrm{MS}$ analysis.

\section{Liquid Chromatography Mass Spectrometry Proteomics}

LC separation was performed using Thermo Ultimate 3000 RSLCnano system. A $15 \mathrm{~cm}$ EASYSpray $^{\text {TM }}$ PepMap ${ }^{\text {TM }}$ RSLC C18 column $(150 \mathrm{~mm} \times 75 \mu \mathrm{m}, 3 \mu \mathrm{m})$ was used at $300 \mathrm{~nL} / \mathrm{min}$ flow rate with a 60 min. gradient using mobile phase A consisting of $0.1 \%$ formic acid in $\mathrm{H}_{2} \mathrm{O}$, and mobile phase B consisting of $0.1 \%$ formic acid in $\mathrm{ACN} / \mathrm{H}_{2} \mathrm{O}(80 / 20, \mathrm{v} / \mathrm{v})$. EASY-Spray source was used and temperature was at $35^{\circ} \mathrm{C}$. Each sample run was held at $4.0 \% \mathrm{~B}$ for 3 min and increased to $45 \% \mathrm{~B}$ over $42 \mathrm{~min}$, followed by $5 \mathrm{~min}$ at $95 \% \mathrm{~B}$ and back to $4 \% \mathrm{~B}$ for equilibration for 10 min. An Acclaim PepMap C18 HPLC trap column $(20 \mathrm{~mm} \times 75 \mu \mathrm{m}, 3 \mu \mathrm{m})$ was used for sample loading. MS detection was performed with Thermo Exploris 240 Orbitrap mass spectrometer in positive mode. The source voltage was set to $1.5 \mathrm{kV}$, ion transfer tube temperature was set to $275^{\circ} \mathrm{C}$, RF lens was at $40 \%$. Full MS spectra were acquired from m/z 350

to 1400 at the Orbitrap resolution of 120000 , with the normalized AGC target of $300 \%$ (3E6) and max ion injection time of $25 \mathrm{~ms}$. Data-dependent acquisition (DDA) was performed for the top 15 precursor ions with the charge state of 2-6 and an isolated width of 2 . Intensity threshold was 5E3. Dynamic exclusion was $20 \mathrm{~s}$ with the exclusion of isotopes. Other settings for DDA include Orbitrap resolution of 15000 , HCD collision energy of $30 \%$, and ion injection time of $40 \mathrm{~ms}$.

Raw data files were analyzed by Andromeda Search engine incorporated in MaxQuant v1.6.17.0 software against human and E. Coli databases downloaded from Uniprot. Label-free quantitation was enabled in the analysis to compute $\mathrm{BAAQ}$ values for the identified proteins. 


\section{Negative-stain electron microscopy} continuous carbon-coated grids, blotted with filter paper, and stained with freshly prepared $0.75 \%$ microscope (FEI Company) equipped with a 4k x 4k CCD camera (UltraScan 4000, Gatan) and operated at a voltage of $120 \mathrm{kV}$. Images were recorded at room temperature with a nominal magnification of $52,000 \mathrm{x}$, corresponding to a calibrated pixel size of $2.21 \AA$ on the specimen. A

1015 from the micrographs and sorted through multiple rounds of 2D classification to discard poorly 1016 defined particle classes using Relion 3.0. A subset of 231,879 particles with well-defined shapes 1017 was selected following multiple rounds of 2D classification to demonstrate 2D class averages of 1018 COQ7-COQ9 complex.

\section{Cryo-EM sample preparation}

1021 Peak fractions containing the COQ7-COQ9 complex were concentrated to $5 \mathrm{mg} \mathrm{ml}^{-1}$ and flash

1022 frozen in liquid nitrogen for long-term storage at $-80^{\circ} \mathrm{C}$. An aliquot of purified COQ7-COQ9 1023 complex was diluted to $0.5 \mathrm{mg} \mathrm{ml}^{-1}(8.8 \mu \mathrm{M})$ in $200 \mathrm{mM}$ Ammonium Acetate, $\mathrm{pH} 7.0$ before 1024 blotting. Cryo-EM samples of COQ7-COQ9 complex bound to NADH were prepared similarly 
final NADH concentration of $5 \mathrm{mM}$ in a final solution containing 200mM Ammonium Acetate, $\mathrm{pH}$ 7.0, and incubated at room temperature for 1 hour. To prepare cryo-EM grids, $3.5 \mu 1$ of purified COQ7-COQ9 complex was applied to glow-discharged holey carbon grids (Quantifoil R1.2/1.3, 400 mesh $\mathrm{Cu}$ ) and incubated for an additional $30 \mathrm{~s}$. Then, the grids were blotted with Whatman Grade 1 filter paper (Whatman) for $\sim 6 \mathrm{~s}$ with a $0 \mathrm{~mm}$ offset at $10^{\circ} \mathrm{C}$ and $100 \%$ relative humidity and plunge frozen into liquid ethane using a Vitrobot Mark IV (Thermo Fisher Scientific).

\section{Data collection}

1034 Two datasets were collected on two different microscopes using the multi-record strategy (nine-

1035 hole exposures per single-stage movement) in SerialEM software ${ }^{10}$. For unliganded COQ7-COQ9

1036 complex, images were collected on a FEI Talos Arctica equipped with a K3 Summit direct electron

1037 detector (Gatan) and operated at an accelerating voltage of $200 \mathrm{keV}$. In total, 1,395 images were

1038 recorded with a defocus range of -0.5 to $-1.5 \mu \mathrm{m}$ in super-resolution counting mode at a nominal 1039 magnification of $36,000 x$, corresponding to a super-resolution pixel size of $0.57 \AA$ (physical pixel

1040 size of $1.14 \AA$ ) on the specimen level. Each image was dose-fractionated over 120 frames with a 1041 per frame dose rate of $\sim 0.49$ electrons and total exposure time of $2.4 \mathrm{~s}$, resulting in an accumulated 1042 dose of $\sim 58.8$ electrons per $\AA^{2}$. For NADH-bound COQ7-COQ9 complex, images were acquired 1043 on a FEI Titan Krios equipped with a K3 Summit direct electron detector and a Quantum GIF 1044 energy filter (Gatan) with a slit width of $20 \mathrm{eV}$ and operated an accelerating voltage of $300 \mathrm{keV}$ in 1045 nano-probe mode. A total of 10,088 micrographs were collected at a magnification of 105,000x 1046 with a calibrated super-resolution pixel size of $0.4165 \AA$ (physical pixel size of $0.833 \AA$ ) and a 1047 defocus range of -0.3 to $-1.2 \mu \mathrm{m}$. The total exposure time for each micrograph was $3 \mathrm{~s}$, with dose1048 fractionation set at $0.025 \mathrm{~s}$ per frame, resulting in 118 movie frames at a per frame dose rate of 
1049

1050

1051

1052

1053

1054

1055

1056

1057

1058

1059

1060

1061

1062

1063

1064

1065

1066

1067

1068

1069

1070

1071

$\sim 0.55$ electrons for a total accumulated dose of $\sim 65$ electrons per $\AA^{2}$. Data collection statistics are shown in Extended Data Table 1.

\section{Image analysis and $3 D$ reconstruction}

Dose-fractionated stacks were subjected to beam-induced motion correction using MotionCor $2^{11}$. CTF parameters for each micrograph in the unliganded COQ7-COQ9 complex and the NADHbound COQ7-COQ9 complex datasets were determined by GCTF v.1.06 ${ }^{12}$ and CTFFIND4 ${ }^{8}$, respectively. Subsequent image processing for both datasets was carried out in Relion 3.0. For cofactor-free COQ7-COQ9 complex, 3040 particles were manually picked and classified by reference-free 2D classification to generate templates for automatic particle picking. A total of 574,760 auto-picked particles were extracted with a box size of 192 pixels and subjected to two rounds of 2D classification to discard poorly defined classes, resulting in 528,971 particles for further processing. An initial 3D model from these particles was generated by using cryoSPARC $a b$ initio reconstruction ${ }^{13}$. Stable classes were then used for iterative rounds of $3 \mathrm{D}$ refinement and reclassification without symmetry. Upon visual inspection of $3 \mathrm{D}$ reconstructions in UCSF Chimera $^{3}$, the particles from the best 3D classes $(281,866$ particles) were combined and subjected

to 3D refinement with D2 symmetry. Subsequent per-particle CTF refinement and 3D classification without alignment yielded a single class containing 61,526 particles. This final subset of particles was refined with a soft mask and sharpened by a negative temperature factor to a resolution of $3.5 \AA$ during post-processing procedure. A similar strategy was used for NADHbound COQ7-COQ9 complex. In total, 2,709,398 particles were extracted with a box size of 256 pixels and binned to 128 pixels. After removing the suboptimal particles with two rounds of reference-free $2 \mathrm{D}$ classification, the best classes were subjected to $3 \mathrm{D}$ refinement without imposed 
1072 symmetry. Subsequent 3D classification into 6 classes showed two dominant classes containing

$10731,249,119$ particles ( $46 \%$ of the dataset), which was then re-extracted to a box size of 256 pixels

1074 and refined with D2 symmetry imposed. These particles were subjected to another round of 3D

1075 classification into six classes without image alignment, resulting in 372,917 particles with

1076 indicated global resolution of $2.7 \AA$. The map was further improved after CTF and aberration

1077 refinements by using Relion $3.1^{9}$. The final map had a resolution of $2.4 \AA$ after sharpened by

1078 applying a negative temperature factor during the post-processing step. The reported final

1079 resolution estimates are based on the gold-standard Fourier shell correlation (FSC) cut-off of

1080 0.143. Local resolutions were determined using ResMap with half-reconstructions as input maps.

1081 Refinement parameters for the final density maps are listed in Extended Data Table 1.

\section{Model building and validation}

1084 The initial model of COQ7 was a homology model calculated by Phyre $2^{14}$, using the intensive 1085 modeling mode. The crystal structure of COQ9 (PDB ID: 6AWL) was also used as an initial 1086 template for model building. The atomic models were manually rebuilt using $\operatorname{Coot}^{15}$ and real-space 1087 refined using phenix.real_space_refine too ${ }^{16}$ from the PHENIX software package ${ }^{17}$. Briefly, 1088 phenix.mtriage ${ }^{17}$ was used to analyze cryo-EM maps and an automated sharpening procedure 1089 (phenix.auto_sharpen ${ }^{10}$ ) was applied to the final cryo-EM reconstructions prior to model building. 1090 The $3.7 \AA$ and $2.4 \AA$ density maps were of sufficient quality for de novo model building. After 1091 initial model building, the unliganded and NADH-bound starting models were subjected to 1092 iterative rounds of refinement in phenix.real_space_refine using global minimization, morphing,

1093 noncrystallographic symmetry (NCS), B-factor refinement, local grid search and secondary 1094 structure restraints, and manual rebuilding in Coot. The ligand coordinates were docked into 
1095 densities and refined using Coot. The final model geometry was evaluated using MolProbity $4.5^{18}$.

1096 Independent FSC curves for model-map correlations were calculated between the resulting model

1097 and the half map used for refinement as well as between the resulting model and the other half

1098 map for cross-validation. Protein interfaces and associated free energies were analyzed by using

1099 PDBePISA server ${ }^{19}$. The final refinement statistics are summarized in Extended Data Table 1.

1101 Molecular dynamics simulations

1102 We prepared all systems for MD simulations with the membrane module of the CHARMM-GUI

1103 server ${ }^{20}$ and followed the provided equilibration and production files, except for changes as

1104 indicated.

1105 We parametrized the protein components with the CHARMM36m forcefield, its adapted TIP3P

1106 water model and the CHARMM36 lipids ${ }^{21}$, plus the NADH parameters from ${ }^{22}$ and the CoQ 10

1107 parameters from ${ }^{23,24}$. The model membranes contained 17\% cardiolipin (charged -2), 44\%

1108 POPC and 39\% POPE; or 16\% cardiolipin, 42\% POPC and 38\% POPE when 4\% CoQ10 was

1109 included. The solvent included $0.15 \mathrm{M} \mathrm{KCl}$ for charge neutralization and ionic strength.

1110 For simulations aimed at testing unbinding of CoQ10 from COQ7's active site, we first tried

1111 replica exchange MD but it invariably resulted in membrane destabilization. Therefore, we took

1112 another approach in which we ran regular simulations at 300, 400, 450, 500 and $600 \mathrm{~K}$. At 500

1113 and $600 \mathrm{~K}$, the system destabilized within the first few hundred nanoseconds of simulation times:

1114 the proteins unfolded and the membranes disrupted. At 300 and $400 \mathrm{~K}$, the small molecule

1115 remained locked inside the active site for the full length of independent multi-microsecond

1116 trajectories. At $450 \mathrm{~K}$ we observed events in which the docked CoQ10 dissociated from the

1117 active site without compromising the protein or membrane stabilities within the simulated 
1118 timescale ( 1-1.5 microsecond); we therefore analyzed 10 independent replicas at this

1119 temperature as described in the main text. In all these simulations the membranes and proteins

1120 remained stable, and the NADH remained stably bound to COQ7, during the whole simulation

1121 times.

1122

1123 We ran all simulations with Gromacs $2020^{25}$, visualized them with $\mathrm{VMD}^{26}$ and analyzed them

1124 with custom scripts, VMD procedures, and the MEMBPLUGIN plugin for VMD ${ }^{27}$.

1125

1126

Data and Code Availability

1127 The raw LC-MS lipidopmics data generated during the current study are available from the

1128 corresponding author on reasonable request.

1130 The atomic coordinates of NADH-bound COQ7:COQ9 complex and COQ7:COQ9 complex only

1131 have been deposited in the Protein Data Bank with accession numbers 7SSS and 7SSP,

1132 respectively. All of the 3D cryoEM density maps associated with this study have been deposited

1133 in the Electron Microscopy Data Bank under accession numbers EMD-25413 for NADH-bound

1134 COQ7:COQ9 complex and EMD-25412 for COQ7:COQ9 complex only.

1136 No custom computer code was generated for this project.

\section{Methods References}

1139 1. Gietz, R.D. \& Woods, R.A. Transformation of yeast by lithium acetate/single-stranded $1140 \quad$ carrier DNA/polyethylene glycol method. Methods in Enzymology 350, 87-96 (2002).

1141 2. Pravda, L. et al. MOLEonline: a web-based tool for analyzing channels, tunnels and $1142 \quad$ pores (2018 update). Nucleic Acids Research 46, W368-W373 (2018). 
1143 3. Pettersen, E.F. et al. UCSF Chimera-A visualization system for exploratory research

1144

1145

1146

1147

1148

1149

1150

1151

1152

1153

1154

1155

1156

1157

1158

1159

1160

1161

1162

1163

1164

1165

1166

1167

1168

1169

1170

1171

1172

1173

1174

1175

1176

1177

1178

1179

1180

1181

1182

1183

1184

1185

1186

1187

1188 and analysis. Journal of Computational Chemistry 25, 1605-1612 (2004).

4. Jurrus, E. et al. Improvements to the APBS biomolecular solvation software suite. Protein Science 27, 112-128 (2018).

5. Schneider, C.A., Rasband, W.S. \& Eliceiri, K.W. NIH Image to ImageJ: 25 years of image analysis. Nature Methods 9, 671-675 (2012).

6. Matyash, V., Liebisch, G., Kurzchalia, T.V., Shevchenko, A. \& Schwudke, D. Lipid extraction by methyl-tert-butyl ether for high-throughput lipidomics. J Lipid Res 49, 1137-46 (2008).

7. Hutchins, P.D., Russell, J.D. \& Coon, J.J. LipiDex: An Integrated Software Package for High-Confidence Lipid Identification. Cell Syst 6, 621-625 e5 (2018).

8. Rohou, A. \& Grigorieff, N. CTFFIND4: Fast and accurate defocus estimation from electron micrographs. Journal of Structural Biology 192, 216-221 (2015).

9. Zivanov, J. et al. New tools for automated high-resolution cryo-EM structure determination in RELION-3. eLife 7, e42166 (2018).

10. Mastronarde, D.N. Automated electron microscope tomography using robust prediction of specimen movements. Journal of Structural Biology 152, 36-51 (2005).

11. Zheng, S.Q. et al. MotionCor2: anisotropic correction of beam-induced motion for improved cryo-electron microscopy. Nature Methods 14, 331-332 (2017).

12. Zhang, K. Gctf: Real-time CTF determination and correction. Journal of Structural Biology 193, 1-12 (2016).

13. Punjani, A., Rubinstein, J.L., Fleet, D.J. \& Brubaker, M.A. cryoSPARC: algorithms for rapid unsupervised cryo-EM structure determination. Nature Methods 14, 290-296 (2017).

14. Kelley, L.A., Mezulis, S., Yates, C.M., Wass, M.N. \& Sternberg, M.J.E. The Phyre2 web portal for protein modeling, prediction and analysis. Nature Protocols 10, 845-858 (2015).

15. Emsley, P., Lohkamp, B., Scott, W.G. \& Cowtan, K. Features and development of Coot. Acta Crystallographica Section D: Biological Crystallography 66, 486-501 (2010).

16. Afonine, P.V. et al. Real-space refinement in PHENIX for cryo-EM and crystallography. Acta Crystallographica. Section D, Structural Biology 74, 531-544 (2018).

17. Liebschner, D. et al. Macromolecular structure determination using X-rays, neutrons and electrons: recent developments in Phenix. Acta Crystallographica. Section D, Structural Biology 75, 861-877 (2019).

18. Williams, C.J. et al. MolProbity: More and better reference data for improved all-atom structure validation. Protein Science 27, 293-315 (2018).

19. Krissinel, E. \& Henrick, K. Inference of Macromolecular Assemblies from Crystalline State. Journal of Molecular Biology 372, 774-797 (2007).

20. Lee, J. et al. CHARMM-GUI Membrane Builder for Complex Biological Membrane Simulations with Glycolipids and Lipoglycans. Journal of Chemical Theory and Computation 15, 775-786 (2018).

21. Huang, J. et al. CHARMM36m: an improved force field for folded and intrinsically disordered proteins. Nature Methods 14, 71-73 (2017).

22. Pavelites, J.J., Gao, J., Bash, P.A. \& Mackerell, A.D. A molecular mechanics force field for NAD+ NADH, and the pyrophosphate groups of nucleotides. Journal of Computational Chemistry 18, 221-239 (1997). 
23. Teixeira, M.H. \& Arantes, G.M. Effects of lipid composition on membrane distribution and permeability of natural quinones. RSC Advances 9, 16892-16899 (2019).

24. Galassi, V.V. \& Arantes, G.M. Partition, orientation and mobility of ubiquinones in a lipid bilayer. Biochimica et Biophysica Acta (BBA) - Bioenergetics 1847, 1560-1573 (2015).

25. Abraham, M.J. et al. GROMACS: High performance molecular simulations through multi-level parallelism from laptops to supercomputers. SoftwareX 1, 19-25 (2015).

26. Humphrey, W., Dalke, A. \& Schulten, K. VMD: Visual molecular dynamics. Journal of Molecular Graphics 14, 33-38 (1996).

27. Guixà-González, R. et al. MEMBPLUGIN: studying membrane complexity in VMD. Bioinformatics 30, 1478-1480 (2014).

\section{Acknowledgments}

We thank current and former members of the D.J.P. and A.F. laboratories for helpful discussions and assistance on this project. We specially thank Danielle C. Lohman for pioneering the purification of the COQ7:COQ9 complex that paved the way for its experimental characterization. We thank Annie Jen and Zixiang Fang for their help with LC-MS measurements. We also thank Professor Stephen J. Lippard for kindly providing the pET30 GB1-Nd38_COQ7 vector. M. Braunfeld, D. Bulkley, M. Harrington, A. Myasnikov, and Z. Yu of the UCSF Center for Advanced CryoEM for microscopy support and J. Baker-LePain and the QB3 shared cluster (NIH grants 1S10OD021596-01, S10OD020054, S10OD026881, 1S10OD021741 and the Howard Hughes Medical Institute).

\section{This work was supported by NIH awards R35 GM131795 (D.J.P.), P41 GM108538 (J.J.C. and} D.J.P.), support from the Howard Hughes Medical Institute Faculty Scholar program and the Chan Zuckerberg Biohub (A.F.); funds from the BJC Investigator Program (D.J.P.); SNSF grants 205321_192371 and 31003A_170154 (M.D.P). 
1218 H.A. is a Human Frontier Science Program Long-Term Fellow supported by The International

1219 Human Frontier Science Program Organization (LT000398/2017-L).

\section{Author contributions}

1222 Mateusz Manicki: Conceptualization, Methodology, Formal analysis, Investigation, Writing 1223 original draft, Writing - review and editing, Visualization, Project Administration. Halil Aydin:

1224 Conceptualization, Methodology, Software, Formal analysis, Investigation, Data curation,

1225 Writing - review and editing. Luciano A. Abriata: Conceptualization, Methodology, Software, 1226 Formal analysis, Investigation, Data curation, Writing - review and editing, Visualization.

1227 Katherine A. Overmyer: Methodology, Formal analysis, Investigation, Data curation. Rachel

1228 M. Guerra: Conceptualization, Methodology, Writing - review and editing. Joshua J. Coon:

1229 Supervision, Funding acquisition. Matteo Dal Peraro: Methodology, Writing - review and

1230 editing, Supervision, Funding acquisition. Adam Frost: Conceptualization, Methodology,

1231 Writing - review and editing, Supervision, Funding acquisition. David J. Pagliarini:

1232 Conceptualization, Methodology, Writing - review and editing, Supervision, Project

1233 administration, Funding acquisition.

1234

1235 Competing interests

1236 J.J.C. is a consultant for Thermo Scientific.

1237 A.F. is a shareholder and consultant for Relay Therapeutics, LLC.

1239 Correspondence and requests for materials

1240 Requests should be addressed to A.F. or D.J.P. 
bioRxiv preprint doi: https://doi.org/10.1101/2021.11.15.468694; this version posted November 15,2021 . The copyright holder for this preprint (which was not certified by peer review) is the author/funder, who has granted bioRxiv a license to display the preprint in perpetuity. It is made available under aCC-BY-NC-ND 4.0 International license.

1241 Figure 1

1242

1243

1244

1245

1246

1247

1248

1249

1250

1251

1252

1253

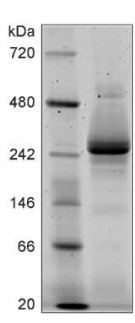

b



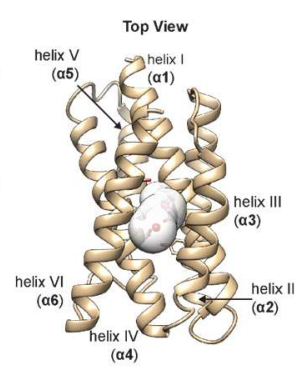

(a4)

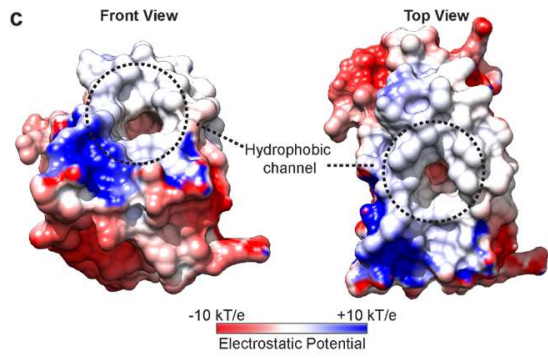

d

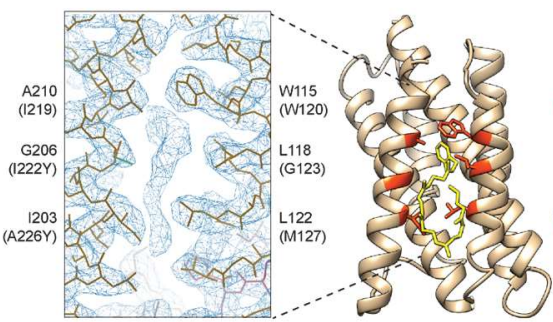

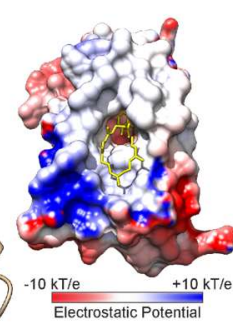
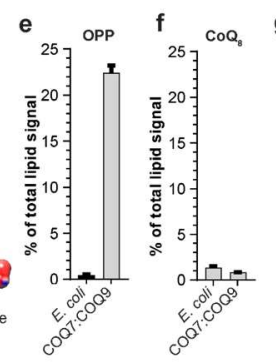

$$
\text { g }
$$
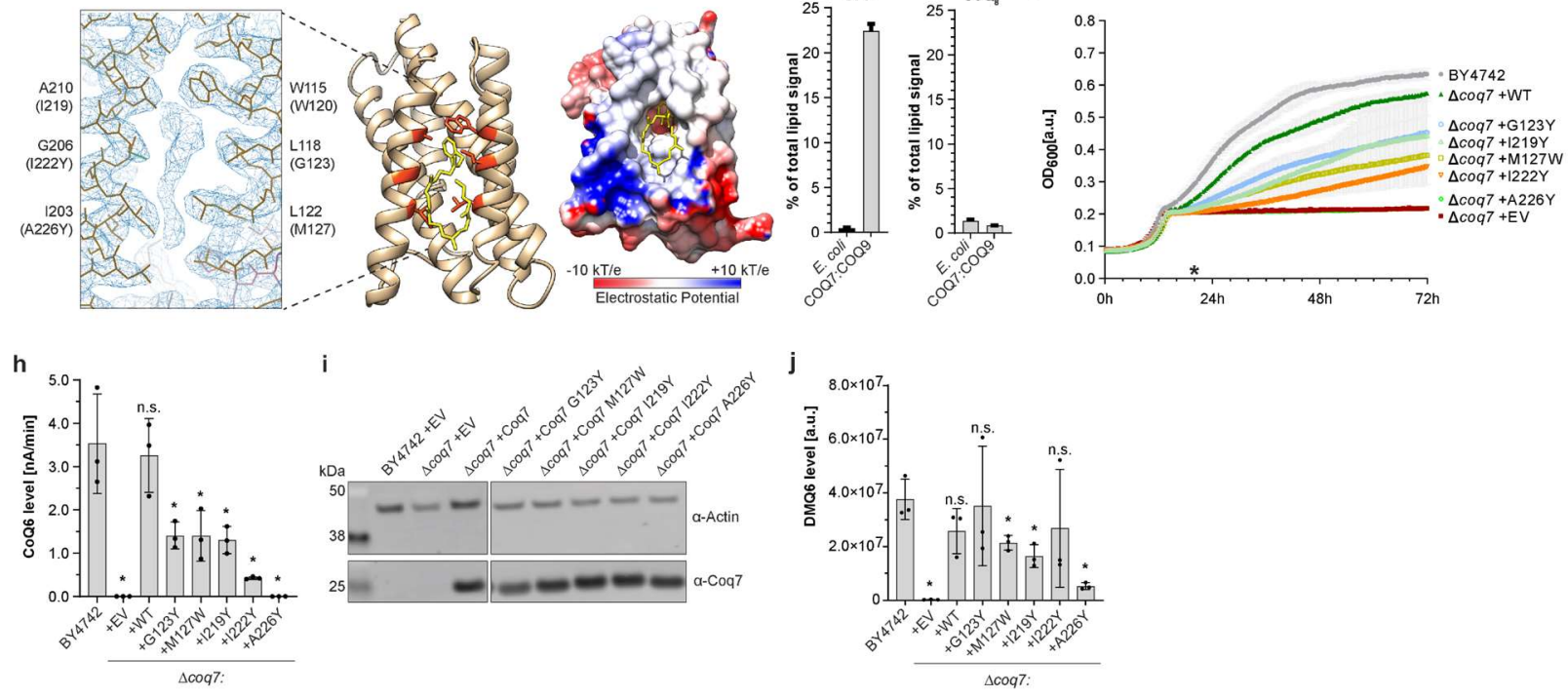

1254

1255

1256

1257

1258

1259

1260

1261

1262

1263

1264 


\section{Figure 1. Structure - function characterization of COQ7.}

1266

1267

1268

1269

1270

1271

1272

1273

1274

1275

1276

1277

1278

1279

1280

1281

1282

1283

1284

1285

1286

1287

a, Native PAGE analysis of the homogeneity and size of purified GB1- ${ }^{\mathrm{Nd} 38} \mathrm{COQ} 7:$ His6${ }^{\mathrm{Nd} 79}$ COQ9 complex. b, Structure of COQ7 with visible channel leading to an active site. Iron atoms were not resolved by cryoEM and were added based on the structure of bactoferritin (PDB:4AM2). c, Electrostatic surface potential of COQ7 with visible entry to the hydrophobic channel. d, Detailed view of the hydrophobic channel with visible additional ligand density inside. Important residues are labeled. Corresponding residues in yeast are in parentheses. e,f, LC-MS lipidomic analysis of relative levels of Octaprenylphenol (OPP) and Coenzyme $\mathrm{Q}_{8}$ $\left(\mathrm{CoQ}_{8}\right)$ in $E$. coli cells and the GB1- ${ }^{\mathrm{Nd} 38} \mathrm{COQ} 7: \mathrm{His}^{-}{ }^{\mathrm{Nd} 79} \mathrm{COQ} 9$ complex purified from E. coli. (mean \pm s.d., $n=2$ ) g, Respiratory growth of wild type BY4742 yeast and Coq7 mutants expressed in BY4742 $\Delta$ coq7 genetic background. Cells start with fermentation and then switch to respiratory growth (mean \pm s.d., $n=3$ ). The star $(*)$ corresponds to time point used for measurements in panel h,i,j. h, HPLC-ECD analysis of Coenzyme Q6 level in wild type BY4742 yeast and Coq7 mutants expressed in BY4742 $\Delta$ coq7 genetic background. Signal normalized to internal CoQ 8 control (mean \pm s.d., $n=3$, one-sided Student's $t$-test, ${ }^{*} \mathrm{p}<0.05$, n.s. not significant). i, Expression level of the Coq7 yeast mutants (p413, TEF promoter) measured with Western Blot. Native Coq7 in BY4742 strain is below the limit of detection. Samples were prepared in triplicates. Representative membrane shown. j) LC-MS lipidomic analysis of DMQ 6 substrate level in wild type BY4742 yeast and Coq7 mutants expressed in BY4742 $\Delta$ coq7 genetic background. Signal normalized to internal $\mathrm{CoQ}_{8}$ control (mean \pm s.d., $n=3$, one-sided Student's $t$-test, ${ }^{*} \mathrm{p}<0.05$, n.s. not significant). 
bioRxiv preprint doi: $h t t p s: / / d o i . o r g / 10.1101 / 2021.11$ 15.468694: this version posted November 15,2021 . The copyright holder for this preprint (which was not certified by peer review) is the author/funder, who has granted bioRxiv a license to display the preprint in perpetuity. It is made available under aCC-BY-NC-ND 4.0 International license.

Figure 2

1289

1290

1291

1292

1293

1294

1295

1296

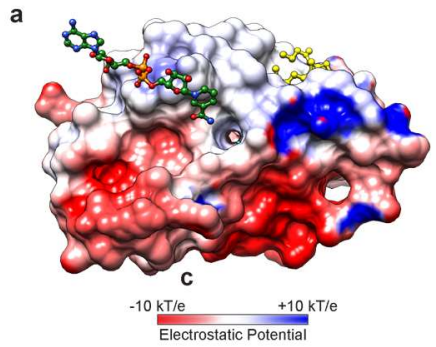

c



1297

1298

1299

1300

1301

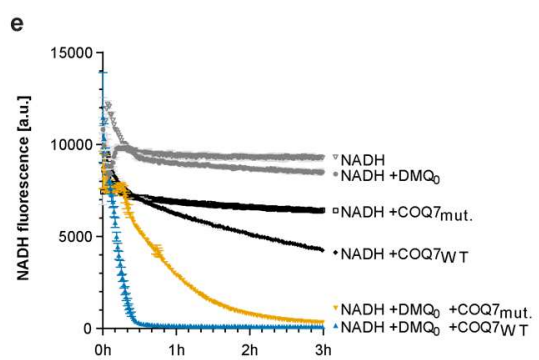


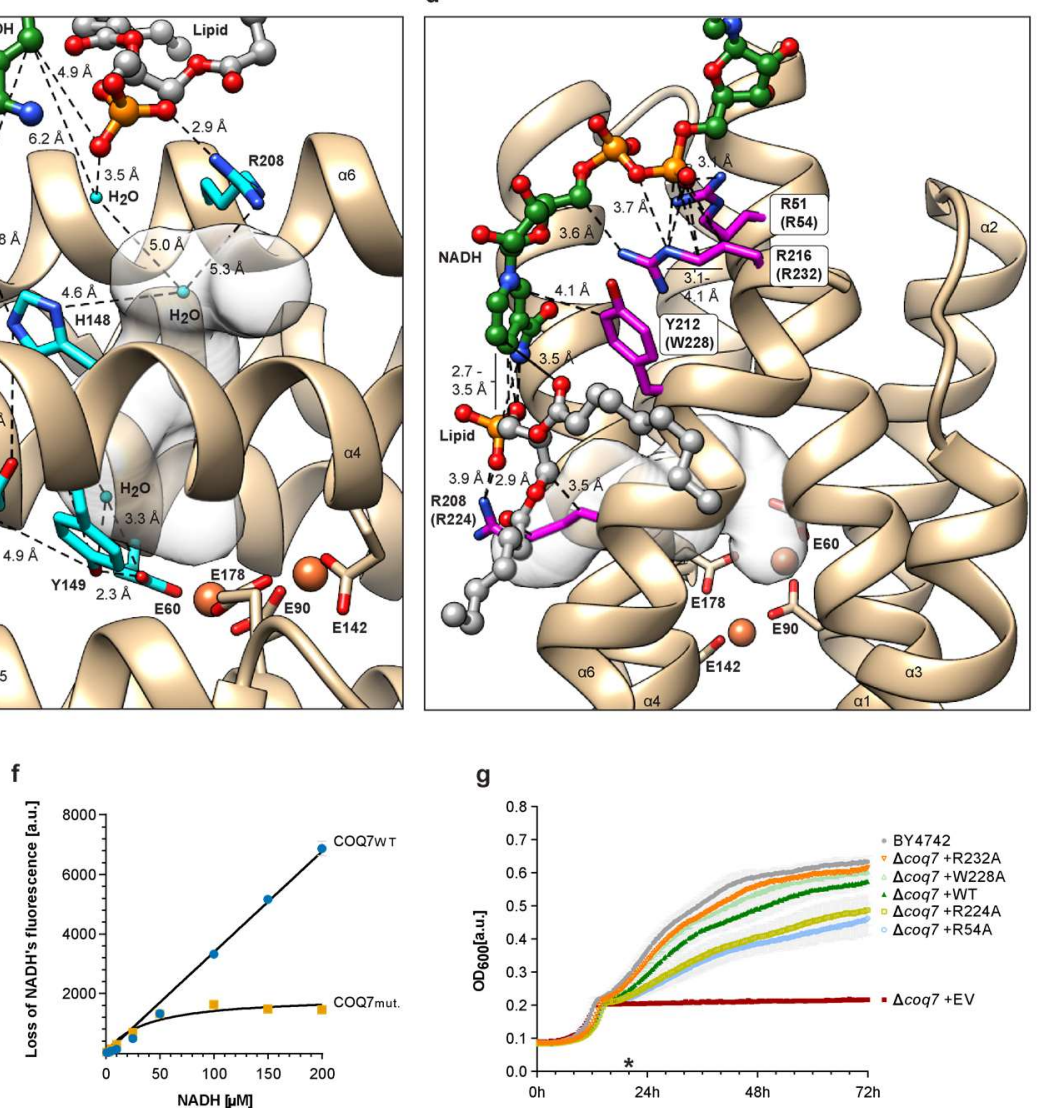

1302

1303

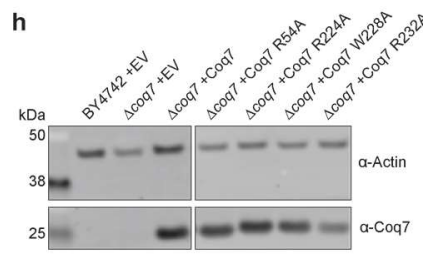

1304

1305

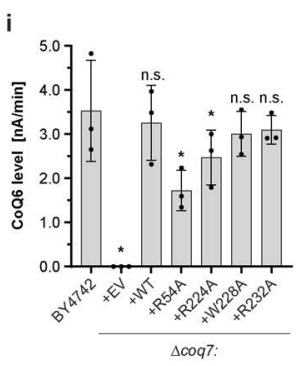




\section{Figure 2. Details of NADH binding to COQ7.}

1312

1313

1314

1315

1316

1317

1318

1319

1320

1321

1322

1323

1324

1325

1326

1327

1328

1329

1330

1331

1332

1333

a, Electrostatic surface potential of COQ7 with bound NADH molecule (green) and OPP

(yellow). b, Ribbon view of COQ7's NADH binding site and channel leading to the active site.

c, Structural elements that might participate in charge transfer from NADH to COQ7. d, Details

of NADH binding by conserved arginines and tyrosine. e, NADH oxidation in vitro assay

showing activities of WT and RRYR/AAAA quad COQ7 mutant (mean \pm s.d., $n=3$ ). f, Effect of

the RRYR/AAAA mutation as a function of NADH concentration. The graph shows 30 min.

time point extracted from data presented in Extended Data. Fig. 9g,h (mean \pm s.d., $n=3$ ). g,

Respiratory growth of wild type BY4742 yeast and Coq7 mutants expressed in BY4742 $\Delta$ coq7

genetic background. Cells start with fermentation and then switch to respiratory growth (mean \pm

s.d., $n=3$ ). The star $(*)$ corresponds to time point used for measurements in panel h,i, $\mathbf{h}$,

Expression level of the Coq7 yeast mutants (TEF promoter) measured with Western Blot. Native

Coq7 in BY4742 strain is below the limit of detection. Samples were prepared in triplicates.

Representative membrane shown. i, HPLC-ECD analysis of Coenzyme Q6 $\left(\mathrm{CoQ}_{6}\right)$ level in wild type BY4742 yeast and Coq7 mutants expressed in BY4742 $\Delta$ coq7 genetic background. Signal normalized to internal $\mathrm{CoQ}_{8}$ control (mean \pm s.d., $n=3$, one-sided Student's $t$-test, ${ }^{*} \mathrm{p}<0.05$, n.s. not significant). 
Figure 3
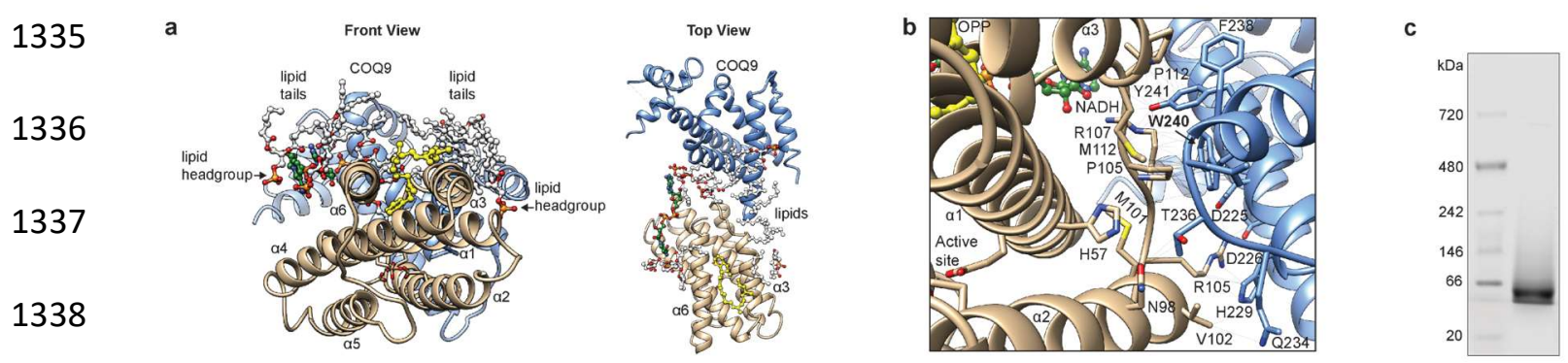

1339 d

1340

1341

1342
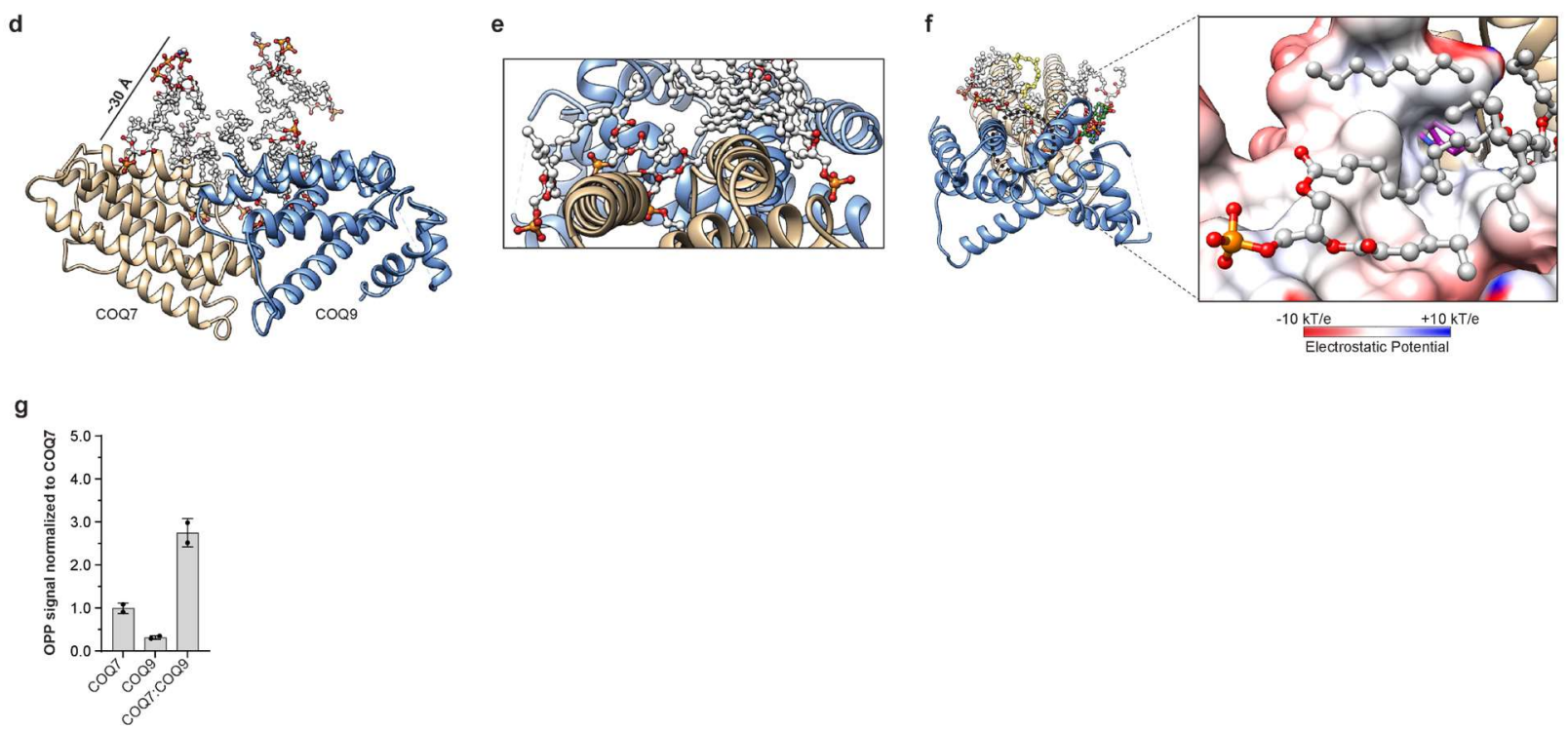

Figure 3. Structure of COQ7:COQ9 heterodimer in lipid environment.

a, Snapshot of the COQ7:COQ9 heterodimer structure with visible lipids (light gray), NADH the mutated GB1- ${ }^{\mathrm{Nd} 38} \mathrm{COQ} 7: H i s 6-{ }^{\mathrm{Nd} 79} \mathrm{COQ} 9{ }^{\mathrm{W} 240 \mathrm{~K}}$ complex. d, Zoom on lipid molecules forming binding substrate pocket in COQ9 surrounded by surface lipids. No quinone density is detected 
a

1359

1360

1361

1362

1363

1364

1365

1366

1367

1368

1369

1370

1371

1372

1373

1374

1375

1376

1377

1378
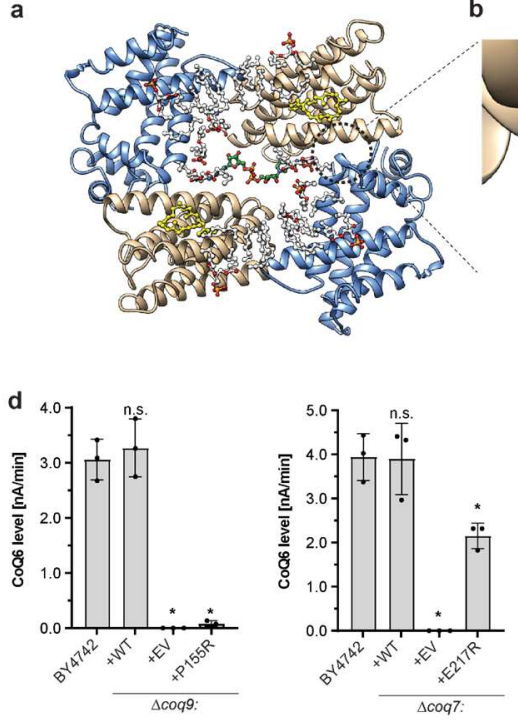

c




e

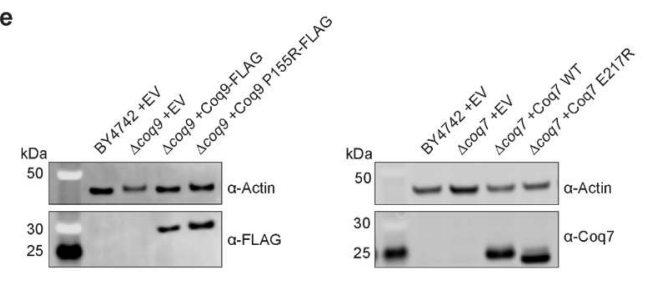

Figure 4. Structure of the COQ7:COQ9 heterotetramer.

a, Membrane view of an individual COQ7:COQ9 tetramer. b, Details of COQ7:COQ9

interaction mediating tetramer's formation. COQ9 P210 and COQ7 S201 residues are labeled in bold and their corresponding residues in yeast are provided in parentheses. c, Respiratory growth of Coq7 or Coq7 mutants expressed in BY4742 $\Delta \operatorname{coq} 7 / \Delta \operatorname{coq} 9$ genetic background. Cells start with fermentation and then switch to respiratory growth (mean \pm s.d., $n=3$ ). The star $(*)$ corresponds to time point used for measurements in panel d,e. d, HPLC-ECD analysis of Coenzyme Q6 level in wild type BY4742 yeast and Coq7 or Coq9 mutant expressed in BY4742 $\Delta \operatorname{coq} 7$ or $\Delta \operatorname{coq} 9$ genetic background. Signal normalized to internal $\mathrm{CoQ}_{8}$ control (mean \pm s.d., $n$ $=3$, one-sided Student's $t$-test, ${ }^{*} \mathrm{p}<0.05$, n.s. not significant) e, Expression level of the Coq 7 yeast mutants (TEF promoter) measured with Western Blot. Native Coq7 in BY4742 strain is below the limit of detection. Samples were prepared in triplicates. Representative membrane 1379 shown. 
bioRxiv preprint doi: $h t t p s: / / d o i . o r g / 10.1101 / 2021.11 .15 .468694$; this version posted November 15,2021 . The copyright holder for this preprint (which was not certified by peer review) is the author/funder, who has granted bioRxiv a license to display the preprint in perpetuity. It is made available under aCC-BY-NC-ND 4.0 International license.

Figure 5
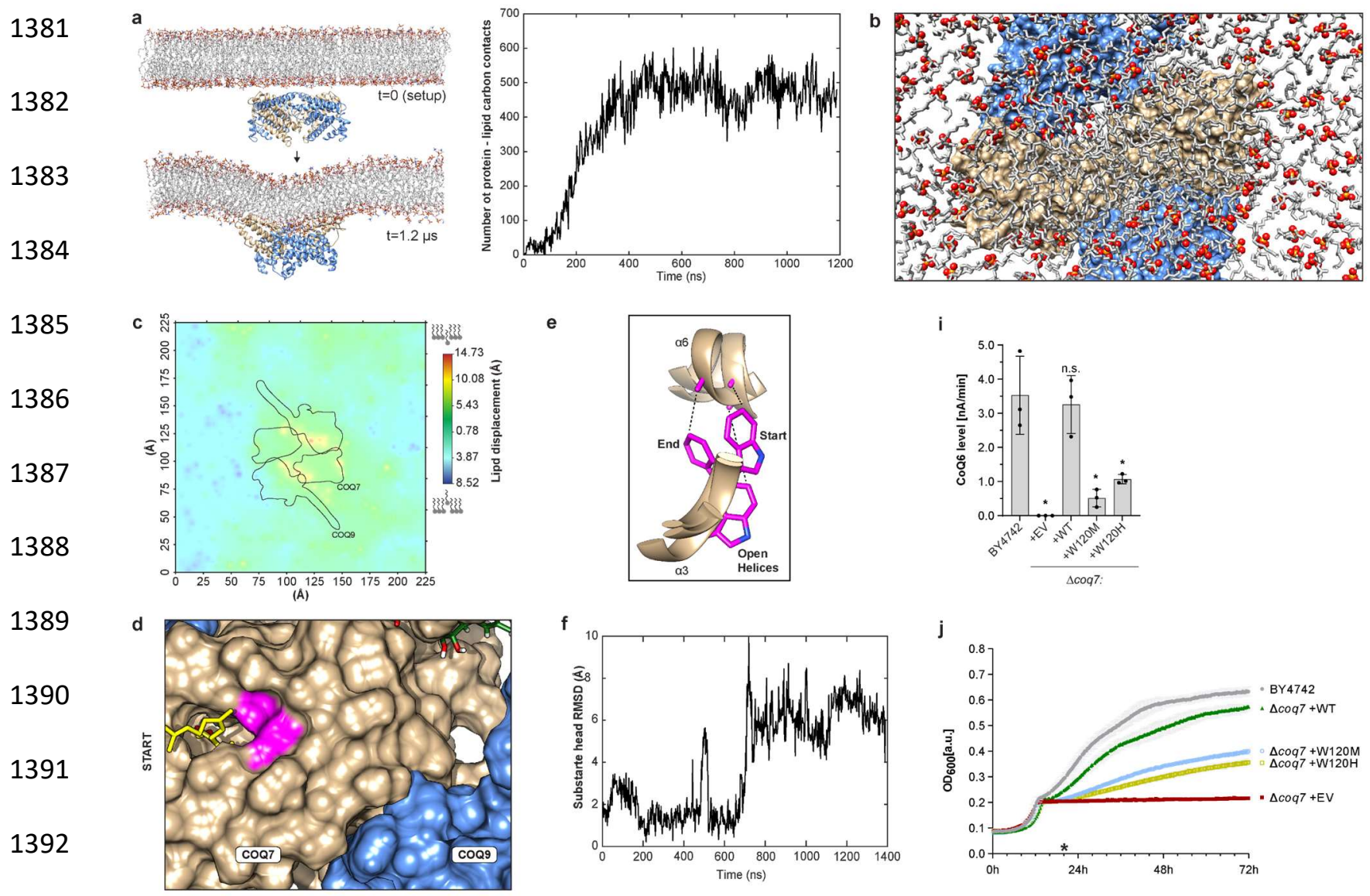

1393

1394

1395

1396

1397

1398

1399
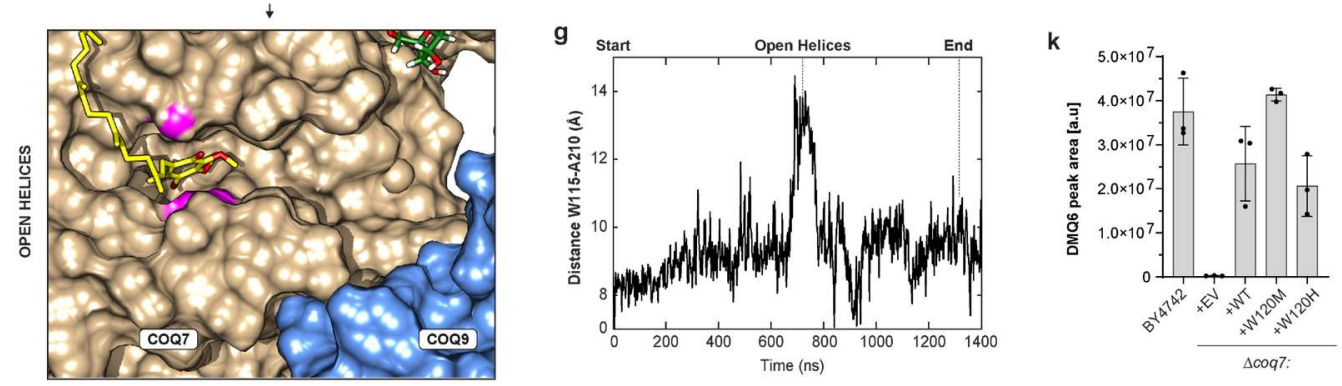

1400
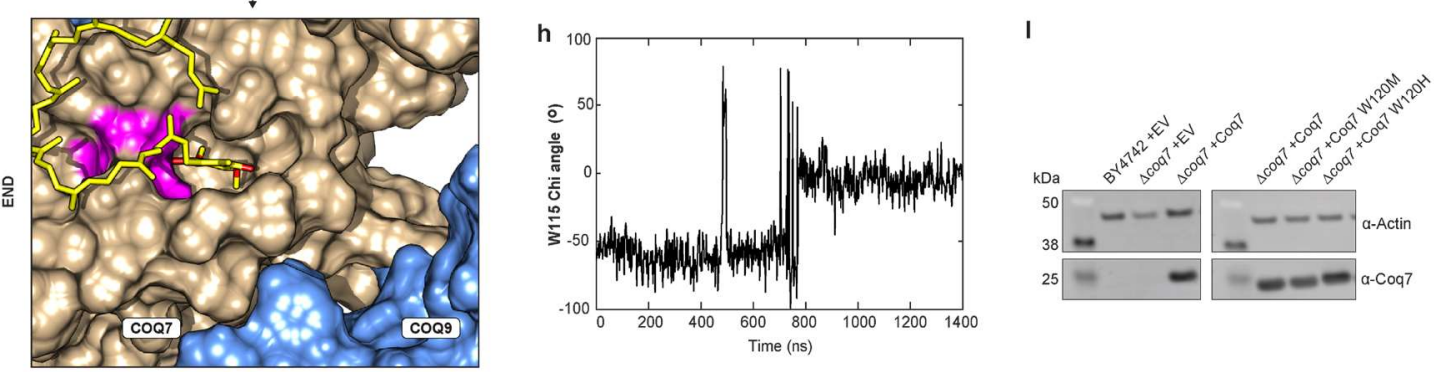

1401 


\section{Figure 5. Molecular dynamics simulations of the COQ7:COQ9 tetramer at the membrane.}

1404

a, Membrane binding and deformation observed in the atomistic MD simulations. The graph describes the number of contacts between COQ7 atoms and carbon atoms of all lipid tails. b, Shot of the protein-membrane complex observed after a few hundreds of ns of simulation when the tetramer has anchored to the membrane, exemplified here with the end point of one trajectory. Phosphate groups representing lipid headgroups are displayed as red spheres. Other atoms are displayed as sticks. COQ7 (tan) and COQ9 (blue) are visible as surfaces. Notice how COQ7 gains direct access to the hydrophobic portion of the membrane. c, Map of the deformation of the bottom membrane leaflet induced by the proteins binding to it. d, Snapshots from molecular dynamics simulation at $450 \mathrm{~K}$ showing release of CoQ 10 from COQ7 (tan) towards COQ9 (blue) by gating through W115 and A210 (magenta). e, RMSD of the CoQ10 molecule as a proxy for its displacement out of the active site. f, Overlaid positions of the gating W115 and A210 residues from COQ7 conformations visible in panel d. g, Time plot showing the distance between the $\mathrm{C} \alpha$ atoms of $\mathrm{W} 115$ and $\mathrm{A} 210$. $\mathbf{h}$, Time plot showing the sidechain torsion angle of W115. i) LC-MS lipidomic analysis of $\mathrm{DMQ}_{6}$ level in wild type BY4742 yeast and Coq7 mutants expressed in BY4742 $\Delta$ coq7 genetic background. Signal normalized to internal CoQ8 control (mean \pm s.d., $n=3$, one-sided Student's $t$-test, ${ }^{*} \mathrm{p}<0.05$, n.s. not significant). $\mathbf{j}$ HPLC-ECD analysis of Coenzyme $\mathrm{Q}_{6}\left(\mathrm{CoQ}_{6}\right)$ level in wild type BY4742 yeast and Coq7 mutants expressed in BY4742 $\Delta$ coq7 genetic background. Signal normalized to internal $\mathrm{CoQ}_{8}$ control (mean \pm s.d., $n=3$, one-sided Student's $t$-test, ${ }^{*} \mathrm{p}<0.05$, n.s. not significant). $\mathbf{k}$, Respiratory growth of wild type BY4742 yeast and Coq7 mutants expressed in BY4742 $\Delta$ coq7 genetic background. Cells start with fermentation and then switch to respiratory growth (mean \pm s.d., $n=3$ ). The star $(*)$ corresponds to time point used for measurements in panels $\mathrm{i}, \mathrm{j}, 1 . \mathbf{l} \mathbf{l}$, 
1426 Expression level of the Coq7 yeast mutants (TEF promoter) measured with Western Blot. Native

1427 Coq7 in BY4742 strain is below the limit of detection. Samples were prepared in triplicates.

1428 Representative membrane shown.

1429

1430

1431

1432

1433

1434

1435

1436

1437

1438

1439

1440

1441

1442

1443

1444

1445

1446

1447

1448 
a

1451

1452

1453

1454

1455

1456

1457

1458

1459

1460

1461

1462

1463

1464

1465

1466

1467

1468

1469

1470

1471
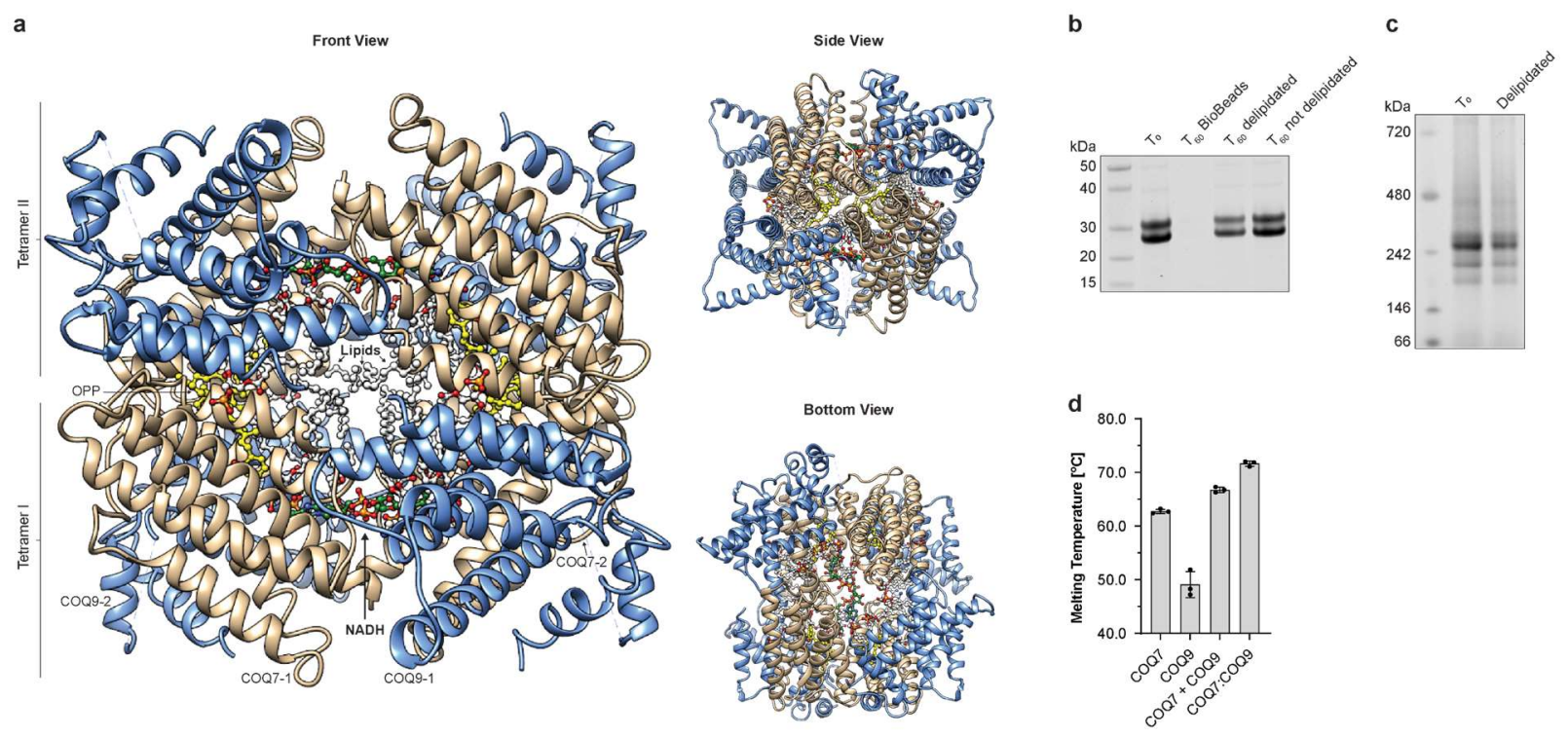

Figure 6. Two COQ7:COQ9 tetramers form an octamer.

a, Structure of the octamer with visible bound NADH (green) and OPP (yellow) b, SDS-PAGE analysis of protein stability upon delipidation of the GB1- ${ }^{\mathrm{Nd} 38} \mathrm{COQ7}: \mathrm{His6}-{ }^{\mathrm{Nd} 79} \mathrm{COQ} 9$ with BioBeads; $\mathrm{T}_{0}-$ soluble fraction before delipidation, $\mathrm{T}_{60}$ BioBeads - protein bound to beads after 60 min. incubation, $\mathrm{T}_{60}$ delipidated - soluble fraction after 60 min. delipidation, $\mathrm{T}_{60}$ not delipidated - soluble fraction after 60 min. incubation without BioBeads. c, Native PAGE analysis of the GB1- ${ }^{\mathrm{Nd} 38} \mathrm{COQ} 7: \mathrm{His} 6-{ }^{\mathrm{Nd} 79} \mathrm{COQ} 9$ complex after $15 \mathrm{~min}$. delipidation. d, DSF analysis of melting temperatures of individually purified His6- ${ }^{\mathrm{Nd} 38} \mathrm{COQ} 7$, His6- ${ }^{\mathrm{Nd} 79} \mathrm{COQ} 9$, their mix and the GB1- ${ }^{\mathrm{Nd} 38} \mathrm{COQ} 7:$ His6- ${ }^{\mathrm{Nd} 79} \mathrm{COQ} 9$ complex. 
bioRxiv preprint doi: https://doi.org/10.1101/2021.11.15.468694; this version posted November 15, 2021. The copyright holder for this preprint (which was not certified by peer review) is the author/funder, who has granted bioRxiv a license to display the preprint in perpetuity. It is made available under aCC-BY-NC-ND 4.0 International license.

Extended Data Figure 1

1473

1474

1475

1476

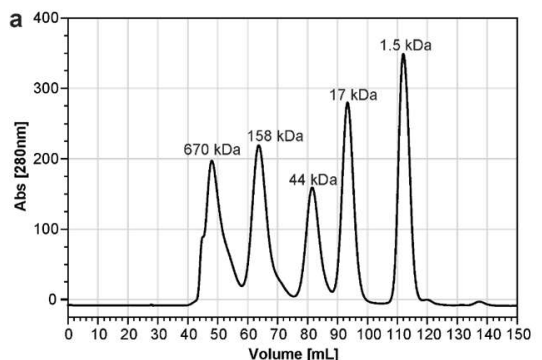

1477

1478

1479

1480
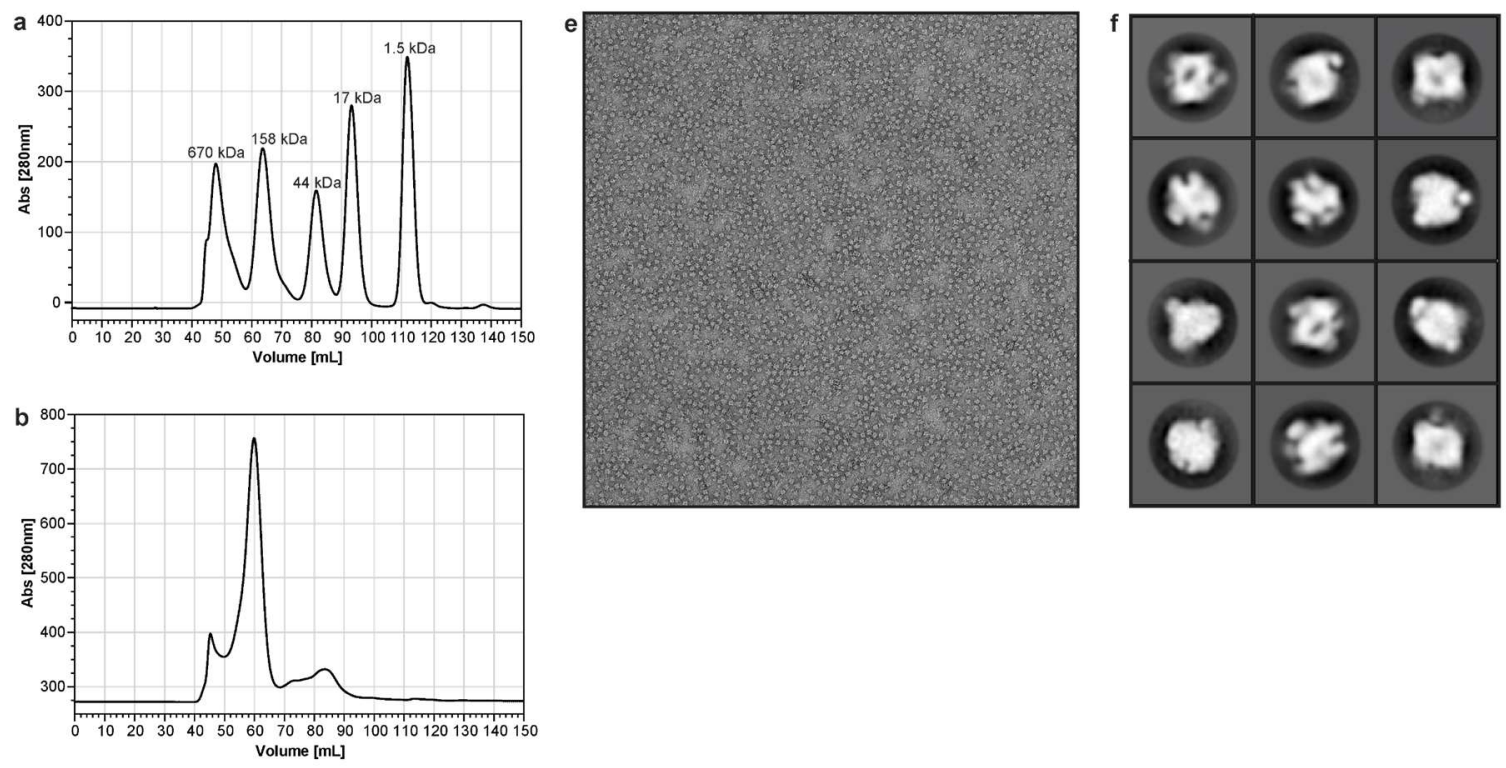

1481

1482

1483

1484

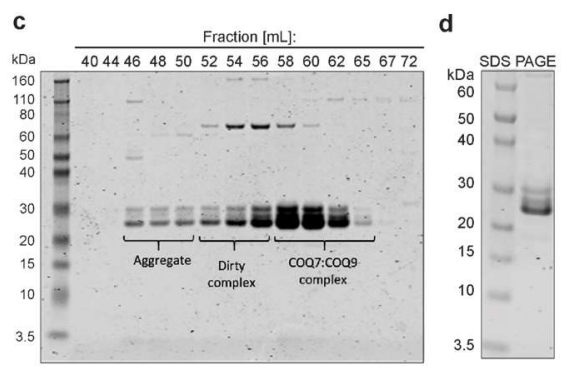

1485 
1494 Extended Data Figure 1. Preliminary biochemical and structural analysis of the

1495 COQ7:COQ9 complex.

1496 a, Size exclusion chromatography trace of protein mass standards. b, Size exclusion 1497 chromatography trace of the purified GB1- ${ }^{\mathrm{Nd} 38} \mathrm{COQ7}:$ His6- ${ }^{\mathrm{Nd} 79} \mathrm{COQ} 9$ complex $\mathbf{c}$, SDS-PAGE 1498 analysis of collected fractions. d, SDS-PAGE analysis of pooled GB1- ${ }^{\mathrm{Nd} 38} \mathrm{COQ} 7: \mathrm{His} 6-{ }^{\mathrm{Nd} 79} \mathrm{COQ9}$

1499 fractions submitted to cryoEM analysis. e, Representative negative-stain EM image of 1500 COQ7:COQ9 complex reveals mono-disperse particle distribution with very little protein 1501 aggregation. f, Representative 2D class averages of COQ7:COQ9 complex from the negative-stain 1502 data collected on Tecnai T12 microscope equipped with CCD camera showing that particles have 1503 well-defined shapes and symmetric features.

1504

1505

1506

1507

1508

1509

1510

1511

1512

1513

1514

1515

1516 
bioRxiv preprint doi: https://doi.org/10.1101/2021.11.15.468694; this version posted November 15,2021 . The copyright holder for this preprint (which was not certified by peer review) is the author/funder, who has granted bioRxiv a license to display the preprint in perpetuity. It is made available under aCC-BY-NC-ND 4.0 International license.

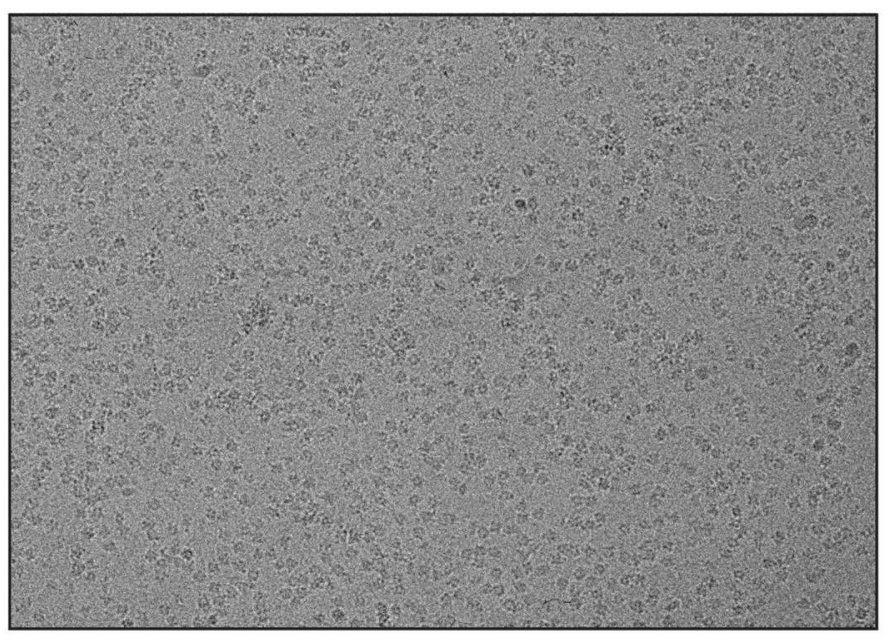

b

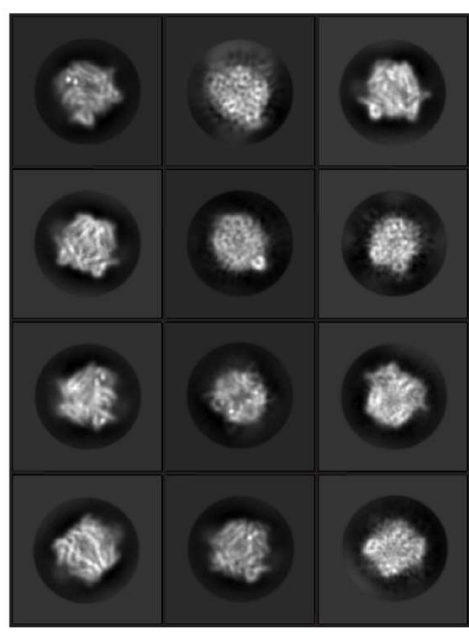

1525

1526

1527

1528
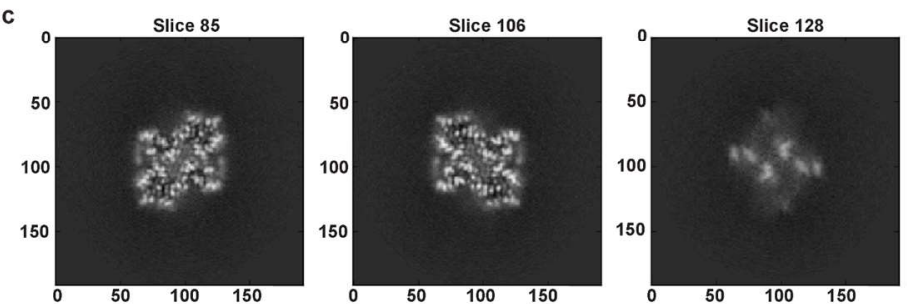

1529

1530

1531

1532
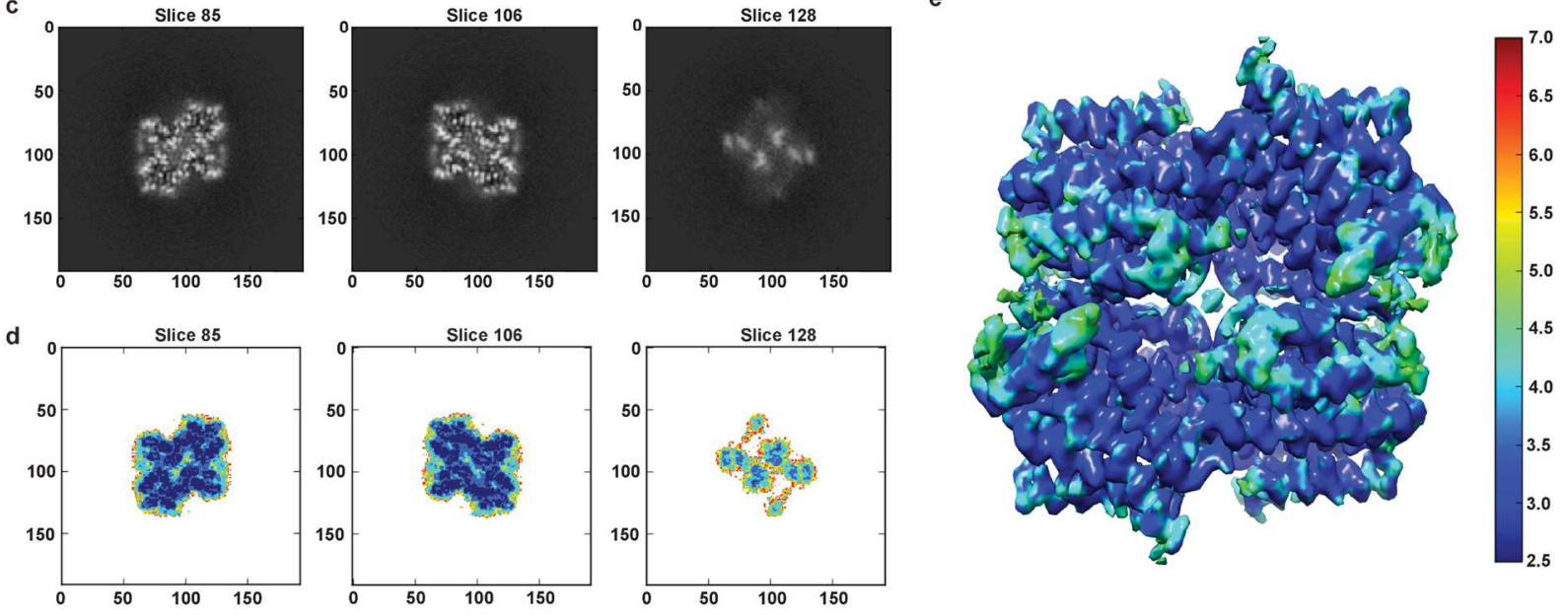

1533

1534

1535

1536

1537

1538

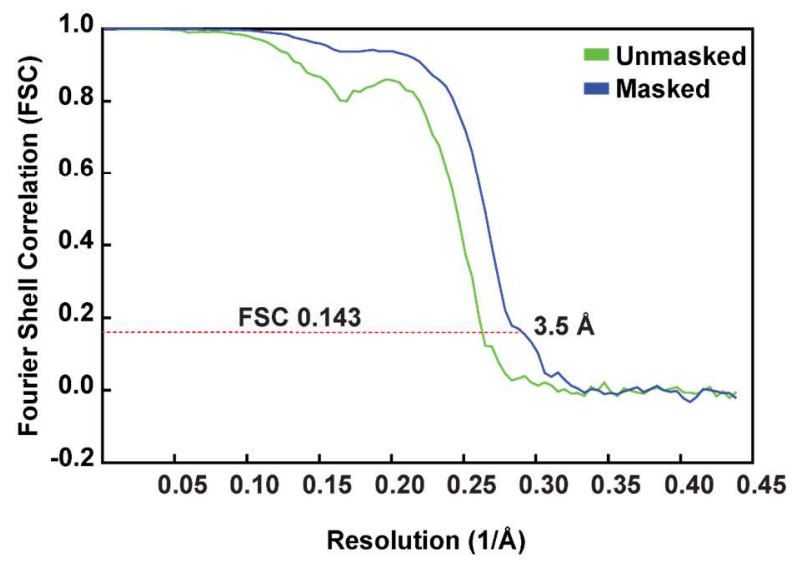

g

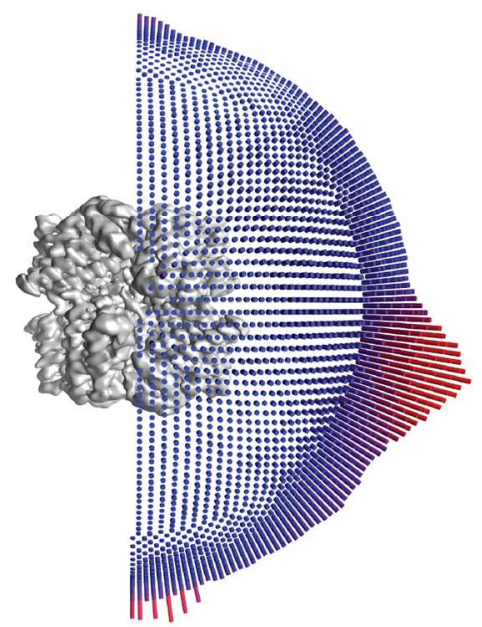

1539 
1540 Extended Data Figure 2. Single-particle cryoEM of unliganded COQ7:COQ9 complex.

1541 a, Representative motion-corrected electron micrograph of COQ7:COQ9 complex only. b, Gallery

1542 of 2D class averages calculated from the cryoEM data of the complex. c, d, Slices through the

1543 unsharpened density map at distinct levels are shown in top view. e, Final 3D electron density map

1544 colored according to local resolution is shown in side view. Local resolution was calculated by

1545 ResMap. f, Fourier shell coefficient (FSC) curves (cutoff of 0.143) between two independently

1546 refined half maps before and after post-processing. g, Orientation distribution of all particle images

1547 included in the calculation of the final 3D reconstruction.

1548

1549

1550

1551

1552

1553

1554

1555

1556

1557

1558

1559

1560

1561 
bioRxiv preprint doi: https://doi.org/10.1101/2021.11.15.468694; this version posted November 15, 2021. The copyright holder for this preprint (which was not certified by peer review) is the author/funder, who has granted bioRxiv a license to display the preprint in perpetuity. It is made available under aCC-BY-NC-ND 4.0 International license.



$20.9 \%$

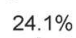

$26.0 \%$

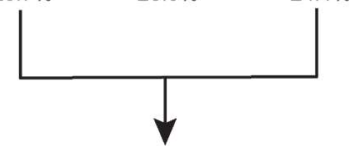

$$
\begin{gathered}
\begin{array}{c}
\text { 3D Refinement } \\
\text { (281,866 particles) })
\end{array} \\
\text { D2 symmetry } \downarrow \text { Ctf Refinement }
\end{gathered}
$$

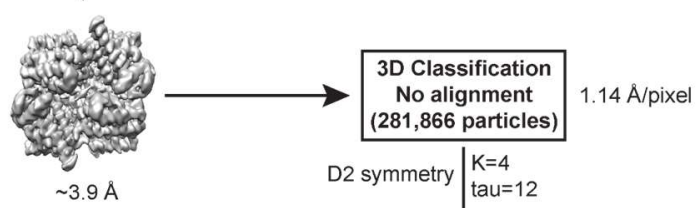

1581

1582

1583

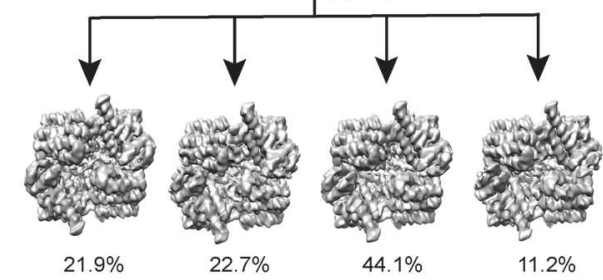

$1.9 \%$

$44.1 \%$

$11.2 \%$

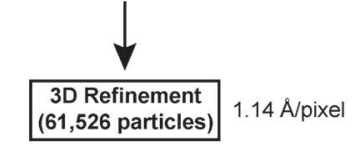

D2 symmetry Ctf Refinement

1584

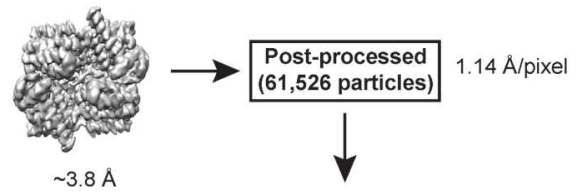

1585 
1586 Extended Data Figure 3. Stages of preprocessing, image classification, refinement, and

1587 postprocessing for unliganded COQ7:COQ9 complex. Details can be found in the image

1588 analysis and 3D reconstruction section of the Methods.

1589

1590

1591

1592

1593

1594

1595

1596

1597

1598

1599

1600

1601

1602

1603

1604

1605

1606

1607

1608 
bioRxiv preprint doi: $\mathrm{https}$ //doi.org/10.1101/2021.11.15.468694; this version posted November 15,2021 . The copyright holder for this preprint (which was not certified by peer review) is the author/funder, who has granted bioRxiv a license to display the preprint in perpetuity. It is made available under aCC-BY-NC-ND 4.0 International license.

1610

1611

1612

1613

1614

1615

1616

1617

1618

1619

1620

1621

1622

1623

1624

1625

1626

1627

1628

1629

1630

1631

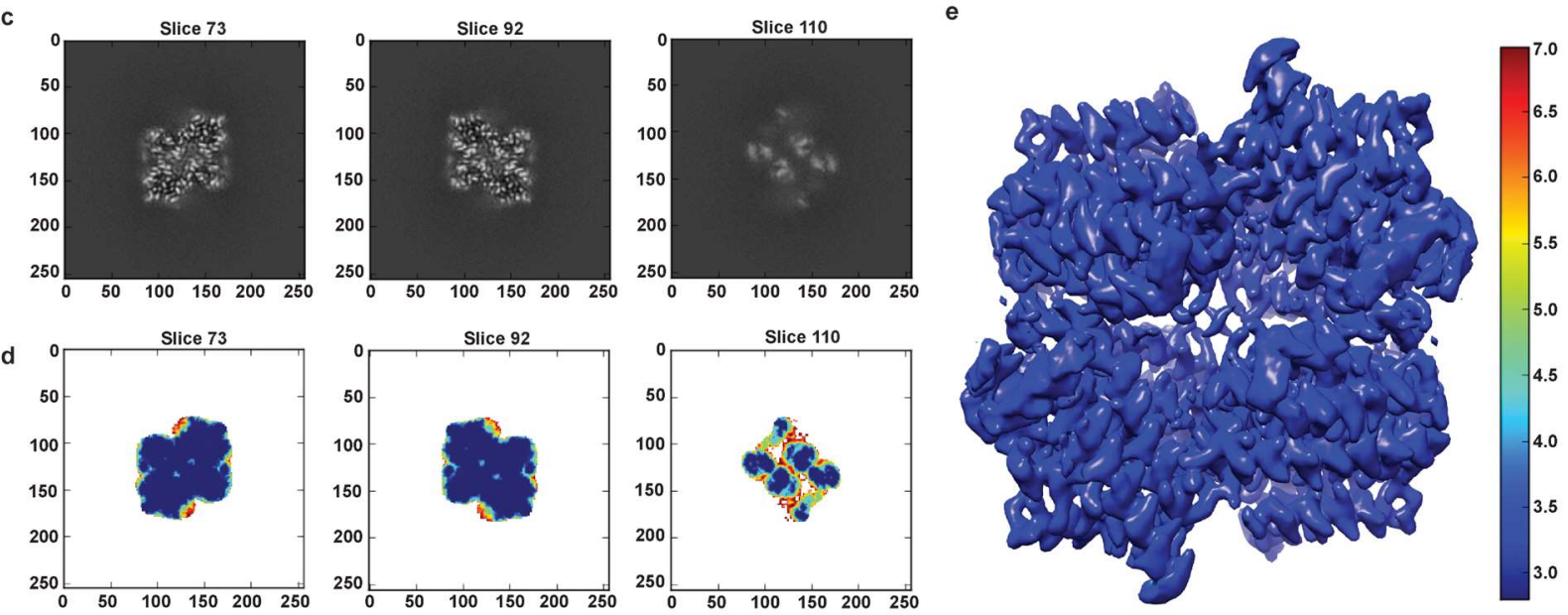
1632 Extended Data Figure 4. Single-particle cryoEM of NADH-bound COQ7:COQ9 complex.

1633 a, Representative motion-corrected electron micrograph of NADH-bound COQ7:COQ9 complex.

1634 b, Representative 2D class averages calculated from the cryoEM data of the complex. c,d, Slices

1635 through the unsharpened density map at distinct levels are shown in top view. e, Final 3D electron

1636 density map colored according to local resolution is shown in side view. Local resolution was

1637 calculated by ResMap. f, Fourier shell coefficient (FSC) curves (cutoff of 0.143) between two

1638 independently refined half maps before and after post-processing. g, Orientation distribution of all

1639 particle images included in the calculation of the final 3D reconstruction.

1640

1641

1642

1643

1644

1645

1646

1647

1648

1649

1650

1651

1652

1653

1654 
bioRxiv preprint doi: https://doi.org/10.1101/2021.11.15.468694; this version posted November 15, 2021. The copyright holder for this preprint (which was not certified by peer review) is the author/funder, who has granted bioRxiv a license to display the preprint in perpetuity. It is made available under aCC-BY-NC-ND 4.0 International license.

\section{Extended Data Figure 5}

1656

1657

1658

1659

1660

1661

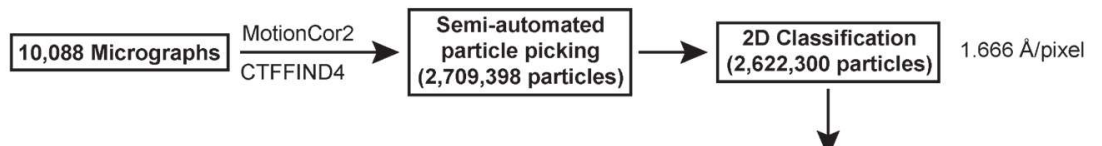

1.666 Å/pixel

$(2,622,300$ particles $)$

\begin{tabular}{l|l} 
C1 symmetry & $\begin{array}{l}\mathrm{K}=6 \\
\text { tau=12 }\end{array}$
\end{tabular}

1662

1663

1664

1665

1666

1667

1668



$22.0 \%$

$19.4 \%$

$24.7 \%$

Re-extract



3D Refinement
$(1,249,119$ particles

$0.833 \AA /$ pixel

D2 symmetry Ctf Refinement

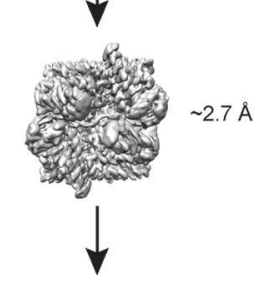

\section{D Classification \\ No alignment}

$0.833 \AA / p i x e l$

1669

1670

1671

1672

D2 symmetry $K=$

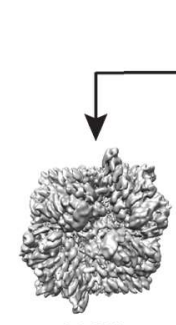

$23.8 \%$

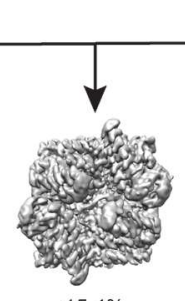

$15.4 \%$

$13.3 \%$

tau $=16$

1673

1674

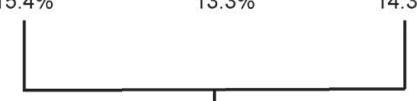

$4.3 \%$

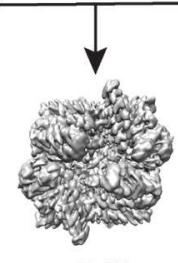

$17.1 \%$

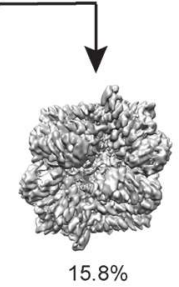

1676

1677

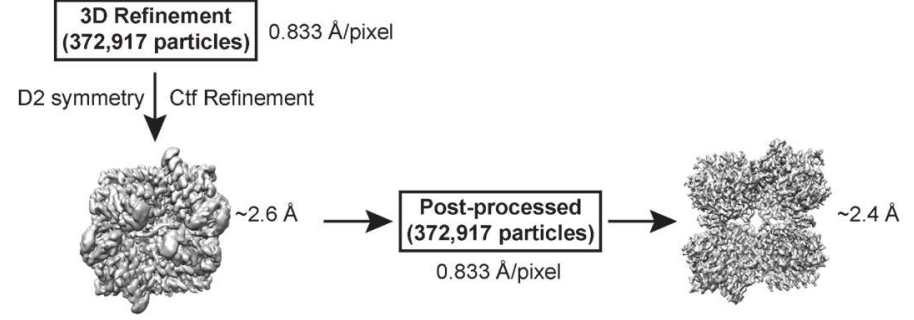


bioRxiv preprint doi: https://doi org/10.1101/2021.11.15.468694; this version posted November 15, 2021. The copyright holder for this preprint

(which was not certified by peer review) is the author/funder, who has granted bioRxiv a license to display the preprint in perpetuity. It is made available under aCC-BY-NC-ND 4.0 International license.

1678 Extended Data Figure 5. Flowchart for cryoEM data processing of NADH-bound

1679 COQ7:COQ9 complex. Details can be found in the image analysis and 3D reconstruction section

1680 of the Methods.

1681

1682

1683

1684

1685

1686

1687

1688

1689

1690

1691

1692

1693

1694

1695

1696

1697

1698

1699

1700 
1704

1705
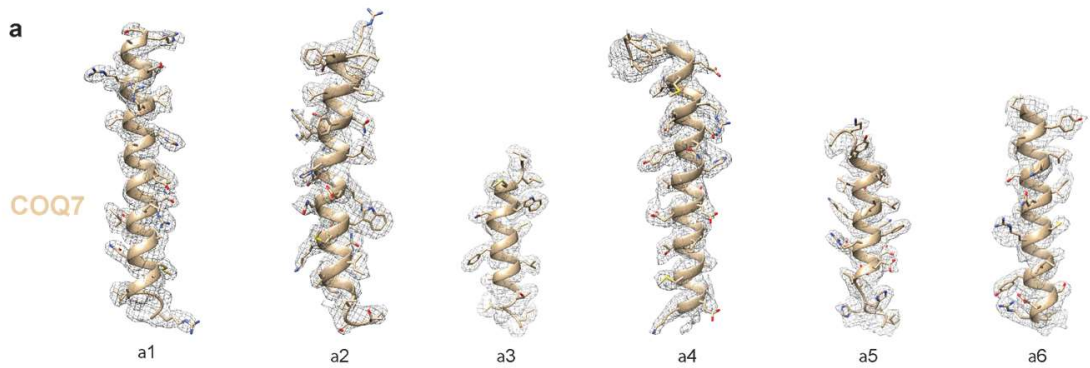

$1706 \quad b$

1707

1708

1709

b

a1
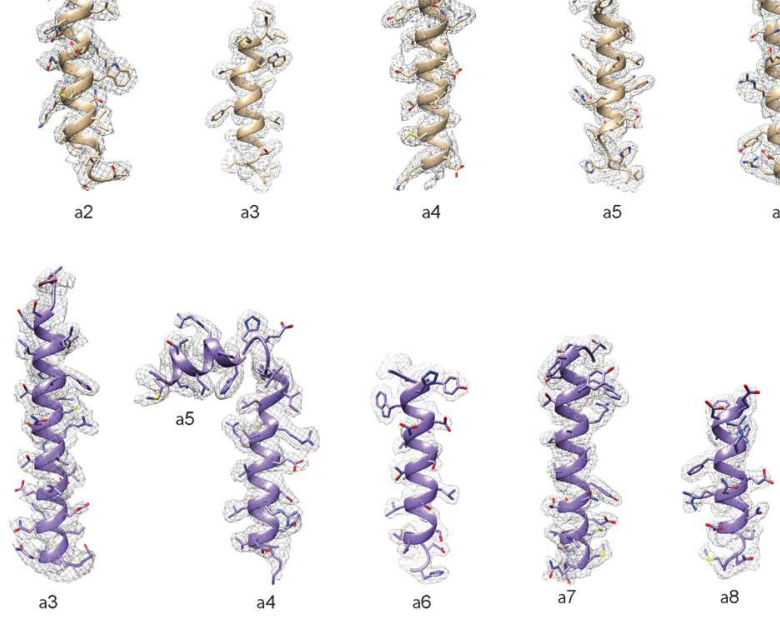

1710

1711

c

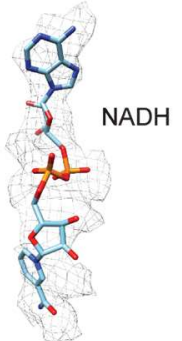

d

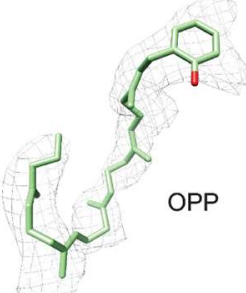

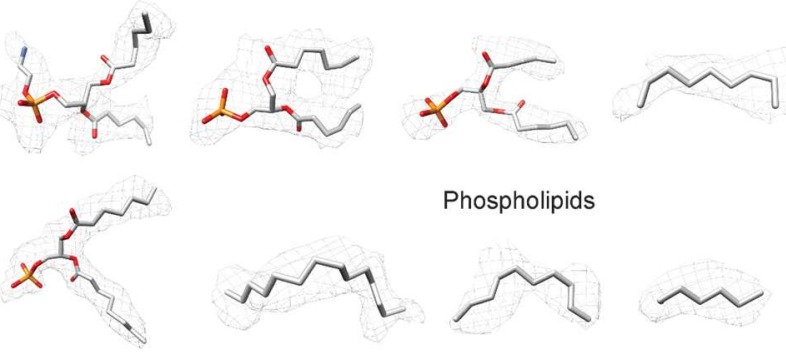

1714

1715

1716

1717

1718

1719

1720

1721

1722

1723
Extended Data Figure 6. Snapshots of map versus model agreement by secondary

structure. Refined atomic models fit well into corresponding densities. a,b, Representative densities include the $\alpha$-helical regions of COQ7 (beige) and COQ9 (purple) are shown in the context of the atomic model with side chains are shown as sticks and the backbone as ribbons. $\mathbf{c}, \mathbf{d}, \mathbf{e}$, Representative regions of density from the electron density map for NADH (cyan), OPP (green), and phospholipid (gray) molecules are presented at the bottom panel. 
bioRxiv preprint doi: https://doi.org/10.1101/2021.11.15.468694 - this version posted November 15, 2021. The copyright holder for this preprint (which was not certified by peer review) is the author/funder, who has granted bioRxiv a license to display the preprint in perpetuity. It is made available under aCC-BY-NC-ND 4.0 International license.

1724 Extended Data Figure 7

1725

1726

1727

1728

1729

1730

1731

1732

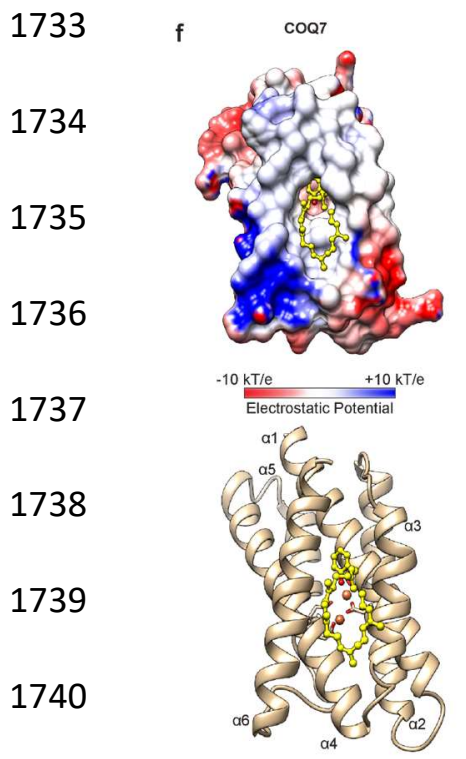

b



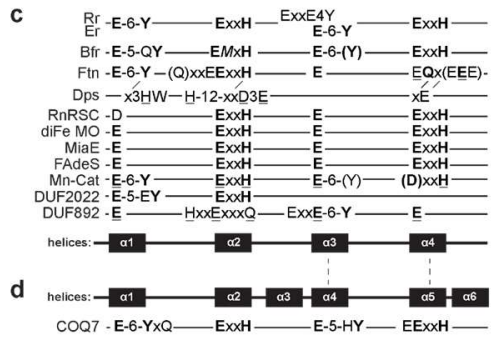

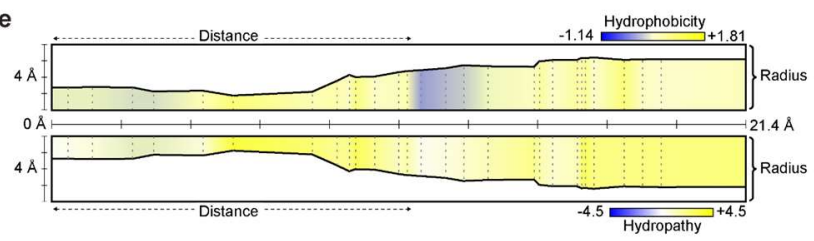

1741



g



h

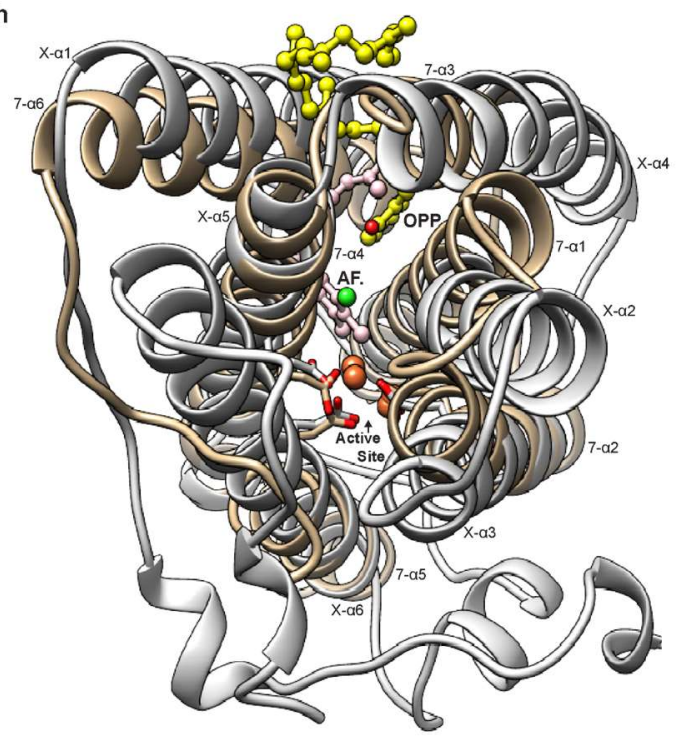

1742

1743

1744

1745

1746 
1747 Extended Data Figure 7. Comparison of COQ7 to bactoferritin and alternative oxidase.

1748 a, Structural comparison of cryoEM COQ7 (tan) to bactoferritin monomer (gray) PDB:4AM2 b,

1749 Structural features known to be important for oligomerization and function of ferritins but

1750 mutated in COQ7 are shown in green. c, Conservation of the (E-(X6)-Y-(X 22$)-\mathrm{E}-\left(\mathrm{X}_{2}\right)-\mathrm{H}-\left(\mathrm{X}_{48}\right)-\mathrm{E}-$

$\left.1751\left(\mathrm{X}_{6}\right)-\mathrm{Y}-\left(\mathrm{X}_{28}\right)-\mathrm{E}-\left(\mathrm{X}_{2}\right)-\mathrm{H}\right)$ motif among diiron proteins. $\mathbf{d}$, topology of COQ7 in comparison to

1752 other diiron proteins. e, Properties of the hydrophobic channel leading to the COQ7's active site

1753 calculated by MOLEonline. Panels $\mathbf{c}$ and $\mathbf{d}$ were reproduced with changes from Andrews, S.C $\mathrm{C}^{27}$.

1754 f, Surface and ribbon view of COQ7. g, Surface and ribbon view of alternative oxidase

1755 PDB:3VVA (AOX). h, Structural comparison of COQ7 and AOX. Bound aromatic

1756 Ascofuranone-derivative inhibitor (pink, AF.) and resolved diiron active site (red spheres)

1757 compared to COQ7 (tan) with bound OPP (yellow). Helices of COQ7 (7- $\alpha$ ) and AOX (X- $\alpha)$ are

1758 numbered.

1759

1760

1761

1762

1763

1764

1765

1766

1767

1768

1769 
bioRxiv preprint doi: https://doi.org/10.1101/2021.11.15.468694; this version posted November 15,2021 . The copyright holder for this preprint (which was not certified by peer review) is the author/funder, who has granted bioRxiv a license to display the preprint in perpetuity. It is made available under aCC-BY-NC-ND 4.0 International license.

1770 Extended Data Figure 8

1771

1772

1773

1774

a
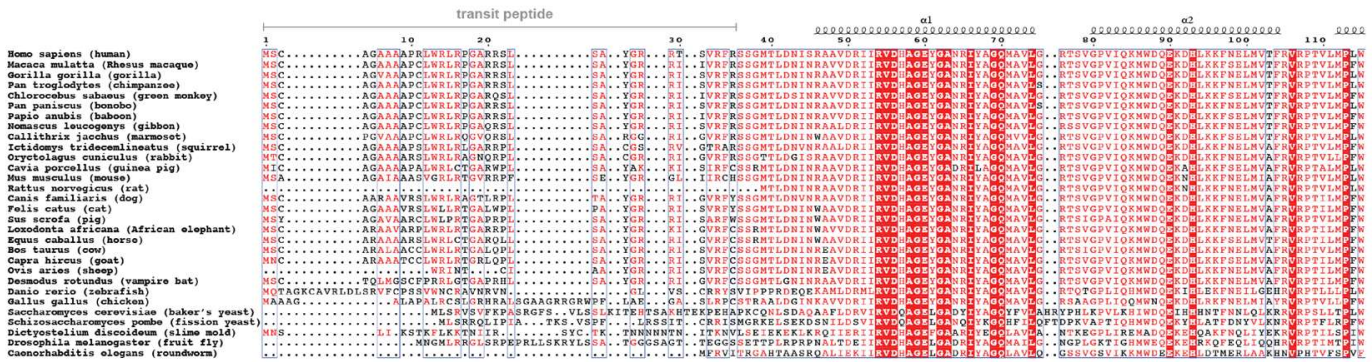

1775

1776

1777

1778
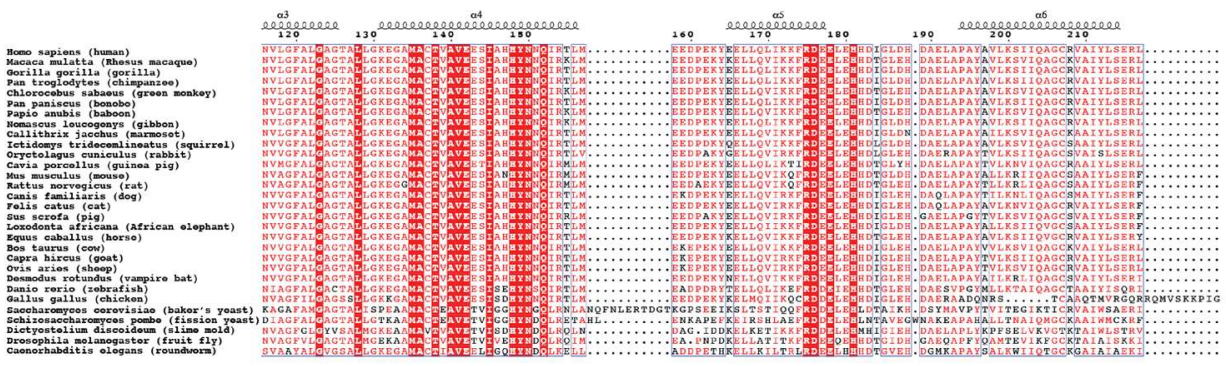

1779 b

1780

1781

1782

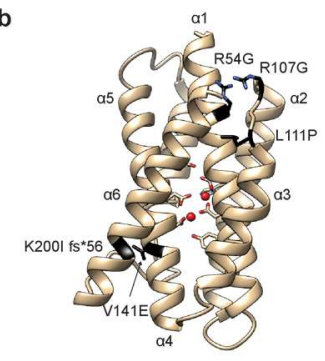

1783

1784

1785

1786

1787

1788

1789

1790

1791 

in COQ7. sequences from Homo sapiens (human; Uniprot: Q99807), Macaca mulatta (rhesus macaque; Uniprot: A0A5F7ZCW4), Gorilla gorilla (gorilla; Uniprot: G3QJN6), Pan troglodytes

1797 (Chimpanzee; Uniprot: H2QAN4), Chlorocebus sabaeus (green monkey; Uniprot: A0A0D9R654), Nomascus leucogenys (gibbon; Uniprot: A0A2I3H0N0), Callithrix jacchus (marmoset; Uniprot: F6XXM7), Ictidomys tridecemlineatus (squirrel; Uniprot: I3M379), Oryctolagus cuniculus (rabbit; Uniprot: G1SGS8), Cavia porcellus (guinea pig; Uniprot: H0VHI5), Mus musculus (mouse; Uniprot: P97478), Rattus norvegicus (rat; Uniprot: Q63619), Canis familiaris (dog, Uniprot: E2RF61), Felis catus (cat; Uniprot: M3WJK9), Sus scrofa (pig; Uniprot: A0A287A0B5), Loxodonta africana (African elephant; Uniprot: G3STP5), Equus caballus (horse; Uniprot: F6YA13), Bos taurus (cow; Uniprot: Q2TBW2), Capra hircus (goat; Uniprot: A0A452EGI8), Ovis aries (sheep; Uniprot: W5PPJ7), Desmodus rotundus (Vampire bat; Uniprot: K9IHU0), Danio rerio (zebrafish; Uniprot: F1QW05), Gallus gallus (chicken; Uniprot: F1NIP2), Saccharomyces cerevisiae (baker's yeast; Uniprot: P41735), Schizosaccharomyces pombe (fission yeast; Uniprot:

1809 O74826), Dictyostelium discoideum (slime mold; Uniprot: Q54VB3), Drosophila melanogaster 1810 (fruit fly; Uniprot: Q9W3W4), and Caenorhabditis elegans (roundworm; Uniprot: P48376) are 1811 aligned. Complete conservation of a given amino acid is indicated with red boxes. Secondary 1812 structural elements observed in the cryoEM structure of COQ7 are shown as coils for $\alpha$-helices. 1813 b, Structure of COQ7 with detrimental mutations identified in patients based on ClinVar. 
bioRxiv preprint doi: https://doi.org/10.1101/2021.11.15.468694; this version posted November 15,2021 . The copyright holder for this preprint (which was not certified by peer review) is the author/funder, who has granted bioRxiv a license to display the preprint in perpetuity. It is made available under aCC-BY-NC-ND 4.0 International license.

1815

1816

1817

1818

1819

1820

1821

1822

1823

1824

1825

1826

1827

1828

1829

1830

1831

1832

1833

1834

1835
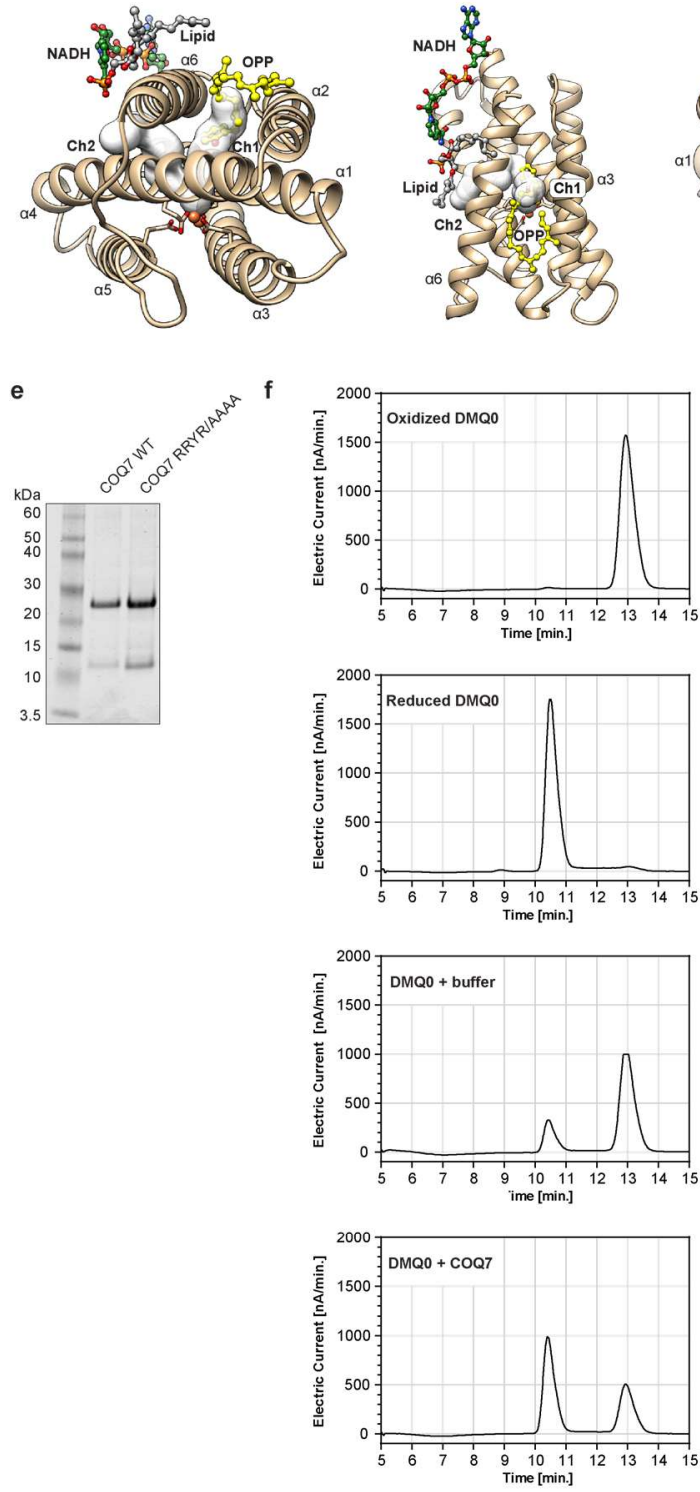
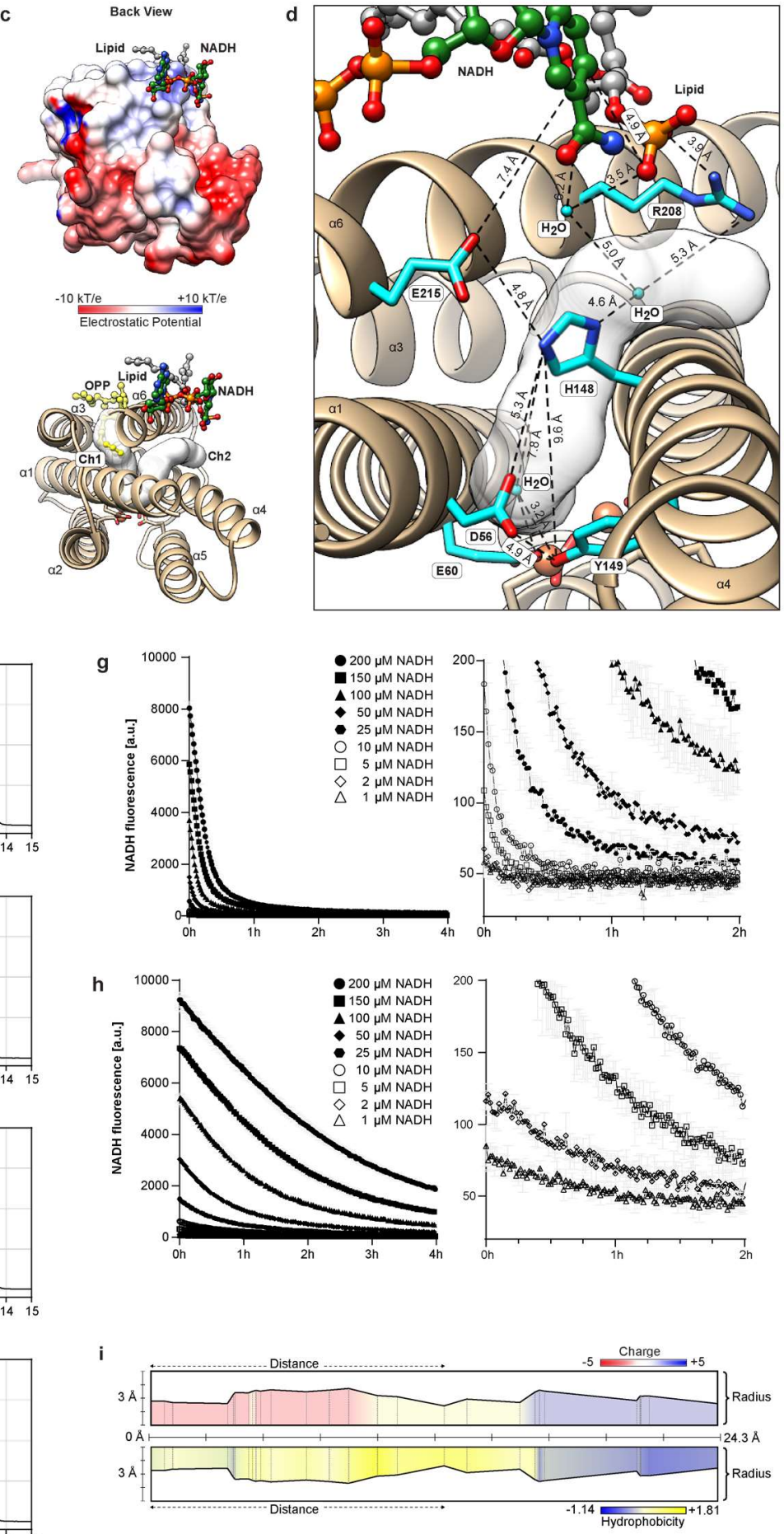


\section{Extended Data Figure 9. Additional details of NADH binding and utilization by COQ7.}

$\mathbf{a}, \mathbf{b}, \mathbf{c}, \mathbf{d}$, Additional poses presenting NADH binding site on COQ7 and channels leading to the active site. The OPP binding channel is labeled ch1 and the hypothesized charge transfer channel is labeled ch2. e, SDS-PAGE gel showing digitonin purified His6-GB1 - ${ }^{\mathrm{N} 3}{ }^{8} \mathrm{COQ} 7 \mathrm{WT}$ and His6GB1- ${ }^{\mathrm{Nd} 38} \mathrm{COQ} 7{ }^{\mathrm{R} 51 \mathrm{~A} / \mathrm{R} 208 \mathrm{~A} / \mathrm{Y} 212 \mathrm{~A} / \mathrm{R} 216 \mathrm{~A}}$ mutants. f, HPLC-ECD analysis showing COQ7-dependent

1843 reduction of the quinone substrate $\mathrm{DMQ}_{0}$ by NADH present in the buffer. g, h, In vitro NADH 1844 titration experiments with COQ7 WT and COQ7 RRYR/AAAA (mean \pm s.d., $n=3$ ). Panels on

1845 the right are zooms in of the panels on the left. i, Properties of the channel ch2 calculated by MOLEonline. 
bioRxiv preprint doi: https://doi.org/10.1101/2021.11.15.468694; this version posted November 15,2021 . The copyright holder for this preprint (which was not certified by peer review) is the author/funder, who has granted bioRxiv a license to display the preprint in perpetuity. It is made available under aCC-BY-NC-ND 4.0 International license.

1861

1862

1863

1864

1865

1866

1867

1868

1869

1870

1871

1872

1873

1874

1875

1876

1877

c
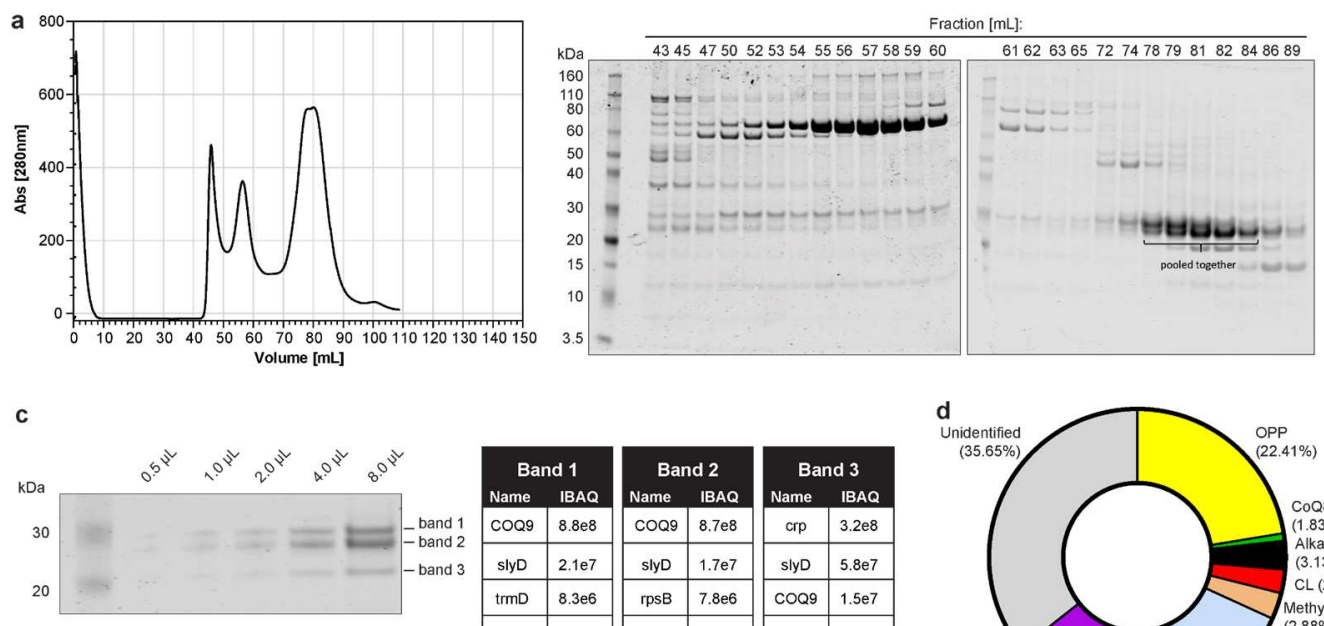

\begin{tabular}{|c|c|c|c|c|c|}
\hline \multicolumn{2}{|c|}{ Band 1} & \multicolumn{2}{|c|}{ Band 2} & \multicolumn{2}{|c|}{ Band 3} \\
\hline Name & IBAQ & Name & IBAQ & Name & IBAC \\
\hline COQ9 & $8.8 \mathrm{e} 8$ & COQ9 & $8.7 \mathrm{e} 8$ & crp & $3.2 \mathrm{e} 8$ \\
\hline slyD & 2.1e7 & slyD & $1.7 \mathrm{e} 7$ & slyD & $5.8 \mathrm{e} 7$ \\
\hline trmD & $8.3 \mathrm{e} 6$ & $\mathrm{rpsB}$ & $7.8 \mathrm{e} 6$ & COQ9 & $1.5 \mathrm{e} 7$ \\
\hline $\mathrm{crp}$ & 3.1e6 & crp & $6.8 \mathrm{e} 6$ & folE & $6.8 \mathrm{e} 6$ \\
\hline rpsB & $1.1 \mathrm{e} 6$ & gpmA & $1.8 \mathrm{e} 6$ & gntK & $5.1 \mathrm{e} 6$ \\
\hline
\end{tabular}

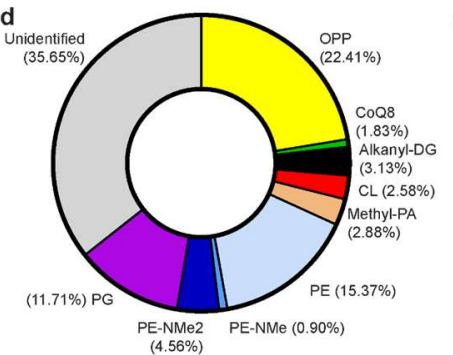

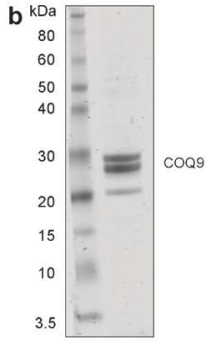

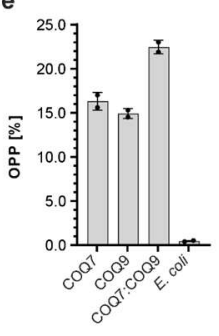

1878

1879

1880

1881

1882

1883 
1884 Extended Data Figure 10. Analysis of COQ7:COQ9 dimer and tetramer.

1885 a, Size exclusion chromatography trace of His6- ${ }^{\mathrm{Nd} 79} \mathrm{COQ} 9^{\mathrm{W} 240 \mathrm{~K}}$ co-expressed with GB1-

$1886{ }^{\mathrm{Nd} 38} \mathrm{COQ7}$. b, SDS-PAGE gel showing composition of the pooled fractions. c, LC-MS/MS

1887 identification of proteins in the bands. No COQ7 was detected. d, Lipids detected by LC-MS in

1888 the purified GB1- ${ }^{\mathrm{Nd} 38} \mathrm{COQ7}$ :His6- ${ }^{\mathrm{Nd} 79} \mathrm{COQ} 9$ complex. e, LC-MS measured level of OPP in

1889 individually purified His6-GB1- ${ }^{\mathrm{Nd} 38} \mathrm{COQ7}$, His6- ${ }^{\mathrm{Nd} 79} \mathrm{COQ9}$, GB1- ${ }^{\mathrm{Nd} 38} \mathrm{COQ7}$ :His6- ${ }^{\mathrm{Nd} 79} \mathrm{COQ9}$ or

1890 E.coli cells. Data expressed as \% of total lipid signal of each sample (mean \pm s.d., $n=2)$.

1891

1892

1893

1894

1895

1896

1897

1898

1899

1900

1901

1902

1903

1904

1905

1906 
bioRxiv preprint doi: $h t t p s: / / d o i . o r g / 10.1101 / 2021.11 .15 .468694$; this version posted November 15,2021 . The copyright holder for this preprint

(which was not certified by peer review) is the author/funder, who has granted bioRxiv a license to display the preprint in perpetuity. It is made available under aCC-BY-NC-ND 4.0 International license.
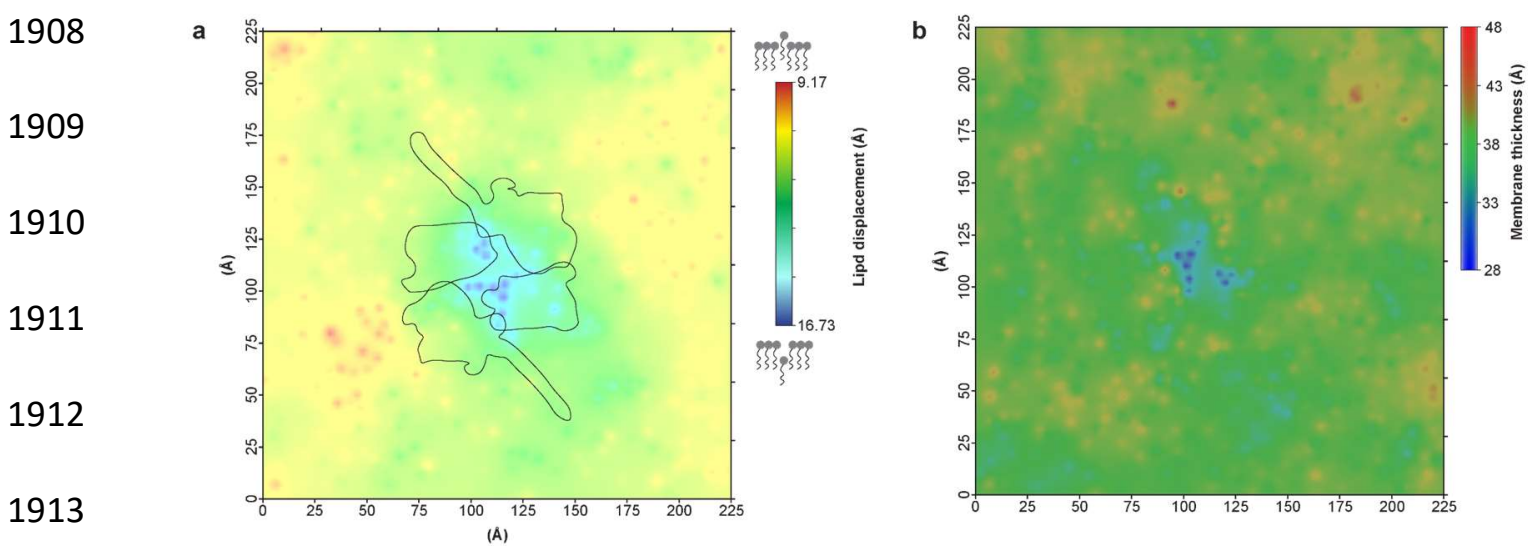

1914

1915

1916

1917

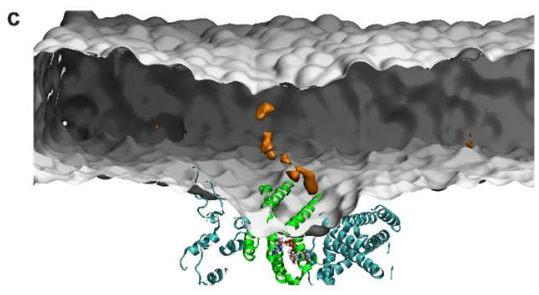

1918

1919

1920
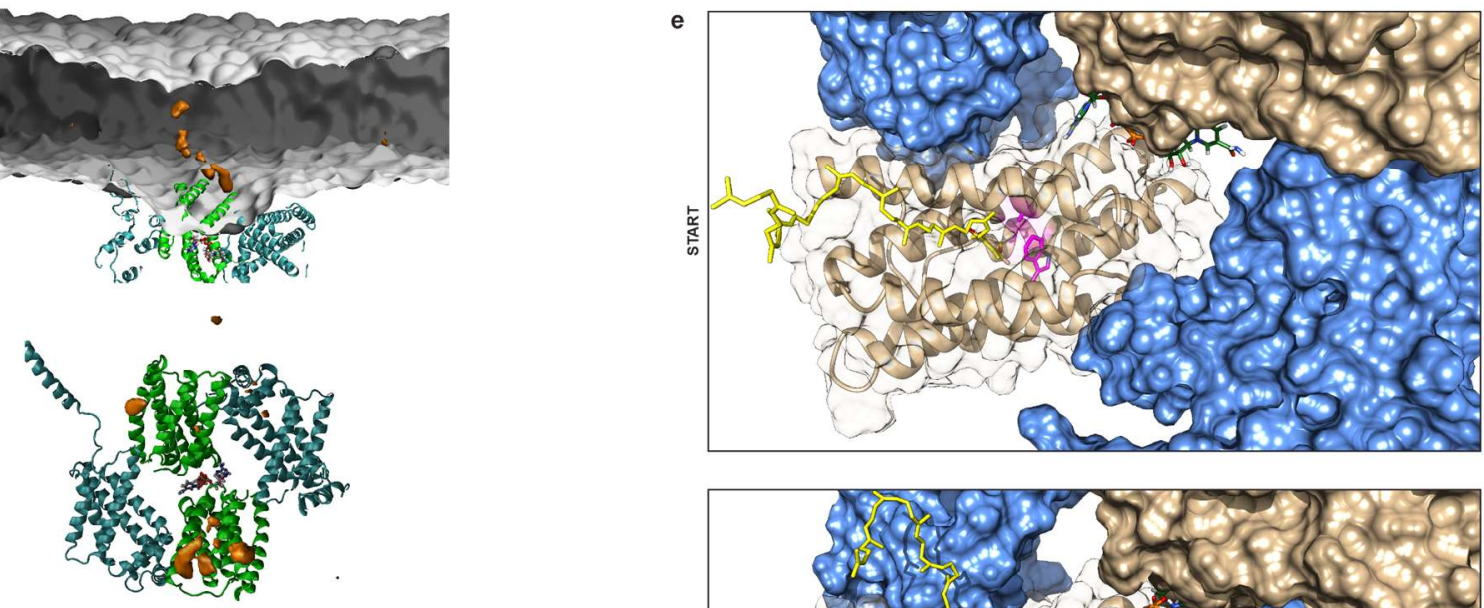

1921

1922

d



1925

1926

1927

1928
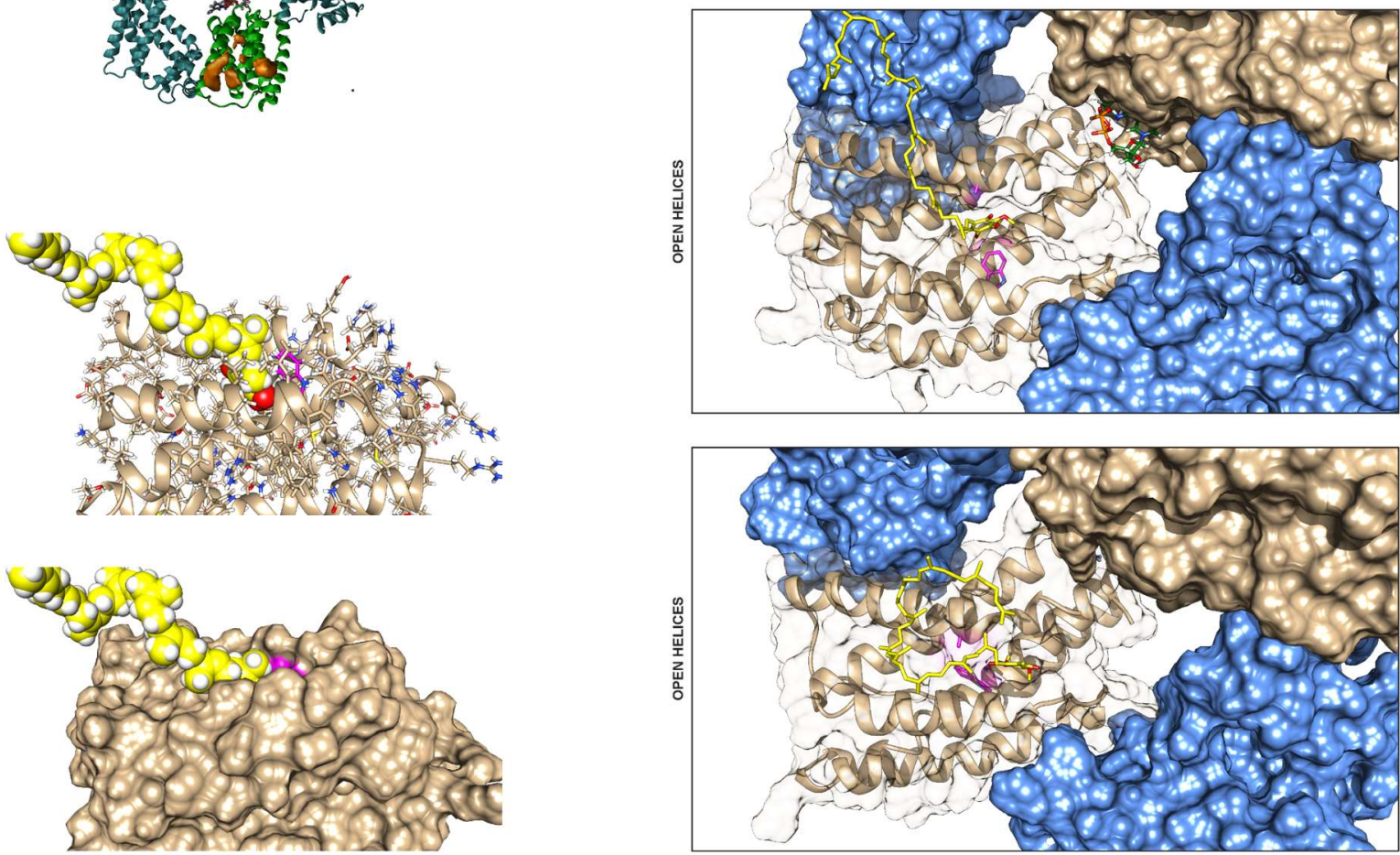
1930 Extended Data Figure 11. Additional data from COQ7:COQ9 MD simulation.

1931 a, Map of the deformation of the top membrane leaflet induced by the COQ7:COQ9 tetramer. b,

1932 Map of the changes of membrane thickness induced by the COQ7:COQ9 tetramer.

1933 c, Time-averaged density of CoQ10 molecules represented as brown volume/surface. d, Two

1934 views of a CoQ10 molecule modeled into COQ7 based on the OPP observed in the CryoEM

1935 structure. e, Additional representation of COQ7 conformational changes and CoQ10 movement

1936 visible in Fig. 5d.

1937

1938

1939

1940

1941

1942

1943

1944

1945

1946

1947

1948

1949

1950

1951

1952

1953 
Extended Data Table 1. CryoEM data collection, refinement, and validation statistics.

\begin{tabular}{|c|c|c|}
\hline & $\begin{array}{c}\text { NADH-bound COQ7:COQ9 } \\
\text { complex } \\
\text { (EMD-25413) } \\
\text { (PDB 7SSS) }\end{array}$ & $\begin{array}{c}\text { COQ7:COQ9 complex only } \\
\text { (EMD-25412) } \\
\text { (PDB 7SSP) }\end{array}$ \\
\hline \multicolumn{3}{|l|}{ Data collection and processing } \\
\hline Microscope & FEI Titan Krios & FEI Talos Arctica \\
\hline Camera & Gatan K3 Summit & Gatan K3 Summit \\
\hline Magnification & $105,000 x$ & $36,000 x$ \\
\hline Voltage (kV) & 300 & 200 \\
\hline Electron exposure $\left(\mathrm{e}^{-} / \AA^{2}\right)$ & 65 & 58.8 \\
\hline Defocus $(\mu \mathrm{m})$ & -0.3 to -1.2 & -0.5 to -1.5 \\
\hline Pixel size $(\AA)$ & 0.833 & 1.14 \\
\hline Symmetry imposed & D2 & D2 \\
\hline Micrographs (no.) & 10,088 & 1,395 \\
\hline Initial particle images (no.) & $2,709,398$ & 574,760 \\
\hline Final particle images (no.) & 372,917 & 61,526 \\
\hline Map resolution $(\AA)$ & 2.4 & 3.5 \\
\hline FSC threshold & 0.143 & 0.143 \\
\hline Map resolution range $(\AA)$ & 2.2 to 6.7 & 3.4 to 5.9 \\
\hline \multicolumn{3}{|l|}{ Refinement } \\
\hline Initial model used (PDB code) & COQ7 (de novo) - COQ9 (6AWL) & COQ7 (de novo) - COQ9 (6AWL) \\
\hline Model resolution $(\AA)$ & 2.4 & 3.5 \\
\hline FSC threshold & 0.143 & 0.143 \\
\hline Map sharpening $B$ factor $\left(\AA^{2}\right)$ & -95.99 & -156.07 \\
\hline \multicolumn{3}{|l|}{ Model composition } \\
\hline Nonhydrogen atoms & 12,190 & 10,930 \\
\hline Protein residues & 1,416 & 1,376 \\
\hline Ligands & 4 & \\
\hline Lipids & 32 & \\
\hline Cofactor & 2 & \\
\hline Water & 170 & \\
\hline \multicolumn{3}{|l|}{ B factors $\left(\AA^{2}\right)$} \\
\hline Protein & 25.11 & 22.16 \\
\hline Ligands & 43.18 & \\
\hline Water & 30.84 & \\
\hline \multicolumn{3}{|l|}{ R.m.s. deviations } \\
\hline Bond lengths $(\AA)$ & 0.004 & 0.004 \\
\hline Bond angles $(\AA)$ & 0.449 & 0.611 \\
\hline \multicolumn{3}{|l|}{ Validation } \\
\hline MolProbity score & 1.27 & 1.67 \\
\hline Clashscore & 4.01 & 4.52 \\
\hline Poor rotamers (\%) & 0.0 & 0.0 \\
\hline \multicolumn{3}{|l|}{ Ramachandran plot } \\
\hline Favored (\%) & 97.63 & 93.15 \\
\hline Allowed (\%) & 2.37 & 6.85 \\
\hline Disallowed (\%) & 0.0 & 0.0 \\
\hline
\end{tabular}

